\title{
Catalytic Formal Homo-Nazarov Cyclization
}

\author{
Filippo De Simone, Julien Andrès, Riccardo Torosantucci and \\ Jérôme Waser*
}

Laboratory of Catalysis and Organic Synthesis

Ecole Polytechnique Fédérale de Lausanne

EPFL SB ISIC LCSO, BCH 4306, 1015 Lausanne, Switzerland

jerome.waser@epfl.ch

Received Date (will be automatically inserted after manuscript is accepted)

\section{ABSTRACT}

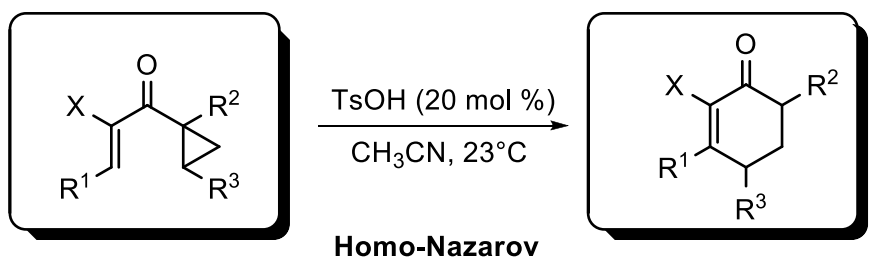

The first catalytic method for the cyclization of vinyl-cyclopropyl ketones (formal homo-Nazarov reaction) is reported. Starting from activated cyclopropanes, heterocyclic and carbocyclic compounds were obtained under mild conditions using Brønsted acid catalysts. Preliminary investigation of the reaction mechanism indicated a stepwise process.

Carbocyclic and heterocyclic scaffolds occupy a privileged position in both natural products and pharmaceuticals. ${ }^{1}$ Consequently, the development of cyclization and cycloaddition reactions for the efficient formation of cyclic structures is a very important goal in organic chemistry. In this respect, the development of new, highly stereoselective catalytic methods is crucial to allow a more efficient and environmentally friendly access to polycyclic molecules. ${ }^{2}$

One classical approach towards the construction of cyclopentenone rings is the Nazarov reaction, which is the electrocyclic ring closure of a pentadienyl cation, followed by proton transfer $(\mathbf{A}$, Scheme 1$){ }^{3}$ The potential

(1) Clardy, J.; Walsh C. Nature 2004, 432, 829.

(2) (a) Balskus, E. P.; Jacobsen, E. N. Science 2007, 317, 1736. (b) Mohr, J. T.; Krout, M. R.; Stoltz, B. M. Nature 2008, 455, 323.

(3) (a) Nazarov, I. N.; Zaretskaya, I. I. Izv. Akad. Nauk. SSSR. Ser. Khim. 1941, 211. (b) Habermas, K. L.; Denmark, S. E.; Jones, T. K.; Org. React. (N. Y.) 1994, 45, 1-158. (c) Giese, S.; West, F. G. Tetrahedron 2000, 56, 10221. (d) Wang, Y.; Schill, B. D.; Arif, A. M.; West, F. G. Org. Lett. 2003, 5, 2747. (e) Aggarwal, V. K.; Beffield, A. J. Org. Lett. 2003, 5, 5075. (f) Bee, C.; Leclerc, E.; Tius, M. A. Org. Lett. 2003, 5, 4927. (g) He, W.; Sun, X. F.; Frontier, A. J. J. Am. Chem. Soc. 2003, 125, 14278. (h) Janka, M.; He, W.; Frontier, A. J.; of the Nazarov cyclization was recognized at an early stage in organic synthesis. Solutions to control the termination of the reaction were devised several decades ago, ${ }^{3 b}$ but the necessity of using a stoichiometric amount of strong Lewis or Brønsted acids has limited the use of this reaction. However, in the last five years the first examples of catalytic Nazarov reactions using milder Lewis $^{3 \mathrm{c}-3 \mathrm{n}}$ or Brønsted ${ }^{30,3 \mathrm{p}}$ acids were reported, together with the first examples of asymmetric induction. ${ }^{3 e, 3 k, 31,3 n, 30}$

Eisenberg, R. J. Am. Chem. Soc. 2004, 126, 6864. (i) Malona, J. A. Colbourne, J. M.; Frontier, A. J. Org. Lett. 2006, 8, 5661. (j) He, W.; Herrick, I. R.; Atesin, T. A; Caruana, P. A.; Kellenberger, C. A.; Frontier, A. J. J. Am. Chem. Soc. 2008, 130, 1003. (k) Liang, G. X.; Gradl, S. N.; Trauner, D. Org. Lett. 2003, 5, 4931. (l) Liang, G. X., Trauner D. J. Am. Chem. Soc. 2004, 126, 9544. (m) Walz, I.; Bertogg, A.; Togni, A. Eur. J. Org. Chem. 2007, 2650. (n) Walz, I.; Togni, A. Chem. Commun. 2008, 4315. (o) Rueping, M.; Ieawsuwan, W.; Antonchick, A. P.; Nachtsheim, B. J. Angew. Chem., Int. Ed. 2007, 46, 2097. (p) Amere, M.; Blanchet, J.; Lasne, M. C.; Rouden, J. Tetrahedron Lett. 2008, 49, 2541. For reviews, see: (q) Tius, M. A. Eur. J. Org. Chem. 2005, 2193. (r) Frontier, A. J.; Collison, C. Tetrahedron 2005, 61, 7577. (s) Pellissier, H. Tetrahedron 2005, 61, 6479. 
A) Nazarov<smiles>[R]C=C([X])C(=O)C([R])=C[R]</smiles>

B) Homo-Nazarov<smiles>[R]C=C([X])C(=O)C1([R])CC1[R]</smiles>

When considering these recent successes in the Nazarov reaction, we wondered if similar concepts could be successful in other cyclization reactions to access larger ring systems. A viable approach to access homologous rings via electrocyclic reactions is the substitution of a double bond by a cyclopropyl group, as exemplified by the divinylcyclopropyl rearrangement. ${ }^{4}$ Intra- and intermolecular ring-opening of cyclopropyl ketones and diesters have been examined extensively. ${ }^{5}$ The reaction of vinyl-cyclopropyl ketones has been less studied (B, Scheme 1). ${ }^{6}$ Tsuge has reported the cyclization of vinyl-cyclopropyl ketones using an excess of polyphosphoric acid at $80^{\circ} \mathrm{C}$, but this reaction was not general and several other products were obtained beside the desired cyclohexenones. ${ }^{6 a}$ More work has been done on the related aryl-cyclopropyl ketones, first by Murphy for the synthesis of tetralones using an excess of $\mathrm{SnCl}_{4}$ as reagent. ${ }^{6 b-6 d}$ During completion of our work, Yadav also demonstrated that diverse polycyclic heterocycles could be accessed using 3 equivalents $\mathrm{SnCl}_{4}$ at $80^{\circ} \mathrm{C}$, but no vinyl-cyclopropyl ketones were reported. ${ }^{6 e} \mathrm{Up}$ to now, the harsh conditions needed have limited the use of the homoNazarov reaction in organic synthesis. Herein, we report the first example of a catalytic formal homo-Nazarov process for non aromatic substrates which lead to the formation of valuable polycyclic cyclohexenones at room temperature as well as preliminary experiments to probe the reaction mechanism.

Inspired by recent progress in the catalytic Nazarov reaction, ${ }^{3 j}$ we decided to examine dihydropyran-derived substrate 2a (Scheme 2). Substrate 2a was synthesized from Weinreb amide $\mathbf{1}$ via Corey-Chaykovsky

(4) Piers, E. In Comprehensive Organic Synthesis; Trost, B. M., Ed.; Pergamon Press: New York, 1991; Vol. 5, p 971-998.

(5) For a few selected examples, see: (a) Stork, G.; Marx, M. J. Am. Chem. Soc. 1969, 91, 2371. (b) Grieco, P. A.; Finkelhor, R. S. Tetrahedron Lett. 1974, 527. (c) Pohlhaus, P. D.; Sanders, S. D.; Parsons, A. T.; Li, W.; Johnson, J. S. J. Am. Chem. Soc. 2008, 130, 8642.

(6) (a) Tsuge, O.; Kanemasa, S.; Otsuka, T.; Suzuki, T. Bull. Chem. Soc. Jpn. 1988, 61, 2897. (b) Murphy, W. S.; Wattanasin, S. Tetrahedron Lett. 1980, 21, 1887. (c) Murphy, W. S.; Wattanasin, S. J. Chem. Soc. Perkin Trans. 1 1981, 2920. (d) Murphy, W. S.; Wattanasin, S. J. Chem. Soc. Perkin Trans. 1 1982, 1029. (e) Yadav, V. K.; Kumar, N. V. Chem. Commun. 2008, 3774.
Scheme 2. Synthesis and Cyclization of Model Substrate 2a.

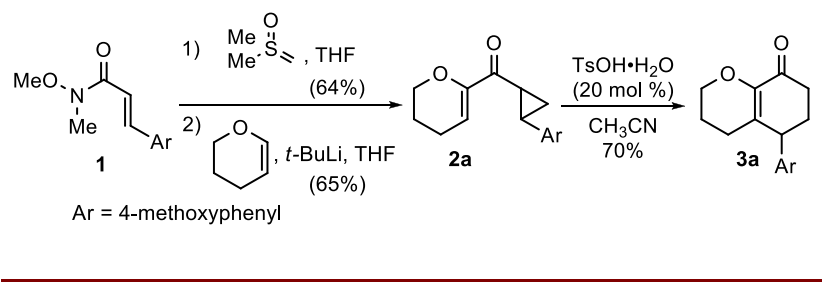

With our model substrate in hand, we began our studies by examining the most frequently used procedure for homo-Nazarov cyclization: stoichiometric $\mathrm{SnCl}_{4}{ }^{6 \mathrm{~b}-6 \mathrm{e}}$ Using these conditions, complete polymerization of the sensitive substrate was observed. ${ }^{8}$

As most Lewis acid led to extensive polymerization, we then turned towards Brønsted acid catalysts. The $\mathrm{pK}_{\mathrm{a}}$ value of the catalyst had a strong influence on the outcome of the reaction: Sulfuric and toluenesulfonic acids were optimal. Stronger acids led to decomposition of the starting material and no full conversion could be achieved with weaker acids. Examination of solvent effects showed that the reaction was faster in noncoordinating solvents, like dichloromethane, but polymerization was also difficult to suppress. Acetonitrile finally offered the best compromise, with sufficient reactivity but less pronounced polymerization. The cyclization of $\mathbf{2 a}$ in acetonitrile with $20 \mathrm{~mol} \%$ toluenesulfonic acid at room temperature led to the formation of the desired cyclohexenone 3a in 70\% isolated yield (Scheme 2).

The scope of the reaction was examined next (Table 1). Variation of the aromatic substituent on the cyclopropane confirmed the importance of its electron-donating ability: whereas no reaction was observed with a simple phenyl group (entry 2), a quantitative yield was observed with a 3,4- or 2,4- dimethoxyphenyl group (entries 3 and 4). This result is noteworthy, as electron-rich aromatic substituents are well represented in bioactive natural products ${ }^{9}$ and are easily oxidized to the corresponding carboxylic acids. ${ }^{10} \mathrm{~A}$ furan group was also tolerated at this position, although the yield was moderate due to partial polymerization (entry 5 ).

Finally, the influence of a methyl group $\alpha$ to the ketone was examined. Interestingly, a strong accelerating effect

(7) Rodriques, K. E. Tetrahedron Lett. 1991, 32, 1275.

(8) Oligomerization, then polymerization was apparent in ${ }^{1} \mathrm{HNMR}$ via formation of broad signals in several regions of the spectra, see Supporting Information (Figure S5).

(9) For example in Podophyllotoxin natural products and their derivatives: Bohlin, L.; Rosen, B. Drug Discov. Today 1996, 1, 343.

(10) (a) Carlsen, P. H. J.; Katsuki, T.; Martin, V. S.; Sharpless, K. B. J. Org. Chem. 1981, 46, 3936. (b) Voight, E. A.; Rein, C., Burke, S. D J. Org. Chem. 2002, 67, 8489. 
was observed and cyclohexenone $3 f$ was obtained in quantitative yield after only 15 min (entry 6).

Table 1. Scope of the formal homo-Nazarov Cyclization

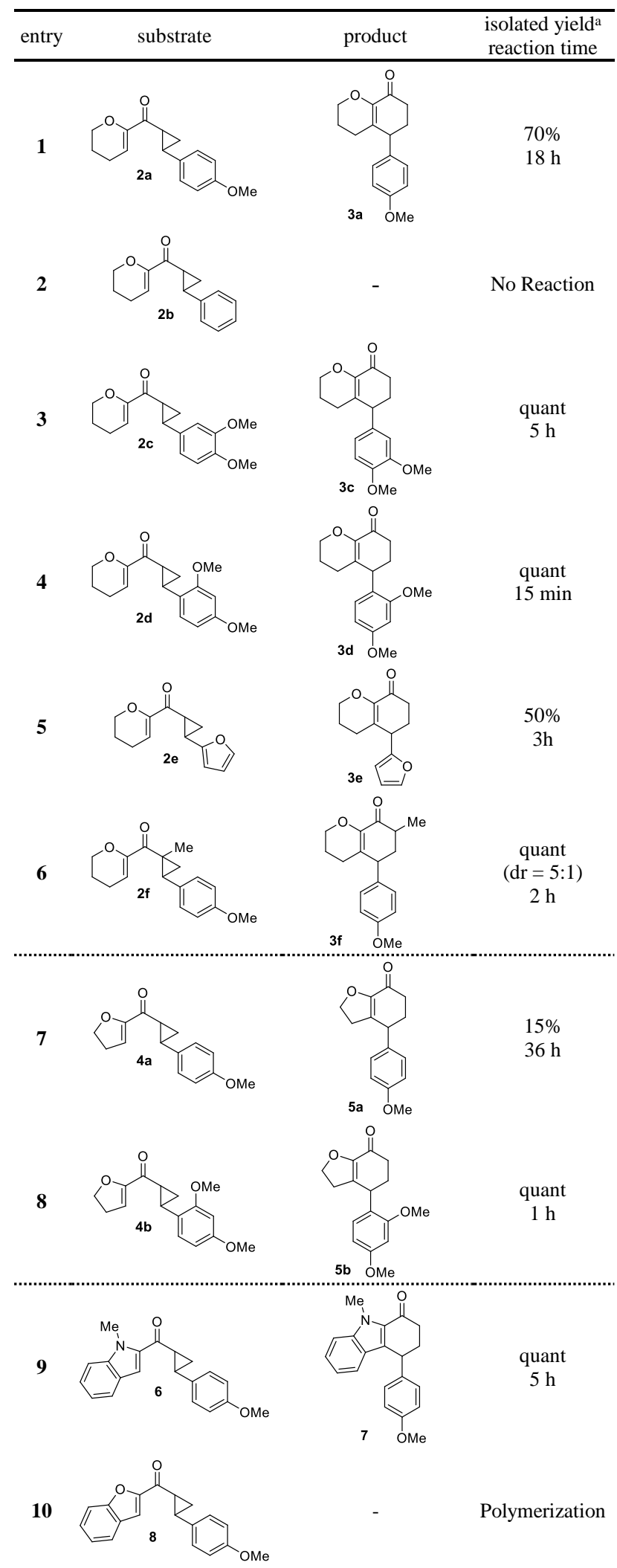

a Reaction conditions: $0.4 \mathrm{mmol}$ substrate in $8 \mathrm{~mL} \mathrm{CH}_{3} \mathrm{CN}$ with 20 $\mathrm{mol} \% \mathrm{TsOH}$ at $23^{\circ} \mathrm{C}$.
A plausible explanation would be a faster ring-opening of the cyclopropane ring due to sterical strain release and the higher stability of the formed enol intermediate. ${ }^{11}$ Importantly, this accelerating effect on the formal homoNazarov cyclization has never been reported before.

We then examined variation of the electron-rich side of the ketone. A dihydrofuran group proved to be more prone to polymerization and the desired product was isolated only in low yield with a 4-methoxyphenyl group on the cyclopropane (entry 7). The stronger stabilizing effect of the 2,4-dimethoxyphenyl substituent allowed the isolation of the desired 5-6 ring system in quantitative yield (entry 8). Replacing the dihydropyran group with an electron-rich $N$-methylindole heterocycle lead to an efficient cyclization in quantitative yield (entry 9), but only polymerization was observed with a benzofuran ring (entry 10). Interestingly, similar results were obtained in the related Nazarov cyclization. ${ }^{3 \mathrm{i}}$

In order to further increase the versatility of the formal homo-Nazarov process, it would be important to diminish the strong electronic constraints which limit the number of structures that can be synthesized. The use of substrates lacking an electron-donating hetero atom on the doublebond is highly desirable. Based on the seminal work of Denmark on silyl group-directed Nazarov reactions, ${ }^{3 b, 12}$ we decided to use an allyl silane group to enhance the nucleophilicity of the double bond and favorize cyclization (Scheme 3).

Scheme 3. Cyclization for Carbocycles Synthesis

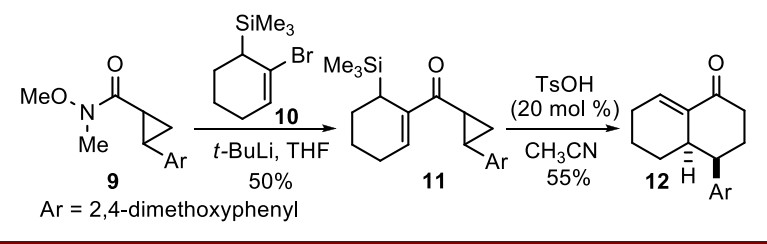

Gratifyingly, submitting vinyl-cyclopropyl ketone $\mathbf{1 1}$ to the optimized reaction conditions led to the formation of bicyclic ketone 12 in $30 \mathrm{~min}$ and $55 \%$ yield. The regioselectivity of the double bond formation was completely controlled by the elimination of the silyl group. Interestingly, only one diastereoisomer of $\mathbf{1 2}$ was isolated. The structure of $\mathbf{1 2}$ was tentatively assigned by NMR experiments (COSY, HSQC, NOESY). ${ }^{13}$ This preliminary result held promises for the application of the method in the synthesis of carbocyclic compounds.

The strong influence of electron-donating groups and acid strength on the reaction rate led us to propose a

(11) As an alternative explanation, a higher fraction of the more stable enol tautomer could be envisaged to favor cyclization.

(12) Denmark, S. E.; Klix, R. C. Tetrahedron 1988, 44, 4043.

(13) The obtained 2D NMR data strongly support the proposed structure assignment for 12. Further confirmation of the structure will be attempted by X-rays analysis of the corresponding thiosemicarbazone, a procedure developed by Denmark. ${ }^{12}$ 
tentative stepwise mechanism for the reaction with cyclopropane opening as rate-limiting (Scheme 4).

Scheme 4. Postulated Mechanism

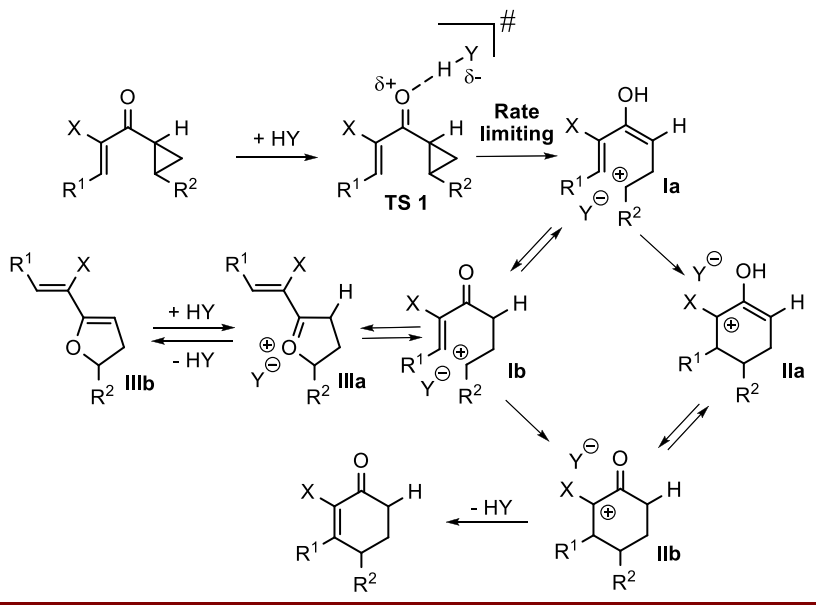

In order to further support this mechanism, the following experiments were performed (Scheme 5): (1) The reaction kinetic was followed via ${ }^{1} \mathrm{H}$ NMR spectroscopy, and the reaction was found to be first order in tosic acid for substrate 2a. (2) The use of stoichiometric deuterated tosic acid resulted in a mixture of non-deuterated, mono and bis-deuterated products at the $\alpha$ position to the ketone. A control experiment showed that no deuterium exchange was observed for the isolated cyclization product in the presence of deuterated tosic acid. A possible explanation for this surprising results would be the intramolecular attack of the oxygen atom of intermediates Ia or $\mathbf{I b}$ to form an oxonium intermediate IIIa. From IIIa, proton-deuterium exchange should be easy via dihydrofuran IIIb. Dihydrofuran products have indeed been isolated in the related stoichiometric reaction of aryl vinyl ketones. ${ }^{6 c}$ Alternatively, proton exchange could be more rapid on cyclized intermediate IIb and IIa. Proton lost form IIb, or proton lost followed by tautomerization from IIa would then lead to the cyclohexenone product. Additionally, a weak kinetic isotope effect (1.15) was observed using $40 \mathrm{~mol} \%$ deuterated tosic acid, but this result is difficult to interpret due to fast proton exchange between substrate and catalyst. (3) Attempts were made to trap the proposed intermediates (enol and carbocation) of the catalytic cycle. ${ }^{14}$ With substrate 2a, all nucleophilic (water, allyl silane, butyl vinyl ether) and electrophilic (benzaldehyde, ethyl glyoxalate, acetic anhydride, ethyl acrylate) trapping agents tested so far were not successful. With cyclohexene derivative 15, however, alcohol $\mathbf{1 6}$ was obtained in $31 \%$ yield when the reaction was conducted in the presence of water.

(14) To gain stronger evidence for the cationic mechanism, we will study the influence of the cyclopropane stereochemistry on the reaction outcome, for example using 11 with a cis-substituted cyclopropane.

All the data collected so far are in agreement with a rate-determining cyclopropane opening, followed by a fast cyclization. In the case were the cyclization is too slow (as with 15), polymerization can occur instead of the desired process. We speculate that key for catalysis is the fast tautomerization of the enol intermediates, which contrasts with the strong binding of stoichiometric reagents like $\mathrm{SnCl}_{4}$, which prevent catalytic turnover.

Scheme 5. Mechanism Investigation: (1) Van't Hoff Plot and Reaction Order (2) Deuterium and (3) Trapping Experiments

(1)

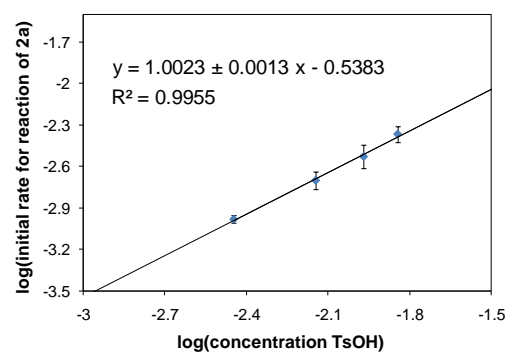

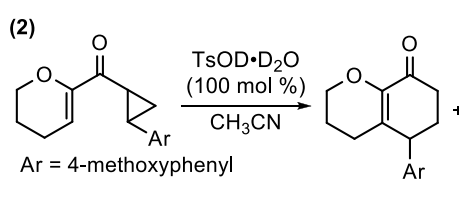

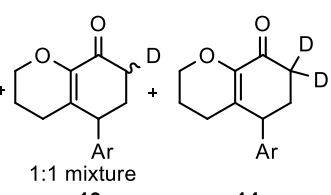

14 3a:13:14 = 0.9:1:0.7

(3)

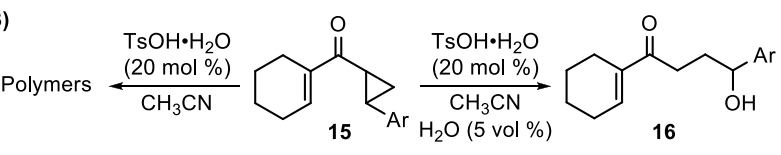
$\mathrm{Ar}=4$-methoxyphenyl $32 \%$

In summary, we have reported the first catalytic formal homo-Nazarov process. We have demonstrated that principles successful in the corresponding Nazarov reaction could also be applied to vinyl-cyclopropyl ketones, which allow the first high-yielding cyclization reaction for this class of substrates under mild conditions. First investigations of the reaction mechanism seem to indicate a stepwise mechanism with a rate-limiting cyclopropane ring opening: consequently, the reaction is mechanistically different from the classical Nazarov cyclization. Our future work will focus on the development of asymmetric variations as well as on applications in the synthesis of natural products and their analogs.

Acknowledgment The EPFL is acknowledged for financial support. We thank Prof. K. Gademann, Dr. T. Woods and Dr. H. Jessen from the Chemical Synthesis Laboratory at the EPFL for proofreading this manuscript.

Supporting Information Available Experimental procedures, spectroscopic information for new 
compounds and kinetic data. This material is available free of charge via the Internet at http://pubs.acs.org 


\section{Supporting information \\ Catalytic Formal Homo-Nazarov Cyclization}

Filippo De Simone, Julien Andrès, Riccardo Torosantucci and Jérôme Waser*

57 pages

1. General Methods S2

2. General procedures $\quad \mathrm{S} 2$

3. Substrate synthesis S3

4. Cyclization $\quad \mathrm{S} 15$

5. Kinetic Data S20

6. Spectra S24 


\section{General Methods}

All reactions were carried out in oven dried glassware under an atmosphere of nitrogen, unless stated otherwise. For quantitative flash chromatography technical grade solvents were used. For flash chromatography for analysis, HPLC grade solvents from Sigma-Aldrich were used. THF, $\mathrm{Et}_{2} \mathrm{O}, \mathrm{CH}_{3} \mathrm{CN}_{\text {, }}$ toluene, hexane and $\mathrm{CH}_{2} \mathrm{Cl}_{2}$ were dried by passage over activated alumina under nitrogen atmosphere $\left(\mathrm{H}_{2} \mathrm{O}\right.$ content $<10 \mathrm{ppm}$, Karl-Fischer titration). $\mathrm{NEt}_{3}$ and pyridine were distilled under nitrogen from $\mathrm{KOH}$. All chemicals were purchased from Acros, Aldrich, Fluka, VWR, Aplichem or Merck and used as such unless stated otherwise. Chromatographic purification was performed as flash chromatography using MachereyNagel silica 40-63, $60 \AA$, using the solvents indicated as eluent with 0.1-0.5 bar pressure.TLC was performed on Merck silica gel $60 \mathrm{~F}_{254}$ TLC glass plates or aluminium plates and visualized with UV light, permanganate stain, CAN stain or Anisaldehyde stain.Melting points were measured on a Büchi B-540 melting point apparatus using open glass capillaries, the data is uncorrected. ${ }^{1} \mathrm{H}-\mathrm{NMR}$ spectra were recorded on a Brucker DPX-400 $400 \mathrm{MHz}$ spectrometer in chloroform-d, DMSO- $\mathrm{d}_{6}$ or $\mathrm{CD}_{3} \mathrm{OD}$, all signals are reported in ppm with the internal chloroform signal at $7.26 \mathrm{ppm}$, the internal DMSO signal at $2.50 \mathrm{ppm}$ or the internal methanol signal at $3.30 \mathrm{ppm}$ as standard. The data is being reported as $(\mathrm{s}=$ singlet, $\mathrm{d}=$ doublet, $\mathrm{t}=$ triplet, $\mathrm{q}=$ quadruplet, $\mathrm{qi}=$ quintet, $\mathrm{m}=$ multiplet or unresolved, $\mathrm{br}=$ broad signal, $\mathrm{app}=$ apparent, coupling constant(s) in $\mathrm{Hz}$, integration; interpretation) ${ }^{13} \mathrm{C}-\mathrm{NMR}$ spectra were recorded with ${ }^{1} \mathrm{H}$ decoupling on a Brucker DPX-400 $100 \mathrm{MHz}$ spectrometer in chloroform-d, DMSO- $\mathrm{d}_{6}$ or $\mathrm{CD}_{3} \mathrm{OD}$, all signals are reported in ppm with the internal chloroform signal at $77.0 \mathrm{ppm}$, the internal DMSO signal at $39.5 \mathrm{ppm}$ or the internal methanol signal at $49.0 \mathrm{ppm}$ as standard. Infrared spectra were recorded on a JASCO FT-IR B4100 spectrophotometer with an ATR PRO410-S and a ZnSe prisma and are reported as $\mathrm{cm}^{-1}(\mathrm{w}=$ weak, $\mathrm{m}=$ medium, $\mathrm{s}=$ strong, $\mathrm{br}=$ broad). Gas chromatographic and low resolution mass spectrometric measurements were performed on a Perkin-Elmer Clarus 600 gas chromatographer and mass spectrometer using a Perkin-Elmer Elite fused silica column (length: $30 \mathrm{~m}$, diameter: $0.32 \mathrm{~mm}$ ) and Helium as carrier gas. High resolution mass spectrometric measurements were performed by the mass spectrometry service of ISIC at the EPFL on a MICROMASS (ESI) Q-TOF Ultima API. HPLC measurement were done on a JASCO HPLC system with an AS2055 Autosampler, a PU 2089 Pump, a UV 2075 detector and a SEDEX 85 (SEDERE) detector using a CHIRALPAK IC column from DAICEL Chemical Industries Ltd. HPLC grade solvents from Sigma-Aldrich were used.

\section{General Procedures}

\section{General procedure 1: formation of Weinreb’s amides}

Following the reported procedure ${ }^{1} N$-methylmorpholine (1.1 equiv) was added to a solution of acid (1.0 equiv) in DMF $(1 \mathrm{M})$ at $0{ }^{\circ} \mathrm{C}$. After $25 \mathrm{~min}$, iso-butylchloroformate (1.1 equiv) was added dropwise at $0{ }^{\circ} \mathrm{C}$. After 10 min, $\mathrm{N}, \mathrm{O}$-dimethylhydroxylamine hydrochloride (1.1 equiv) was added, followed by $N$ methylmorpholine (1.3 equiv) and the reaction mixture was warmed to $23^{\circ} \mathrm{C}$. After $6 \mathrm{~h}$, the reaction was quenched with $0.5 \mathrm{M} \mathrm{HCl}(2 \mathrm{~mL} / \mathrm{mmol}$ of acid $)$ and extracted with $\mathrm{CH}_{2} \mathrm{Cl}_{2}(3 \times 2 \mathrm{~mL} / \mathrm{mmol}$ of acid $)$. The combined organic layers were washed with $0.5 \mathrm{~m} \mathrm{NaOH}(2 \times 2 \mathrm{~mL} / \mathrm{mmol}$ of acid), brine $(2 \mathrm{~mL} / \mathrm{mmol}$ of acid), dried over $\mathrm{MgSO}_{4}$ and the solvent was removed under reduced pressure. After $30 \mathrm{~min}$ in high vacuum, the residues were dissolved in $\mathrm{Et}_{2} \mathrm{O}(6 \mathrm{~mL} / \mathrm{mmol}$ of acid) and washed with brine $(2 \times 3 \mathrm{~mL} / \mathrm{mmol}$ of acid),

\footnotetext{
${ }^{1}$ Nagarajan, S. R.; Lu, H. F.; Gasiecki, A. F.; Khanna, I. K.; Parikh, M. D.; Desai, B. N.; Rogers, T. E.; Clare, M.; Chen, B. B.; Russell, M. A.; Keene, J. L.; Duffin, T.; Engleman, V. W.; Finn, M. B.; Freeman, S. K.; Klover, J. A.; Nickols, G. A.; Nickols, M. A.; Shannon, K. E.; Steininger, C. A.; Westlin, W. F.; Westlin, M. M.; Williams, M. L. Bioorg. Med. Chem. 2007, 15, 3390-3412.
} 
dried over $\mathrm{MgSO}_{4}$ and the solvent was removed under reduced pressure to give the Weinreb amide which was used directly without purification.

\section{General procedure 2: formation of Corey-Chaykovsky ylide}

$n \operatorname{BuLi}(2.5 \mathrm{M}, 1.0$ equiv) was added dropwise to a solution of trimethylsufoxonium iodide (1.1 equiv) in anhydrous THF $(0.75 \mathrm{~m})$ at $0^{\circ} \mathrm{C}$. The solution was allowed to warm to RT and stirring was continued under nitrogen for 1 hour. A solution $0.54 \mathrm{~m}$ of ylide was obtained.

\section{General procedure 3: Cyclopropanation 1 (Corey-Chaykovsky)}

A solution of ylide (1.1 equiv) in anhydrous THF $(0.54 \mathrm{M})$ was added dropwise to a solution of the alkene derivative (1.0 equiv) in anhydrous THF $(0.10 \mathrm{M})$ at RT under nitrogen. The mixture was stirred at the indicated temperature during the indicated time. The reaction was quenched with $\mathrm{NaHCO}_{3}(10$ $\mathrm{mL} / \mathrm{mmol})$ and extracted with $\mathrm{Et}_{2} \mathrm{O}(3 \times 10 \mathrm{~mL} / \mathrm{mmol})$. The combined organic layers were washed with brine (2x10 $\mathrm{mL} / \mathrm{mmol})$, dried over $\mathrm{MgSO}_{4}$ and the solvent was removed under reduced pressure.

\section{General procedure 4: formation of ketone from Weinreb’s amide}

Following a slight modification of a reported procedure, ${ }^{2} t \mathrm{BuLi}$ (2.0 equiv) was added dropwise in a solution of alkene derivative $\left(2.2\right.$ equiv) in THF $(0.10 \mathrm{M})$ at $-78^{\circ} \mathrm{C}$. The flask was transferred in a bath of ice. After the indicated time the reaction was cooled to $-78^{\circ} \mathrm{C}$ and a solution of amide (1.0 equiv) in THF $(0.20$ M) was added slowly dropwise. The reaction was stirred at $-78^{\circ} \mathrm{C}$ during the indicated time and controlled via TLC. The solution was finally warmed at $0^{\circ} \mathrm{C}$ and quenched with saturate solution of $\mathrm{NH}_{4} \mathrm{Cl}(5$ $\mathrm{mL} / \mathrm{mmol})$. The product was extracted with $\mathrm{Et}_{2} \mathrm{O}(10 \mathrm{~mL} / \mathrm{mmol}$ of amide $)$ and washed with brine $(2 \times 6$ $\mathrm{mL} / \mathrm{mmol}$ of amide), dried over $\mathrm{MgSO}_{4}$ and concentrated under reduced pressure.

\section{General procedure 5: cyclization}

Toluenesulphonic acid ( $15 \mathrm{mg}, 80 \mu \mathrm{mol}, 0.20$ equiv) was added to a solution of a vinyl cyclopropyl ketone derivative $\left(0.40 \mathrm{mmol}, 1.0\right.$ equiv) in anhydrous $\mathrm{CH}_{3} \mathrm{CN}(10 \mathrm{~mL})$ at room temperature. The reaction was stirred during the indicated time. The solution was quenched with $\mathrm{NaHCO}_{3}(10 \mathrm{~mL})$ and extracted with $\mathrm{Et}_{2} \mathrm{O}(3 \times 10 \mathrm{~mL})$. The combined organic layers were washed with brine $(2 \times 10 \mathrm{~mL})$, dried over $\mathrm{MgSO}_{4}$ and the solvent was removed under reduced pressure.

\section{Substrates synthesis}

\section{2-Acetyl-5,6-dihydro-4H-pyran (17)}
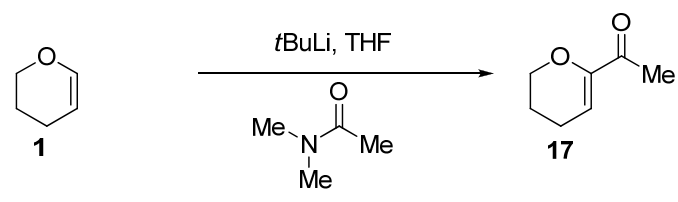

Following a reported procedure, ${ }^{2}$ a $1.5 \mathrm{~m}$ solution of $t \mathrm{BuLi}$ in pentane $(16 \mathrm{~mL}, 24 \mathrm{mmol} 1.0$ equiv) was added dropwise to a solution of 5,6-dihydro- $4 H$-pyran $(2.2 \mathrm{~mL}, 24 \mathrm{mmol}, 1.0$ equiv) in THF (15 mL) at $78^{\circ} \mathrm{C}$. The reaction mixture was warmed to $0^{\circ} \mathrm{C}$ and stirred during $30 \mathrm{~min}$ then cooled to $-78^{\circ} \mathrm{C}$. A solution

\footnotetext{
${ }^{2}$ Boeckman, R. K.; Bruza, K. J. Tetrahedron 1981, 37, 3997-4006.
} 
of dimethylacetamide ( $2.9 \mathrm{~mL}, 32 \mathrm{mmol}, 1.3$ equiv) in THF $(2.0 \mathrm{~mL})$ was added dropwise during $5 \mathrm{~min}$ and the reaction was allowed to warm slowly to RT. After $2 \mathrm{~h}$ the reaction was quenched with an aqueus saturated solution of $\mathrm{NH}_{4} \mathrm{Cl}(10 \mathrm{~mL})$ and extracted with $\mathrm{Et}_{2} \mathrm{O}(20 \times 2 \mathrm{~mL})$. The organic layers were combined and washed with sat $\mathrm{NaCl}$, dried on $\mathrm{MgSO}_{4}$ and evaporated under reduced pression. The crude product was purified by flash column chromatography (PET/AcOEt 3:1) to yield ketone 17 (2.4 g, $19 \mathrm{mmol}, 80 \%)$ as yellow oil. $R_{f} 0.60$ (DCM/AcOEt 16:1, Anisaldehyde). ${ }^{1} \mathrm{H} \mathrm{NMR}\left(\mathrm{CDCl}_{3}, 400 \mathrm{MHz}\right) \delta 5.93(\mathrm{t}, J=4.2 \mathrm{~Hz}$, 1H; CH-alkene), 4.04 (t, $J=4.9 \mathrm{~Hz}, 2 \mathrm{H} ; \mathrm{CH}_{2} \mathrm{O}$ ), 2.21 (s, 3H; $\left.\mathrm{CH}_{3}\right), 2.17$ (app dd, $J=6.3,10.7 \mathrm{~Hz}, 2 \mathrm{H}$; $\mathrm{CH}_{2}$ pyran), 1.80 (app dt, $J=6.1,12.0 \mathrm{~Hz}, 2 \mathrm{H} ; \mathrm{CH}_{2}$ pyran). ${ }^{1} \mathrm{H}$ NMR spectra corresponded to the literature values. $^{2}$

(E)-2-[2-(4-Methoxyphenyl)-1-ethylenecarbonyl]-5,6-dihydro-4H-pyran (19)

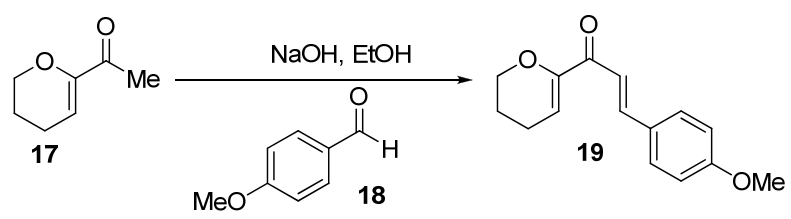

Following a slight modification of the reported procedure, ${ }^{3} \mathrm{NaOH}(0.4 \mathrm{~mL}, 2.5 \mathrm{~m})$ was added to a solution of $17(0.50 \mathrm{~g}, 4.0 \mathrm{mmol}, 1.0$ equiv) in EtOH $(8 \mathrm{~mL})$ at RT. The reaction was stirred for $5 \mathrm{~min}$ then p-anisaldehyde $18(0.50 \mathrm{~mL}, 4.0 \mathrm{mmol}, 1.0$ equiv) was added dropwise. The solution was quenched after $1 \mathrm{~h}$ and $30 \mathrm{~min}$ with water $(10 \mathrm{~mL})$ and extracted with $\mathrm{Et}_{2} \mathrm{O}(20 \times 2 \mathrm{~mL})$. The organic layer was washed with brine, dried over $\mathrm{MgSO}_{4}$, concentrated and the crude product was purified by flash column chromatography (PET/AcOEt 4:1) to yield 19 (40 mg, $0.14 \mathrm{mmol}, 50 \%$ ) as yellow oil. $R_{f} 0.70$ (PET/AcOEt 4:1, Anisaldehyde). ${ }^{1} \mathrm{H}$ NMR $\left(\mathrm{CDCl}_{3}, 400 \mathrm{MHz}\right) \delta 7.72(\mathrm{~d}, J=15.8 \mathrm{~Hz}, 1 \mathrm{H} ; \mathrm{CH}-\mathrm{Ar}), 7.56(\mathrm{~d}, J=8.7 \mathrm{~Hz}, 2 \mathrm{H}$; Ar-H), $7.22(\mathrm{~d}, J=15.8 \mathrm{~Hz}, 1 \mathrm{H}$; CHCO), 6.90 (d, $J=8.7 \mathrm{~Hz}, 2 \mathrm{H}$; Ar-H), 6.09 (t, $J=4.2 \mathrm{~Hz}, 1 \mathrm{H}$; alkene$\mathrm{H}), 4.15\left(\mathrm{t}, J=5.2 \mathrm{~Hz}, 2 \mathrm{H} ; \mathrm{CH}_{2} \mathrm{O}\right), 3.84\left(\mathrm{~s}, 3 \mathrm{H} ; \mathrm{OCH}_{3}\right), 2.26$ (app dd, $J=6.3,10.7 \mathrm{~Hz}, 2 \mathrm{H} ; \mathrm{CH}_{2}$ pyran), $1.96-1.85$ (m, 2H; $\mathrm{CH}_{2}$ pyran). ${ }^{13} \mathrm{C} \mathrm{NMR}\left(\mathrm{CDCl}_{3}, 100 \mathrm{MHz}\right) \delta 184.7,161.2,151.6,143.3,130.0,127.3$, 117.8, 114.0, 109.9, 66.0, 55.0, 21.3, 20.6. IR v $2953(\mathrm{w}), 2934$ (w), $2837(\mathrm{w}), 1660(\mathrm{w}), 1627(\mathrm{~m}), 1589(\mathrm{~s})$, 1570 (s), 1510 (s), 1423 (m), 1327 (m), 1295 (m), 1281 (m), 1253 (s), 1235 (s), 1200 (m), 1171 (s), 1090 (m), 1059 (s), 1027 (s), $986(\mathrm{~m}), 918$ (s), 829 (s), 800 (m), 773 (m), 714 (m). HRMS(ESI) calcd for $\mathrm{C}_{15} \mathrm{H}_{16} \mathrm{O}_{3}^{+}(\mathrm{M}+\mathrm{H})$ 245.1172, found 245.1178.

(E)- $N$-Methoxy- $N$-methyl-3-(4-methoxyphenyl)-acrylamide (1) and $N$-methoxy- $N$-methyl-1-[2-(4methoxyphenyl)-cyclopropan-1-yl]-formamide (21)

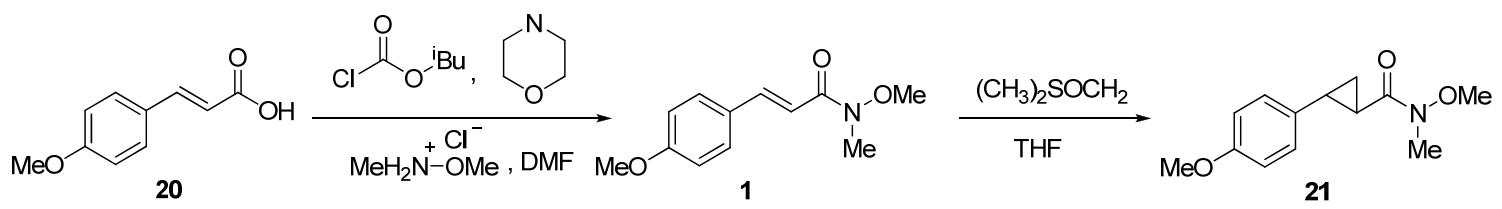

Following general procedure 1, the acid 20 (2.00 g, $11.2 \mathrm{mmol}, 1.00$ equiv) gave the Weinreb amide 1 which was used directly without purification. Using general procedure 3, a solution of ylide $(12.2 \mathrm{~mL}, 6.58$ mmol, $1.20 \mathrm{eq})$ was added to a solution of amide 1 (1.21 g, $5.47 \mathrm{mmol}, 1.00$ equiv) in THF (45 mL). The reaction was stirred at $40^{\circ} \mathrm{C}$ during $2 \mathrm{~h}$ then quenched. Purification by flash column chromatography (PET/AcOEt, 7:3) afforded 21 (824 mg, $3.50 \mathrm{mmol}, 64 \%$ ) over 2 steps as oil. $R_{f} 0.35$ (PET/AcOEt 7:3, Anisaldehyde). ${ }^{1} \mathrm{H}$ NMR $\left(\mathrm{CDCl}_{3}, 400 \mathrm{MHz}\right) \delta 7.06$ (d, $J=8.6 \mathrm{~Hz}, 2 \mathrm{H}$; Ar-H), 6.83 (d, $J=8.7 \mathrm{~Hz}, 2 \mathrm{H}$; Ar$\mathrm{H}), 3.78\left(\mathrm{~s}, 3 \mathrm{H} ; \mathrm{OCH}_{3}\right), 3.69\left(\mathrm{~s}, 3 \mathrm{H} ; \mathrm{OCH}_{3}\right), 3.23\left(\mathrm{~s}, 3 \mathrm{H} ; \mathrm{NCH}_{3}\right), 2.50-2.42(\mathrm{~m}, 1 \mathrm{H}$; cyclopropane $\mathrm{CH})$,

${ }^{3}$ Matsui, M.; Oji, A.; Hiramatsu, K.; Shibata, K.; Muramatsu, H. J. Chem. Soc.-Perkin Trans. 2 1992, 201-206. 
$2.33(\mathrm{~m}, 1 \mathrm{H}$; cyclopropane $\mathrm{CH}), 1.65-1.55\left(\mathrm{~m}, 1 \mathrm{H}\right.$; cyclopropane $\left.\mathrm{CH}_{2}\right), 1.30-1.22(\mathrm{~m}, 1 \mathrm{H}$; cyclopropane $\left.\mathrm{CH}_{2}\right) .{ }^{1} \mathrm{H}$ NMR spectra corresponded to the literature values. ${ }^{1}$

\section{(E)-2-[2-(4-Methoxyphenyl)-1-cyclopropanecarbonyl]-5,6-dihydro-4H-pyran (2a)}

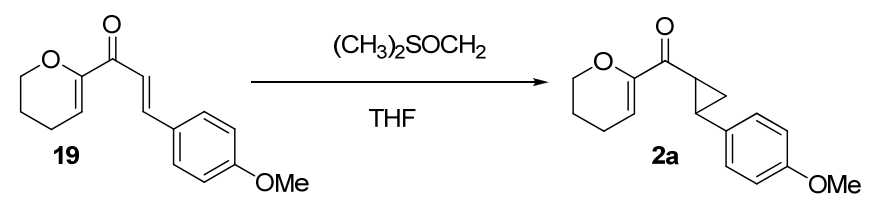

Following general procedure 3, a solution of sulphoxonium ylide $(2.90 \mathrm{~mL}, 1.57 \mathrm{mmol}, 1.20$ equiv) was added to a solution of alkene 19 ( $0.35 \mathrm{~g}, 1.4 \mathrm{mmol}, 1.0$ equiv) in THF (15 mL). The reaction was stirred at RT during $3 \mathrm{~h}$ and $30 \mathrm{~min}$ then quenched. Purification by flash column chromatography (PET/AcOEt 4:1) gave 2a $(0.2 \mathrm{~g}, 0.8 \mathrm{mmol}, 55 \%)$ as yellow oil.

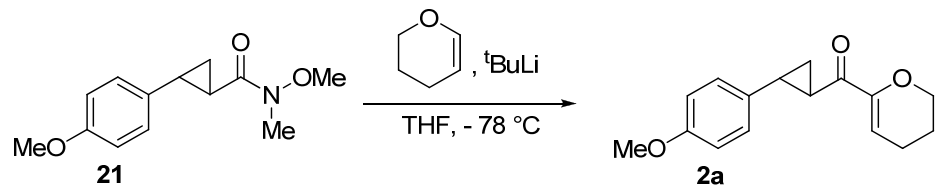

General procedure 4 was followed using dihydropyran $(0.14 \mathrm{~mL}, 1.5 \mathrm{mmol}, 2.2$ equiv) and amide 21 ( $0.16 \mathrm{~g}, 0.68 \mathrm{mmol}, 1.0$ equiv). The deprotonation time was $30 \mathrm{~min}$ at $0^{\circ} \mathrm{C}$ and the reaction was quenched after $2 \mathrm{~h}$ and $15 \mathrm{~min}$ to give 2a $(105 \mathrm{mg}, 410 \mu \mathrm{mol}, 60 \%)$ after purification via flash chromatography (PET/AcOEt, 7:3) as yellow oil. $R_{f} 0.70$ (PET/AcOEt 7:3, Anisaldehyde). ${ }^{1} \mathrm{H}$ NMR $\left(\mathrm{CDCl}_{3}, 400 \mathrm{MHz}\right) \delta$ $7.06(\mathrm{~d}, J=8.6 \mathrm{~Hz}, 2 \mathrm{H}$; Ar H), 6.83 (d, $J=8.7 \mathrm{~Hz}, 2 \mathrm{H}$; Ar-H), 6.01 (t, $J=4.2 \mathrm{~Hz}, 1 \mathrm{H}$; alkene-H), $4.14-$ $4.06\left(\mathrm{~m}, 2 \mathrm{H} ; \mathrm{CH}_{2} \mathrm{O}\right), 3.79$ (s, 3H; $\left.\mathrm{OCH}_{3}\right), 2.71-2.59$ (m, 1H; $\mathrm{CH}$ cyclopropane), 2.54 (ddd, $J=4.1,6.6$, $10.5 \mathrm{~Hz}, 1 \mathrm{H}$; $\mathrm{CH}$ cyclopropane), 2.22 (dd, $J=6.3,10.7 \mathrm{~Hz}, 2 \mathrm{H} ; \mathrm{CH}_{2}$ dihydropyran), $1.92-1.81$ (m, 2H; $\mathrm{CH}_{2}$ dihydropyran), $1.76-1.67$ (m, 1H; $\mathrm{CH}_{2}$ cyclopropane), 1.37 (ddd, $J=4.0,6.8,8.0 \mathrm{~Hz}, 1 \mathrm{H} ; \mathrm{CH}_{2}$ cyclopropane). ${ }^{13} \mathrm{C} \mathrm{NMR}\left(\mathrm{CDCl}_{3}, 100 \mathrm{MHz}\right) \delta 194.6,158.2,151.4,132.5,127.2,113.8,109.5,66.3,55.2$, 29.3, 27.30, 21.4, 20.7, 19.2. IR v $3036(\mathrm{w}), 2950(\mathrm{w}), 2934(\mathrm{w}), 2836(\mathrm{w}), 1681(\mathrm{~m}), 1667(\mathrm{~m}), 1625(\mathrm{~s})$, 1516 (s), 1440 (m), 1393 (m), 1331 (m), 1286 (s), 1248 (s), 1237 (m), 1201 (w), 1180 (s), 1091 (m), 1061 (s), 1032 (s), 999 (m), 917 (s), 822 (s), 751 (s). HRMS(ESI) calcd for $\mathrm{C}_{16} \mathrm{H}_{18} \mathrm{O}_{3}{ }^{+}(\mathrm{M}+\mathrm{H})$ 259.1329, found 259.1335 .

\section{(E)-2-[2-(Phenyl)-1-ethylenecarbonyl]-5,6-dihydro-4H-pyran (22)}

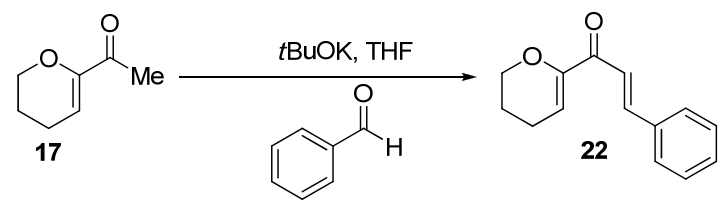

Following a slight modification of the reported procedure $4 \mathrm{BuOK}$ (44 $\mathrm{mg}, 0.40 \mathrm{mmol}, 0.10$ equiv) was added to a solution of $17\left(0.50 \mathrm{~g}, 4.0 \mathrm{mmol}, 1.0\right.$ equiv) in THF $(40 \mathrm{~mL})$ at $0^{\circ} \mathrm{C}$. The reaction was stirred at $0^{\circ} \mathrm{C}$ for $5 \mathrm{~min}$ then benzaldehyde $(0.60 \mathrm{~mL}, 6.0 \mathrm{mmol}, 1.5$ equiv) was added dropwise. The solution was quenched after $30 \mathrm{~min}$ with water $(10 \mathrm{~mL})$ and extracted with $\mathrm{Et}_{2} \mathrm{O}(2 \times 20 \mathrm{~mL})$. The organic layer was washed with brine, dried over $\mathrm{MgSO}_{4}$, concentrated and separated by flash column chromatography (PET/AcOEt 4:1) to yield 22 (39 mg, $0.14 \mathrm{mmol}, 40 \%$ ) as yellow oil. $R_{f} 0.40$ (PET/AcOEt 6:1,

${ }^{4}$ Ishikawa, T.; Aikawa, T.; Ohata, E.; Iseki, T.; Maeda, S.; Matsuo, T.; Fujino, T.; Saito, S. J. Org. Chem. 2007, 72, 435-441. 
Anisaldehyde). ${ }^{1} \mathrm{H}$ NMR $\left(\mathrm{CDCl}_{3}, 400 \mathrm{MHz}\right) \delta 7.75(\mathrm{~d}, J=15.8 \mathrm{~Hz}, 1 \mathrm{H}$; alkene-H), $7.60(\mathrm{dd}, J=2.9,6.6$ $\mathrm{Hz}, 2 \mathrm{H}$; Ph-H), 7.41 - 7.37 (m, 3H; Ph-H), 7.33 (d, $J=15.8 \mathrm{~Hz}, 1 \mathrm{H}$; alkene-H), 6.12 (t, J = 4.3 Hz, 1H; alkene-H), $4.25-4.08$ (m, 2H; $\left.\mathrm{CH}_{2} \mathrm{O}\right), 2.27$ (dd, $J=6.3,10.7 \mathrm{~Hz}, 2 \mathrm{H} ; \mathrm{CH}_{2}$ pyran), $1.98-1.82\left(\mathrm{~m}, 2 \mathrm{H} ; \mathrm{CH}_{2}\right.$ pyran). ${ }^{1} \mathrm{H}$ NMR spectra corresponded to the literature values. ${ }^{5}$

2-[2-(Phenyl)-1-cyclopropanecarbonyl]-5,6-dihydro-4H-pyran (2b)

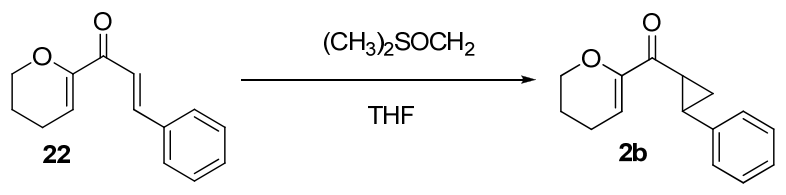

Following general procedure 3, a solution of sulphoxonium ylide (1.1 mL, $0.58 \mathrm{mmol}, 1.2$ equiv) was added to a solution of alkene $22(0.104 \mathrm{mg}, 0.485 \mathrm{mmol}, 1.00$ equiv) in THF ( $5 \mathrm{~mL})$. The reaction was stirred at RT during 15 min then quenched. Purification by flash column chromatography (PET/AcOEt 4:1) gave $2 \mathbf{b}(204 \mathrm{mg}, 0.790 \mathrm{mmol}, 50 \%)$ as colorless oil. $R_{f} 0.80$ (PET/AcOEt 7:3, Anisaldehyde). ${ }^{1} \mathrm{H}$ NMR $\left(\mathrm{CDCl}_{3}, 400 \mathrm{MHz}\right) \delta 7.28(\mathrm{dd}, J=4.7,12.4 \mathrm{~Hz}, 2 \mathrm{H} ; \mathrm{Ph}-\mathrm{H}), 7.20(\mathrm{t}, J=7.3 \mathrm{~Hz}, 1 \mathrm{H} ; \mathrm{Ph}-\mathrm{H}), 7.12(\mathrm{~d}, J=7.2$ $\mathrm{Hz}, 2 \mathrm{H} ; \mathrm{Ph}-\mathrm{H}), 6.02$ (t, $J=4.2 \mathrm{~Hz}, 1 \mathrm{H}$; H-alkene), $4.16-4.04\left(\mathrm{~m}, 2 \mathrm{H} ; \mathrm{CH}_{2} \mathrm{O}\right), 2.80-2.67(\mathrm{~m}, 1 \mathrm{H} ; \mathrm{CH}$ cyclopropane), $2.62-2.51$ (m, 1H; $\mathrm{CH}$ cyclopropane), 2.22 (dd, $J=6.3,10.7 \mathrm{~Hz}, 2 \mathrm{H} ; \mathrm{CH}_{2}$ dihydropyran), $1.93-1.80$ (m, 2H; $\mathrm{CH}_{2}$ dihydropyran), 1.75 (ddd, $J=4.1,5.2,9.2 \mathrm{~Hz}, 1 \mathrm{H} ; \mathrm{CH}_{2}$ cyclopropane), 1.42 (ddd, $J$ $=4.0,6.6,8.1 \mathrm{~Hz}, 1 \mathrm{H} ; \mathrm{CH}_{2}$ cyclopropane $) .{ }^{13} \mathrm{C} \mathrm{NMR}\left(\mathrm{CDCl}_{3}, 100 \mathrm{MHz}\right) \delta 194.5,151.3,140.4,128.3,126.3$, 126.1, 109.7, 66.3, 29.7, 27.3, 21.44, 20.7, 19.6. IR v 2958 (s), 2928 (s), 1737 (w), 1666 (m), 1627 (s), 1510 (m), 1497 (m), 1458 (m), 1398 (m), 1341 (m), 1287 (s), 1261 (m), 1237 (m), 1205 (m), 1182 (s), 1159 (m), 1081 (s), 1061 (s), 1030 (s), 1007 (s), 919 (s), 795 (s), 756 (s), 699 (s). HRMS(ESI) calcd for $\mathrm{C}_{15} \mathrm{H}_{16} \mathrm{O}_{2}^{+}$ $(\mathrm{M}+\mathrm{H})$ 251.1042, found 251.1037.

(E)- $N$-Methoxy- $N$-methyl-3-(3,4-dimethoxyphenyl)-acrylamide (24) and $N$-methoxy- $N$-methyl-1-[2(3,4-dimethoxyphenyl)-cyclopropan-1-yl]-formamide (25)

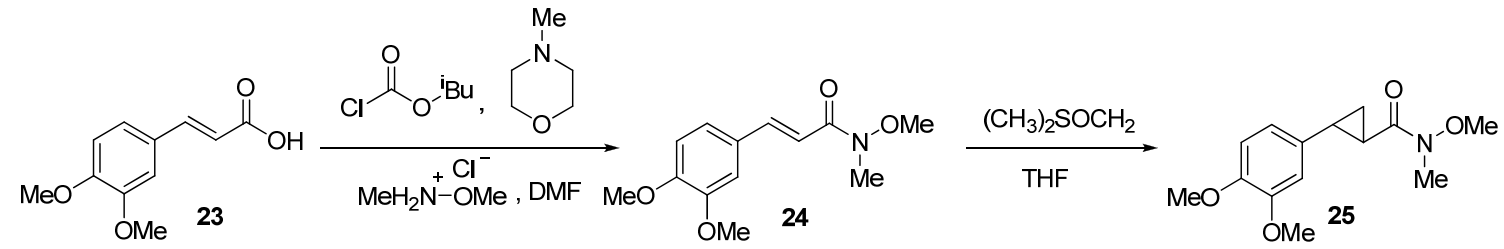

Following general procedure 1, the acid 23 (1.74 g, $8.36 \mathrm{mmol}, 1.00$ equiv) gave the Weinreb amide 24 which was used directly without purification. Using general procedure 3 , a solution of ylide $(16.5 \mathrm{~mL}, 8.91$ mmol, $1.20 \mathrm{eq})$ was added to a solution of amide 24 (1.85 g, $7.36 \mathrm{mmol}, 1.00$ equiv) in THF (75 mL). The reaction was stirred at room temperature during $3 \mathrm{~h}$ then quenched. Purification by flash column chromatography (PET/ AcOEt, 3/2) afforded 25 (918 mg, $3.45 \mathrm{mmol}, 47 \%)$ over 2 steps as colorless oil. $R_{f}$ 0.30 (PET/AcOEt 7:3, Anisaldehyde). ${ }^{1} \mathrm{H}$ NMR $\left(\mathrm{CDCl}_{3}, 400 \mathrm{MHz}\right) \delta 6.79$ (d, J = $\left.8.2 \mathrm{~Hz}, 1 \mathrm{H} ; \mathrm{Ar}-\mathrm{H}\right), 6.73-$ 6.64 (m, 2H; Ar-H), 3.87 (s, 3H; $\left.\mathrm{OCH}_{3}\right), 3.85$ (s, 3H; $\left.\mathrm{OCH}_{3}\right), 3.70\left(\mathrm{~s}, 3 \mathrm{H} ; \mathrm{OCH}_{3}\right), 3.24\left(\mathrm{~s}, 3 \mathrm{H} ; \mathrm{NCH}_{3}\right), 2.55$ - 2.41 (m, 1H; CH cyclopropane), 2.33 (s, $1 \mathrm{H} ; \mathrm{CH}$ cyclopropane), 1.59 (dt, $J=4.8,9.3 \mathrm{~Hz}, 1 \mathrm{H} ; \mathrm{CH}_{2}$ cyclopropane), 1.27 (ddd, $J=5.7,8.8,17.5 \mathrm{~Hz}, 1 \mathrm{H} ; \mathrm{CH}_{2}$ cyclopropane). ${ }^{13} \mathrm{C} \mathrm{NMR}\left(\mathrm{CDCl}_{3}, 100 \mathrm{MHz}\right) \delta$ 173.0, 148.8, 147.5, 133.2, 117.9, 111.2, 110.2, 61.6, 55.8, 55.7, 32.4, 25.6, 21.3, 15.9. IR v $3006(\mathrm{w}), 2963$ (w), $2938(\mathrm{w}), 2837(\mathrm{w}), 1645(\mathrm{w}), 1518(\mathrm{~m}), 1464(\mathrm{~m}), 1441(\mathrm{w}), 1420(\mathrm{w}), 1392(\mathrm{w}), 1254(\mathrm{~m}), 1235(\mathrm{~m})$, $1141(\mathrm{~m}), 1028(\mathrm{~m}), 1004(\mathrm{w}), 907$ (s), 805 (w), 726 (s), $648(\mathrm{~m})$. HRMS(ESI) calcd for $\mathrm{C}_{14} \mathrm{H}_{19} \mathrm{NO}_{4}{ }^{+}(\mathrm{M}+\mathrm{H})$ 266.1387, found 266.1385 .

${ }^{5}$ Liang, G. X.; Gradl, S. N.; Trauner, D. Org. Lett. 2003, 5, 4931-4934. 


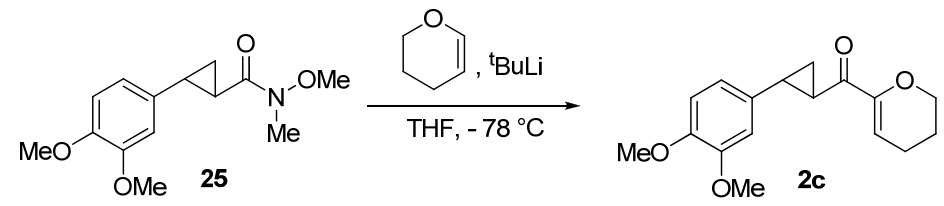

Following general procedure 4, dihydropyran (344 $\mu \mathrm{L}, 3.77 \mathrm{mmol}, 2.20$ equiv) and amide 25 (450 mg, $1.71 \mathrm{mmol}, 1.00$ equiv) were reacted together. The deprotonation time was $45 \mathrm{~min}$ at $0^{\circ} \mathrm{C}$ and the reaction was quenched after $45 \mathrm{~min}$. Purification by flash chromatography (PET/AcOEt, 7:3) afforded 2c (158 mg, $0.550 \mathrm{mmol}, 32 \%)$ as colorless oil. $R_{f} 0.40$ (PET/AcOEt 7:3, Anisaldehyde). ${ }^{1} \mathrm{H}$ NMR $\left(\mathrm{CDCl}_{3}, 400 \mathrm{MHz}\right) \delta$ $6.79(\mathrm{~d}, J=8.8 \mathrm{~Hz}, 1 \mathrm{H}$; Ar-H), $6.67(\mathrm{~d}, J=6.2 \mathrm{~Hz}, 2 \mathrm{H}$; Ar-H), 6.02 (t, $J=4.2 \mathrm{~Hz}, 1 \mathrm{H}$; alkene-H), $4.17-$ 4.07 (m, 2H; $\left.\mathrm{CH}_{2} \mathrm{O}\right), 3.87$ (s, 3H; $\left.\mathrm{OCH}_{3}\right), 3.85\left(\mathrm{~s}, 3 \mathrm{H} ; \mathrm{OCH}_{3}\right), 2.70-2.61$ (m, 1H; cyclopropane $\left.\mathrm{CH}\right), 2.54$ (ddd, $J=4.1,6.7,10.6 \mathrm{~Hz}, 1 \mathrm{H}$; cyclopropane $\mathrm{CH}), 2.22\left(\mathrm{dd}, J=6.3,10.7 \mathrm{~Hz}, 2 \mathrm{H}\right.$; dihydropyran $\left.\mathrm{CH}_{2}\right), 1.92$ - $1.81\left(\mathrm{~m}, 2 \mathrm{H}\right.$; dihydropyran $\left.\mathrm{CH}_{2}\right), 1.71\left(\mathrm{ddd}, J=4.2,5.1,9.1 \mathrm{~Hz}, 1 \mathrm{H}\right.$; cyclopropane $\left.\mathrm{CH}_{2}\right), 1.39(\mathrm{ddd}, J=$ 4.0, 6.7, 8.1 Hz, $1 \mathrm{H}$; cyclopropane $\left.\mathrm{CH}_{2}\right) .{ }^{13} \mathrm{C} \mathrm{NMR}\left(\mathrm{CDCl}_{3}, 100 \mathrm{MHz}\right) \delta 194.7,151.4,148.8,147.7,133.1$, $117.9,111.2,110.0,109.6,66.3,55.9,55.8,29.6,27.5,21.5,20.7,19.2$. IR v $3002(\mathrm{w}), 2935(\mathrm{w}), 2836(\mathrm{w})$, $2252(\mathrm{w}), 1663(\mathrm{w}), 1625(\mathrm{~m}), 1590(\mathrm{w}), 1518(\mathrm{~s}), 1464(\mathrm{~m}), 1439(\mathrm{w}), 1389(\mathrm{~m}), 1287(\mathrm{~m}), 1255(\mathrm{~m}), 1235$ (s), 1203 (m), 1181 (m), 1141 (m), 1091 (m), 1062 (m), 1027 (s), 962 (w), 913 (s), 806 (m), 729 (s). HRMS(ESI) calcd for $\mathrm{C}_{17} \mathrm{H}_{20} \mathrm{O}_{4}{ }^{+}(\mathrm{M}+\mathrm{H}) 289.1424$, found 289.1434 .

\section{(E)-2,4-Dimetoxy-cis-cinnamic acid (27)}
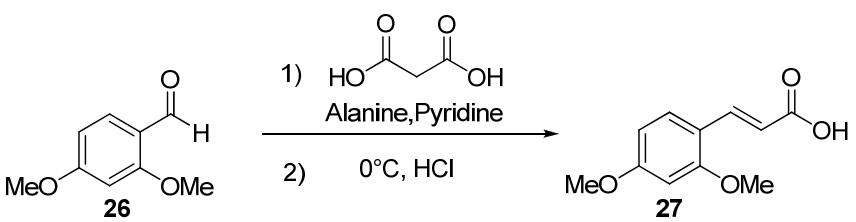

Following a reported procedure, ${ }^{6}$ a solution of aldehyde $27(11.0 \mathrm{~g}, 66.7 \mathrm{mmol}, 1.00$ equiv), malonic acid (17.5 g, $168 \mathrm{mmol}, 2.50$ equiv) and $\beta$-alanine ( $1.0 \mathrm{~g}, 89 \mathrm{mmol}, 0.20$ equiv) in pyridine $(3 \mathrm{~mL})$ was stirred under reflux for $90 \mathrm{~min}$. After cooling to RT, the flask was transferred in an ice bath and a concentrated solution of $\mathrm{HCl}(8 \mathrm{~mL})$ was added dropwise. The precipitate was filtered, washed with cold water $(2 \times 10 \mathrm{~mL})$ and dried without further purification to give 27 as light yellow solid (12.5 g, 60.0 mmol, 90\%). ${ }^{1} \mathrm{H}$ NMR $\left(\right.$ DMSO-d $\left._{6}, 400 \mathrm{MHz}\right) \delta 12.11(\mathrm{~s}, 1 \mathrm{H} ; \mathrm{OH}), 7.75$ (d, $\left.J=16.1 \mathrm{~Hz}, 1 \mathrm{H} ; \mathrm{CH}-\mathrm{Ar}\right), 7.61$ (d, $J=8.6 \mathrm{~Hz}, 1 \mathrm{H}$; Ar$\mathrm{H}), 6.64-6.54$ (m, 2H; Ar-H), 6.37 (d, $J=16.1 \mathrm{~Hz}, 1 \mathrm{H}$; CHCO), 3.86 (s, 3H; $\left.\mathrm{OCH}_{3}\right), 3.81$ (s, 3H; $\mathrm{OCH}_{3}$ ). ${ }^{1} \mathrm{H}$ NMR spectra corresponded to the literature values. ${ }^{7}$

(E)- $N$-Methoxy- $N$-methyl-3-(2,4-dimethoxyphenyl)-acrylamide (28) and $N$-methoxy- $N$-methyl-1-[2(2,4-dimethoxyphenyl)-cyclopropan-1-yl]-formamide (9)

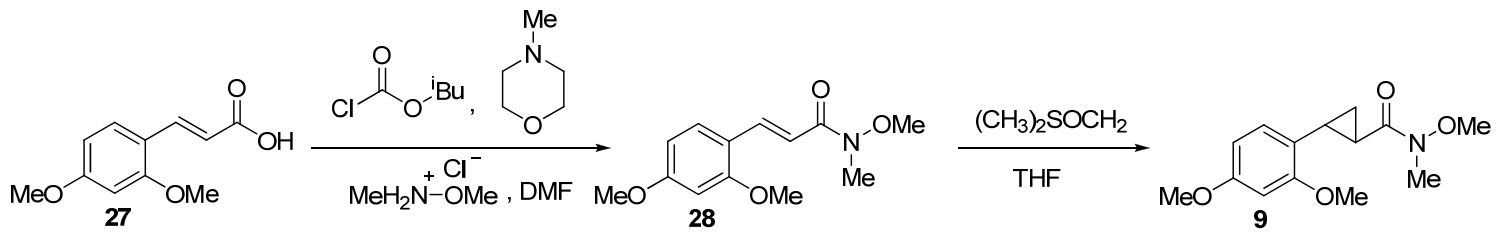

\footnotetext{
${ }^{6}$ Stabile, R. G.; Dicks, A. R. J. Chem. Educ. 2004, 81, 1488-1491.

${ }^{7}$ Luadthong, C.; Tachaprutinun, A.; Wanichwecharungruang, S. P. Eur. Polym. J. 2008, 44, 1285-1295.
} 
Following general procedure 1, the acid 27 (12.47 g, $59.89 \mathrm{mmol}, 1.000$ equiv) gave the Weinreb amide 28 which was used directly without purification. Using general procedure 3, a solution of ylide $(53.4 \mathrm{~mL}$, $28.8 \mathrm{mmol}, 1.20 \mathrm{eq})$ was added to a solution of amide $\mathbf{2 8}(6.00 \mathrm{~g}, 23.9 \mathrm{mmol}, 1.00$ equiv) in THF $(240 \mathrm{~mL})$. The reaction was stirred at RT overnight then quenched. Purification by flash column chromatography (PET/AcOEt, 3:2) gave 9 (3.93 g, $14.8 \mathrm{mmol}, 62 \%)$ over 2 steps as white solid. $R_{f} 0.35$ (PET/AcOEt 3:2, Anisaldehyde). Mp $58-59{ }^{\circ} \mathrm{C} .{ }^{1} \mathrm{H}$ NMR $\left(\mathrm{CDCl}_{3}, 400 \mathrm{MHz}\right) \delta 6.88(\mathrm{~d}, J=8.3 \mathrm{~Hz}, 1 \mathrm{H} ; \mathrm{Ar}-\mathrm{H}), 6.46-6.37$ $(\mathrm{m}, 2 \mathrm{H} ; \mathrm{Ar}-\mathrm{H}), 3.80\left(\mathrm{~s}, 3 \mathrm{H} ; \mathrm{OCH}_{3}\right), 3.79\left(\mathrm{~s}, 3 \mathrm{H} ; \mathrm{OCH}_{3}\right), 3.24\left(\mathrm{~s}, 3 \mathrm{H} ; \mathrm{NCH}_{3}\right), 2.64-2.51(\mathrm{~m}, 1 \mathrm{H} ; \mathrm{CH}$ cyclopropane), 2.25 (s, 1H; CH cyclopropane), 1.57 - 1.49 (m, 1H; $\mathrm{CH}_{2}$ cyclopropane), 1.30 - 1.20 (m, 1H; $\mathrm{CH}_{2}$ cyclopropane). ${ }^{13} \mathrm{C} \mathrm{NMR}\left(\mathrm{CDCl}_{3}, 100 \mathrm{MHz}\right) \delta 173.6,159.4,159.2,126.6,121.2,103.7,98.3,61.3$, 55.2, 55.2, 32.5, 20.7, 19.8, 14.3. IR v $3002(\mathrm{w}), 2960(\mathrm{w}), 2938(\mathrm{w}), 2837(\mathrm{w}), 1650$ (s), $1614(\mathrm{~m}), 1585$ (m), 1510 (s), 1461 (s), 1438 (s), 1417 (s), 1394 (m), 1364 (m), 1321 (w), 1289 (s), 1263 (m), 1208 (s), 1176 (s), 1159 (s), 1155 (s), 1127 (s), 1096 (m), 1033 (s), 933 (m), 920 (m), 874 (w), 834 (s), 800 (w), 775 (w), 729 (w). HRMS(ESI) calcd for $\mathrm{C}_{14} \mathrm{H}_{19} \mathrm{NO}_{4}{ }^{+}(\mathrm{M}+\mathrm{H}) 266.1387$, found 266.1381.

\section{2-[2-(2,4-Dimethoxyphenyl)-1-cyclopropanecarbonyl]-5,6-dihydro-4H-pyran (2d)}

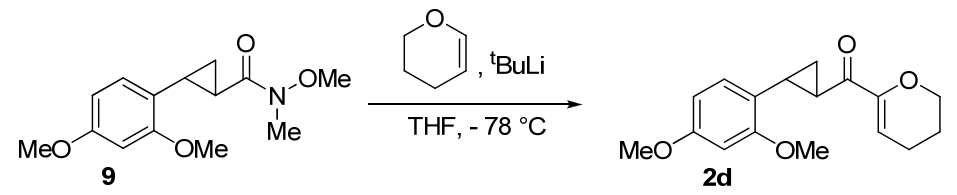

Following general procedure 4, a solution of amide 9 (400 $\mathrm{mg}, 1.51 \mathrm{mmol}, 1.00$ equiv) was added to a solution of dihydropyran $\left(345 \mu \mathrm{L}, 3.77 \mathrm{mmol}, 2.50\right.$ equiv). The deprotonation time was $30 \mathrm{~min}$ at $0^{\circ} \mathrm{C}$ and the reaction was quenched after $2 \mathrm{~h}$ and $15 \mathrm{mins}$ to give $2 \mathrm{~d}(305 \mathrm{mg}, 1.01 \mathrm{mmol}, 70 \%)$ as yellow oil after purification via flash chromatography (PET/AcOEt, 7:3). $R_{f} 0.55$ (PET/ AcOEt 7:3, Anisaldehyde). ${ }^{1} \mathrm{H}$ NMR $\left(\mathrm{CDCl}_{3}, 400 \mathrm{MHz}\right) \delta 6.87(\mathrm{~d}, J=8.2 \mathrm{~Hz}, 1 \mathrm{H} ; \mathrm{Ar}-\mathrm{H}), 6.41(\mathrm{dd}, J=2.3,10.6 \mathrm{~Hz}, 2 \mathrm{H} ; \mathrm{Ar}-\mathrm{H}), 6.02(\mathrm{t}, J=4.2$ $\mathrm{Hz}, 1 \mathrm{H}$; alkene-H), $4.13-4.08\left(\mathrm{~m}, 2 \mathrm{H} ; \mathrm{CH}_{2} \mathrm{O}\right), 3.79\left(\mathrm{~s}, 3 \mathrm{H} ; \mathrm{OCH}_{3}\right), 3.78\left(\mathrm{~s}, 3 \mathrm{H} ; \mathrm{OCH}_{3}\right), 2.74-2.62(\mathrm{~m}$, $1 \mathrm{H}$; cyclopropane $\mathrm{CH}), 2.57(\mathrm{dt}, J=4.8,9.3 \mathrm{~Hz}, 1 \mathrm{H}$; cyclopropane $\mathrm{CH}), 2.22(\mathrm{dd}, J=6.3,10.7 \mathrm{~Hz}, 2 \mathrm{H}$; dihydropyran $\left.\mathrm{CH}_{2}\right), 1.92-1.81\left(\mathrm{~m}, 2 \mathrm{H}\right.$; dihydropyran $\left.\mathrm{CH}_{2}\right), 1.71-1.63\left(\mathrm{~m}, 1 \mathrm{H}\right.$; cyclopropane $\left.\mathrm{CH}_{2}\right), 1.36$ (td, $J=3.8,7.6 \mathrm{~Hz}, 1 \mathrm{H}$; cyclopropane $\left.\mathrm{CH}_{2}\right) .{ }^{13} \mathrm{C} \mathrm{NMR}\left(\mathrm{CDCl}_{3}, 100 \mathrm{MHz}\right) \delta 195.2,159.4,159.2,151.5$, 126.5, 121.4, 109.4, 103.8, 98.3, 66.23, 55.3, 25.9, 24.7, 21.5, 20.7, 17.8. IR v 3010 (w), $2957(w), 2936$ (w), $2837(\mathrm{w}), 1681(\mathrm{~m}), 1666(\mathrm{~m}), 1625(\mathrm{~s}), 1586(\mathrm{~m}), 1510$ (m), 1464 (m), 1436 (m), 1397 (m), 1333 (m), 1289 (s), 1265 (w), 1237 (w), 1209 (s), 1182 (m), 1160 (m), 1124 (w), 1092 (w), 1063 (s), 1034 (s), 1002 (w), $954(\mathrm{w}), 918(\mathrm{~m}), 837(\mathrm{w})$. HRMS(ESI) calcd for $\mathrm{C}_{17} \mathrm{H}_{20} \mathrm{O}_{4}{ }^{+}(\mathrm{M}+\mathrm{H}) 289.1434$, found 289.1412.

(E)- $N$-Methoxy- $N$-methyl-3-(2-furanyl)-acrylamide (30) and $N$-methoxy- $N$-methyl-1-[2-(2-furanyl)cyclopropan-1-yl]-formamide (31)

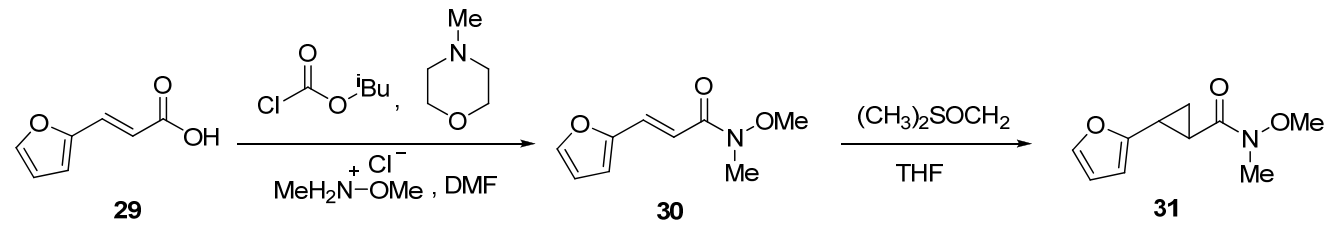

Following general procedure 1, the acid 29 (1.55 g, $11.2 \mathrm{mmol}, 1.00$ equiv) gave the Weinreb amide 30 which was used directly without purification. Using the general procedure 3, a solution of ylide (10 $\mathrm{mL}, 5.4$ mmol, $1.2 \mathrm{eq})$ was added to a solution of amide 31 (810 g, $4.47 \mathrm{mmol}, 1.00$ equiv) in THF (46 mL). The reaction was warmed to $40^{\circ} \mathrm{C}$ and stirred during $3 \mathrm{~h}$ before quenching. Purification by flash column chromatography (PET/AcOEt, 7:3) gave 28 (436 mg, $2.24 \mathrm{mmol}, 50 \%)$ over two steps as colorless oil. $R_{f}$ 0.50 (PET/AcOEt 7:3, Anisaldehyde). ${ }^{1} \mathrm{H}$ NMR $\left(\mathrm{CDCl}_{3}, 400 \mathrm{MHz}\right) \delta 7.27-7.25(\mathrm{~m}, 1 \mathrm{H}$; furan-H), 6.28 
$\left(\mathrm{dd}, J=1.9,3.0 \mathrm{~Hz}, 1 \mathrm{H}\right.$; furan-H), $6.07\left(\mathrm{~d}, J=3.1 \mathrm{~Hz}, 1 \mathrm{H}\right.$; furan-H), $3.73\left(\mathrm{~s}, 3 \mathrm{H} ; \mathrm{OCH}_{3}\right), 3.23(\mathrm{~s}, 3 \mathrm{H}$; $\left.\mathrm{NCH}_{3}\right), 2.58-2.40$ (m, 2H; 2xCH cyclopropane), $1.59-1.50\left(\mathrm{~m}, 1 \mathrm{H} ; \mathrm{CH}_{2}\right.$ cyclopropane), $1.44-1.32$ (m, $1 \mathrm{H} ; \mathrm{CH}_{2}$ cyclopropane). ${ }^{13} \mathrm{C} \mathrm{NMR}\left(\mathrm{CDCl}_{3}, 100 \mathrm{MHz}\right) \delta 172.6,153.7,140.8,110.3,105.0,61.5,32.4,19.2$, 18.9, 14.0. IR v $3522(\mathrm{w}), 2938(\mathrm{w}), 1648(\mathrm{~s}), 1509(\mathrm{~m}), 1462(\mathrm{~m}), 1440(\mathrm{~s}), 1423(\mathrm{~s}), 1389(\mathrm{~m}), 1354$ (m), $1176(\mathrm{~m}), 1148(\mathrm{~m}), 1115(\mathrm{~m}), 1098(\mathrm{~m}), 995$ (s), $952(\mathrm{~m}), 914(\mathrm{~m}), 801$ (m), 733 (s). HRMS(ESI) calcd for $\mathrm{C}_{10} \mathrm{H}_{13} \mathrm{NO}_{3}{ }^{+}(\mathrm{M}+\mathrm{H})$ 196.0968, found 196.0975.

\section{2-[2-(Furan-2-yl)-1-cyclopropanecarbonyl]-5,6-dihydro-4H-pyran (2e)}

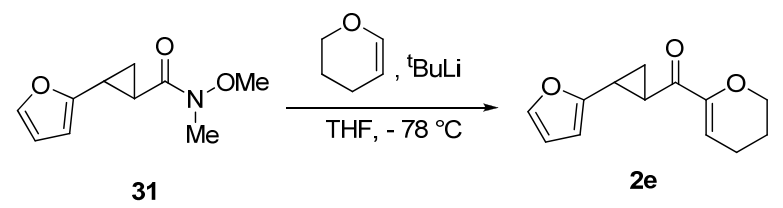

Following general procedure 4, dihydropyran (400 $\mu \mathrm{L}, 4.40 \mathrm{mmol}, 2.00$ equiv) and amide 31 (430 mg, $2.20 \mathrm{mmol}, 1.00$ equiv) were reacted together. The deprotonation time was $30 \mathrm{~min}$ at $0^{\circ} \mathrm{C}$ and the reaction was quenched after $1 \mathrm{~h}$. Purification by flash chromatography (PET/AcOEt, 9:1) afforded 2e (300 mg, 1.38 mmol, $63 \%$ ) as a yellow oil. $R_{f} 0.25$ (PET/AcOEt 10:1, Anisaldehyde). ${ }^{1} \mathrm{H}$ NMR $\left(\mathrm{CDCl}_{3}, 400 \mathrm{MHz}\right) \delta=$ 7.26 (m, 1H; Ar-H), 6.29 (s, 1H; Ar-H), 6.08 (s, 1H; Ar-H), 6.04 (t, J= 3.9 Hz, 1H; alkene-H), $4.16-4.07$ (m, $2 \mathrm{H} ; \mathrm{CH}_{2} \mathrm{O}$ ), $2.89-2.73(\mathrm{~m}, 1 \mathrm{H}$; cyclopropane $\mathrm{CH}), 2.57$ (t, J=9.6 Hz, 1H; cyclopropane $\left.\mathrm{CH}\right), 2.23$ (dd, $J=5.8,10.6 \mathrm{~Hz}, 2 \mathrm{H}$; dihydropyran $\left.\mathrm{CH}_{2}\right), 1.87\left(\mathrm{dd}, J=5.6,10.8 \mathrm{~Hz}, 2 \mathrm{H}\right.$; dihydropyran $\left.\mathrm{CH}_{2}\right), 1.67-1.58(\mathrm{~m}$, $1 \mathrm{H}$; cyclopropane $\left.\mathrm{CH}_{2}\right), 1.47\left(\mathrm{t}, J=9.0 \mathrm{~Hz}, 1 \mathrm{H}\right.$; cyclopropane $\left.\mathrm{CH}_{2}\right) \cdot{ }^{13} \mathrm{C} \mathrm{NMR}\left(\mathrm{CDCl}_{3}, 100 \mathrm{MHz}\right) \delta 193.6$, 153.4, 150.9, 140.6, 110.1, 109.7, 104.9, 65.9, 24.6, 22.1, 21.1, 20.4, 16.8. IR v 3118 (w), 2932 (w), 2873 $(\mathrm{w}), 1683(\mathrm{~m}), 1668(\mathrm{~m}), 1626(\mathrm{~s}), 1509(\mathrm{w}), 1445(\mathrm{w}), 1431(\mathrm{w}), 1385(\mathrm{~m}), 1350(\mathrm{w}), 1314(\mathrm{w}), 1287(\mathrm{~m})$, 1246 (m), 1236 (m), 1202 (m), 1180 (m), 1149 (w), 1091 (m), 1061 (s), 1034 (m), 1008 (m), 991 (m), 955 (w), $916(\mathrm{~s}), 886(\mathrm{w}), 797(\mathrm{~m}), 789(\mathrm{~m}), 730$ (s). HRMS(ESI) calcd for $\mathrm{C}_{13} \mathrm{H}_{14} \mathrm{O}_{3}{ }^{+}(\mathrm{M}+\mathrm{H})$ 219.1016, found 219.1011.

\section{(E)-3-(4-Methoxyphenyl)-2-methyl-acrylic acid (32)}

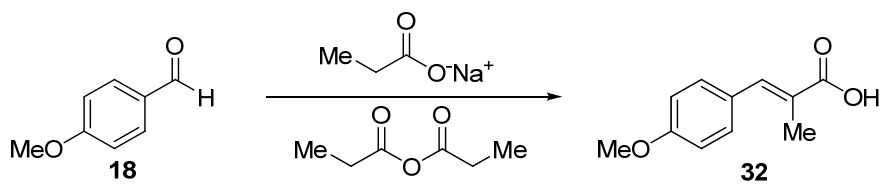

Following a reported procedure, ${ }^{8}$ a mixture of anisaldehyde 18 (10 mL, $82 \mathrm{mmol}, 1.0$ equiv), propionic anhydride (19 mL, $0.16 \mathrm{~mol}, 2.0$ equiv) and sodium propionate $(7.9 \mathrm{~g}, 82 \mathrm{mmol}, 1.0$ equiv) was heated at $150{ }^{\circ} \mathrm{C}$ for $12 \mathrm{~h}$. After cooling to $23{ }^{\circ} \mathrm{C}, 4 \mathrm{M} \mathrm{NaOH}$ solution $(60 \mathrm{~mL})$ was added and the mixture was washed with $\mathrm{Et}_{2} \mathrm{O}(2 \times 20 \mathrm{~mL})$. The water layer was acidified to $\mathrm{pH}=1$ with conc. $\mathrm{HCl}$ and the precipitated colorless solid was washed with water and dried in high vacuo to give 32 as a colorless solid $(10.0 \mathrm{~g}, 52.1 \mathrm{mmol}$, 63\%). Mp 154-157 ${ }^{\circ} \mathrm{C}, \mathrm{Lit}^{8}$ : $152-155^{\circ} \mathrm{C} .{ }^{1} \mathrm{H} \mathrm{NMR}\left(\mathrm{CDCl}_{3}, 400 \mathrm{MHz}\right) \delta 7.79$ (br s, $1 \mathrm{H}$; alkene-H), 7.43 (d, $J=8.6 \mathrm{~Hz}, 2 \mathrm{H} ; \mathrm{Ar}-\mathrm{H}), 6.95(\mathrm{~d}, J=9.0 \mathrm{~Hz}, 2 \mathrm{H} ; \mathrm{Ar}-\mathrm{H}), 3.85\left(\mathrm{~s}, 3 \mathrm{H} ; \mathrm{OCH}_{3}\right), 2.16(\mathrm{~d}, J=1.6 \mathrm{~Hz}, 3 \mathrm{H}$; $\left.\mathrm{CHCH}_{3}\right) .{ }^{13} \mathrm{C} \mathrm{NMR}\left(\mathrm{CDCl}_{3}, 75 \mathrm{MHz}\right) \delta 174.5,160.0,140.8,131.7,128.2,125.2,113.9,55.3,13.7$. IR $v$ $2939(\mathrm{w}), 2838(\mathrm{w}), 2634(\mathrm{w}), 2512(\mathrm{w}), 1661(\mathrm{~m}), 1600(\mathrm{~m}), 1569(\mathrm{~m}), 1511(\mathrm{~m}), 1424(\mathrm{~m}), 1320(\mathrm{~m})$, 1275 (s), 1253 (s), 1180 (m), 1130 (m), 1030 (m), 912 (s), 829 (s), 808 (s), 746 (s), 693 (m), 652 (w). ${ }^{1} \mathrm{H}$ NMR and IR spectra corresponded to the literature values. ${ }^{8}$

\footnotetext{
${ }^{8}$ Hagishita, S.; Yamada, M.; Shirahase, K.; Okada, T.; Murakami, Y.; Ito, Y.; Matsuura, T.; Wada, M.; Kato, T.; Ueno, M.; Chikazawa, Y.; Yamada, K.; Ono, T.; Teshirogi, I.; Ohtani, M. J. Med. Chem. 1996, 39, 3636-3658.
} 
(E)- $N$-Methoxy- $N$-methyl-3-(4-methoxyphenyl)-2-methyl-acrylamide (33)

and $N$-methoxy- $N$ methyl-1-[2-(4-methoxyphenyl)-cyclopropan-1-methyl-1-yl]-formamide (34)

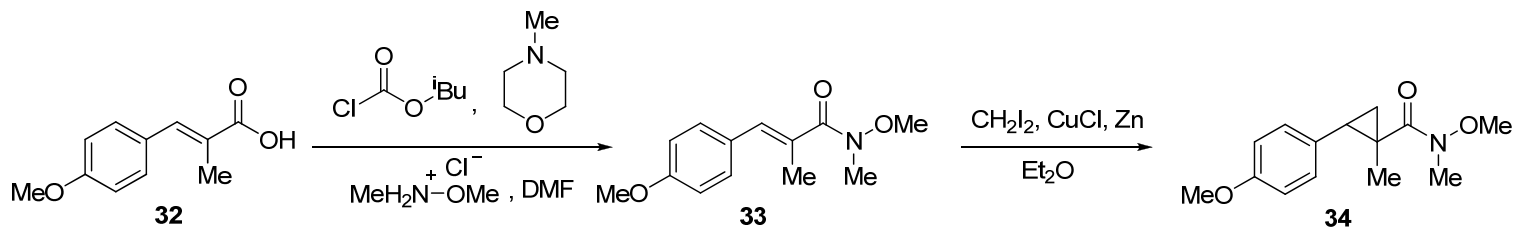

Following general procedure 1, the acid 32 (3.00 g, $15.6 \mathrm{mmol}, 1.00$ equiv) gave the Weinreb amide 33 which was used directly without purification. A suspension of copper(I) chloride (1.5 g, 15 mmol, 5.0 equiv, purified via precipitation from a solution in conc. $\mathrm{HCl}$, washed with water, $\mathrm{EtOH} \mathrm{Et}_{2} \mathrm{O}$ and dried in $\mathrm{HV}$ ) and Zinc dust (activated with $1 \% \mathrm{HCl}$, washed with water, $\mathrm{EtOH}, \mathrm{Et}_{2} \mathrm{O}$ and dried in $\mathrm{HV}, 1.0 \mathrm{~g}, 15 \mathrm{mmol}, 5.0$ equiv) in $\mathrm{Et}_{2} \mathrm{O}(7 \mathrm{~mL})$ was refluxed for $30 \mathrm{~min}$. The reaction mixture was cooled to $23{ }^{\circ} \mathrm{C}$ and a solution of Weinreb amide 33 (0.71 g, $3.0 \mathrm{mmol}, 1.0$ equiv) in $\mathrm{Et}_{2} \mathrm{O}(2 \mathrm{~mL})$ was added. After refluxing for $72 \mathrm{~h}$, the reaction mixture was cooled to $23{ }^{\circ} \mathrm{C}$ and quenched with sat. $\mathrm{NH}_{4} \mathrm{Cl}(5 \mathrm{~mL})$. Water $(5 \mathrm{~mL})$ was added and the reaction mixture was extracted with $\mathrm{Et}_{2} \mathrm{O}(3 \times 10 \mathrm{~mL})$. The combined organic layers were washed with brine $(10 \mathrm{~mL})$, dried over $\mathrm{MgSO}_{4}$ and the solvent was removed under reduced pressure. The crude product was purified by flash column chromatography (PET/AcOEt 5:1-4:1) to yield cyclopropane 34 (0.15 g, 0.61 $\mathrm{mmol}, 20 \%, 61 \% \mathrm{brsm})$ as a colorless oil as well as recovered starting material 33 (0.47 g, $2.0 \mathrm{mmol}, 66 \%)$. $R_{f} 0.55$ (PET/AcOEt 1:1, Anisaldehyde). ${ }^{1} \mathrm{H}$ NMR $\left(\mathrm{CDCl}_{3}, 400 \mathrm{MHz}\right) \delta 7.15$ (dd, $J=9.0,0.6 \mathrm{~Hz}, 2 \mathrm{H}$; Ar$\mathrm{H}), 6.85\left(\mathrm{~d}, J=8.6 \mathrm{~Hz}, 2 \mathrm{H}\right.$; Ar-H), $3.80\left(\mathrm{~s}, 3 \mathrm{H} ; \mathrm{OCH}_{3}\right), 3.76\left(\mathrm{~s}, 3 \mathrm{H} ; \mathrm{OCH}_{3}\right), 3.25\left(\mathrm{~s}, 3 \mathrm{H}, \mathrm{NCH}_{3}\right), 2.44(\mathrm{dd}, J$ $=9.3,6.7 \mathrm{~Hz}, 1 \mathrm{H}$; cyclopropane $\mathrm{CH}), 1.59\left(\mathrm{~m}, 1 \mathrm{H}\right.$, cyclopropane $\left.\mathrm{CH}_{2}\right), 1.06\left(\mathrm{~s}, 3 \mathrm{H}\right.$, cyclopropane- $\left.\mathrm{CH}_{3}\right)$, $0.96\left(\mathrm{dd}, J=6.7,5.1 \mathrm{~Hz}, 1 \mathrm{H}\right.$, cyclopropane $\left.\mathrm{CH}_{2}\right) .{ }^{13} \mathrm{C} \mathrm{NMR}\left(\mathrm{CDCl}_{3}, 100 \mathrm{MHz}\right) \delta 174.9,158.1,130.0$, 129.3, 113.5, 60.8, 55.1, 33.8, 27.1, 26.7, 17.1, 16.1. IR $v 2934$ (w), 1646 (m), 1515 (s), 1463 (m), $1247(\mathrm{~s})$, $1176(\mathrm{~m}), 1029$ (m), 911 (s), $837(\mathrm{~m}), 730$ (s), 647 (w). HRMS(ESI) calcd for $\mathrm{C}_{14} \mathrm{H}_{19} \mathrm{NO}_{3}{ }^{+}(\mathrm{M}+\mathrm{Na})$ 272.1257, found 272.1259 .

\section{2-[2-(4-Methoxyphenyl)-1-methyl-1-cyclopropanecarbony]-5,6-dihydro-4H-pyran (2f)}

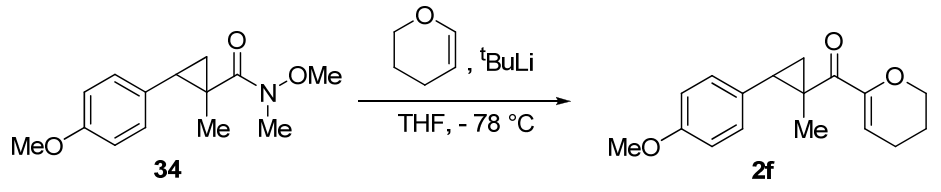

Following general procedure 4, dihydropyran (44 mL, $0.48 \mathrm{mmol}, 2.0$ equiv) and amide 34 (59 mg, 0.24 mmol, 1.0 equiv) were reacted together. The deprotonation time was $30 \mathrm{~min}$ at $0^{\circ} \mathrm{C}$ and the reaction was quenched after $30 \mathrm{~min}$. Purification by flash chromatography (PET/AcOEt 10:1-5:1) afforded 2 f (39 mg, $0.14 \mathrm{mmol}, 60 \%)$ as colorless oil. $R_{f} 0.70$ (PET/ AcOEt 2:1, Anisaldehyde). ${ }^{1} \mathrm{H}$ NMR $\left(\mathrm{CDCl}_{3}, 400 \mathrm{MHz}\right) \delta$ $7.13(\mathrm{~d}, J=8.0 \mathrm{~Hz}, 2 \mathrm{H} ; \mathrm{Ar}-\mathrm{H}), 6.85(\mathrm{~d}, J=8.6 \mathrm{~Hz}, 2 \mathrm{H} ; \mathrm{Ar}-\mathrm{H}), 5.80(\mathrm{t}, J=4.2 \mathrm{~Hz}, 1 \mathrm{H}$; alkene-H), 4.09 (m, $\left.2 \mathrm{H} ; \mathrm{CH}_{2} \mathrm{O}\right), 3.79\left(\mathrm{~s}, 3 \mathrm{H} ; \mathrm{OCH}_{3}\right), 2.50(\mathrm{dd}, J=9.0,6.7 \mathrm{~Hz}, 1 \mathrm{H}$; cyclopropane $\mathrm{CH}), 2.20(\mathrm{~m}, 2 \mathrm{H}$; dihydropyran $\left.\mathrm{CH}_{2}\right), 1.87$ (m, 2H; dihydropyran $\left.\mathrm{CH}_{2}\right), 1.82\left(\mathrm{dd}, \mathrm{J}=9.3,4.8 \mathrm{~Hz}, 1 \mathrm{H}\right.$; cyclopropane $\mathrm{CH}_{2}$ ),

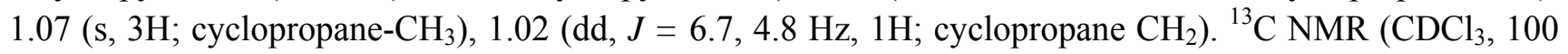
MHz) $\delta 198.3,158.2,151.5,130.1,129.1,113.5,109.2,65.9,55.2,31.8,30.4,21.6,20.5,18.2$, 15.9. IR $v$ $2956(\mathrm{w}), 2930(\mathrm{w}), 1669(\mathrm{w}), 1624(\mathrm{~m}), 1613(\mathrm{~m}), 1515(\mathrm{~s}), 1463(\mathrm{w}), 1444(\mathrm{w}), 1382(\mathrm{w}), 1290(\mathrm{~m}), 1247$ (s), $1173(\mathrm{~m}), 1061(\mathrm{~m}), 1036(\mathrm{~m}), 1003(\mathrm{~m}), 916(\mathrm{~s}), 836(\mathrm{~m}), 732(\mathrm{~s}), 648(\mathrm{w})$. HRMS(ESI) calcd for $\mathrm{C}_{17} \mathrm{H}_{20} \mathrm{O}_{3}^{+}(\mathrm{M}-\mathrm{H})$ 271.1329, found 271.1333.

\section{2-[2-(4-Methoxy-phenyl)-1-cyclopropanecarbonyl]-4,5-dihydro-furan (4a)}




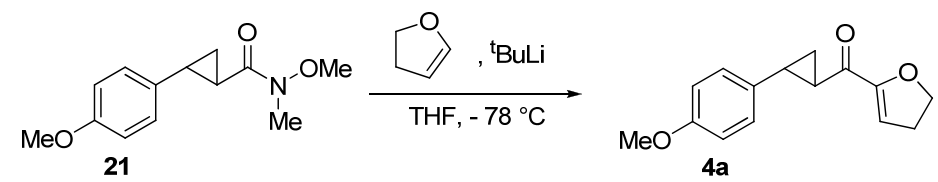

The reaction was carried out following general procedure 4 from dihydrofuran ( $353 \mu \mathrm{L}, 4.67 \mathrm{mmol}, 2.20$ equiv) and amide 21 (500 mg, $2.12 \mathrm{mmol}, 1.00$ equiv). The deprotonation time was $30 \mathrm{~min}$ at $0^{\circ} \mathrm{C}$ and the reaction was quenched after 1 hour. Purification by flash chromatography (PET/AcOEt, 7:3) afforded 4a (517 mg, $2.12 \mathrm{mmol}, 100 \%$ ) as colorless oil. $R_{f} 0.55$ (PET/AcOEt 7:3, Anisaldehyde). ${ }^{1} \mathrm{H} \mathrm{NMR}\left(\mathrm{CDCl}_{3}, 400\right.$ MHz) $\delta 7.05$ (d, $J=8.7 \mathrm{~Hz}, 2 \mathrm{H}$; Ar-H), 6.83 (d, $J=8.6 \mathrm{~Hz}, 2 \mathrm{H}$; Ar-H), 5.99 (t, $J=3.1 \mathrm{~Hz}, 1 \mathrm{H}$; alkene-H ), $4.49\left(\mathrm{t}, J=9.8 \mathrm{~Hz}, 2 \mathrm{H} ; \mathrm{CH}_{2} \mathrm{O}\right), 3.79\left(\mathrm{~s}, 3 \mathrm{H} ; \mathrm{OCH}_{3}\right), 2.84\left(\mathrm{td}, J=3.1,9.8 \mathrm{~Hz}, 2 \mathrm{H}\right.$; dihydrofuran $\left.\mathrm{CH}_{2}\right), 2.63$ - $2.54(\mathrm{~m}, 1 \mathrm{H}$; cyclopropane $\mathrm{CH}), 2.52-2.44(\mathrm{~m}, 1 \mathrm{H}$; cyclopropane $\mathrm{CH}), 1.79-1.72(\mathrm{~m}, 1 \mathrm{H}$; cyclopropane $\left.\mathrm{CH}_{2}\right), 1.44-1.37$ (m, 1H; cyclopropane $\left.\mathrm{CH}_{2}\right) .{ }^{13} \mathrm{C} \mathrm{NMR}\left(\mathrm{CDCl}_{3}, 100 \mathrm{MHz}\right) \delta 190.7,158.4,155.8,132.2$, 127.3, 113.9, 111.3, 70.3, 55.3, 30.7, 29.4, 29.4, 19.0. IR $\vee 3004(\mathrm{w}), 2936(\mathrm{w}), 2836(\mathrm{w}), 1649(\mathrm{~s}), 1614$ (m), 1516 (s), 1462 (m), 1442 (m), 1421 (m), 1394 (w), 1367 (w), 1303 (w), 1289 (w), 1248 (s), 1177 (m), $1122(\mathrm{~m}), 1097(\mathrm{~m}), 1035$ (m), 997 (m), 942 (w), $824(\mathrm{~m}), 809$ (m), 767 (w), 743 (w). HRMS(ESI) calcd for $\mathrm{C}_{15} \mathrm{H}_{16} \mathrm{O}_{5}^{+}(\mathrm{M}+\mathrm{H}) 245.1172$, found 245.1178.

\section{2-[2-(2,4-Methoxy-phenyl)-1-cyclopropanecarbonyl]-4,5-dihydro-furan (4b)}

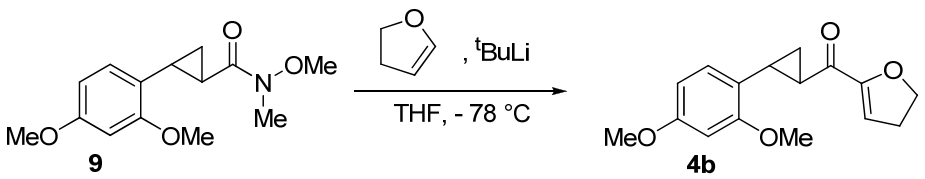

Amide 9 (400 mg, $1.51 \mathrm{mmol}, 1.00$ equiv) was added to dihydrofuran ( $285 \mu \mathrm{L}, 3.77 \mathrm{mmol}, 2.50$ equiv), following general procedure 4. The deprotonation time was $30 \mathrm{~min}$ at $0^{\circ} \mathrm{C}$ and the reaction was quenched after $45 \mathrm{~min}$ to give $\mathbf{4 b}(414 \mathrm{mg}, 1.51 \mathrm{mmol}, 100 \%)$ as white solid without further purification. $R_{f} 0.45$ (PET/AcOEt 7:3, Anisaldehyde). Mp 100-102 ${ }^{\circ} \mathrm{C} .{ }^{1} \mathrm{H} \mathrm{NMR}\left(\mathrm{CDCl}_{3}, 400 \mathrm{MHz}\right) \delta 6.87(\mathrm{~d}, J=8.2 \mathrm{~Hz}, 1 \mathrm{H}$; Ar-H), $6.41\left(\mathrm{dt}, J=2.3,8.3 \mathrm{~Hz}, 2 \mathrm{H}\right.$; Ar-H), 5.98 (t, $J=3.1 \mathrm{~Hz}, 1 \mathrm{H}$; alkene-H), $4.57-4.41\left(\mathrm{~m}, 2 \mathrm{H} ; \mathrm{CH}_{2} \mathrm{O}\right)$, $3.79\left(\mathrm{~s}, 3 \mathrm{H} ; \mathrm{OCH}_{3}\right), 3.78\left(\mathrm{~s}, 3 \mathrm{H} ; \mathrm{OCH}_{3}\right), 2.84\left(\mathrm{td}, J=3.1,9.8 \mathrm{~Hz}, 2 \mathrm{H}\right.$; dihydrofuran $\left.\mathrm{CH}_{2}\right), 2.70(\mathrm{ddd}, J=$ 4.2, 7.1, 9.0 Hz, 1H; cyclopropane $\mathrm{CH}), 2.41(\mathrm{dt}, J=4.9,8.1 \mathrm{~Hz}, 1 \mathrm{H}$; cyclopropane $\mathrm{CH}), 1.79-1.65(\mathrm{~m}$, $1 \mathrm{H}$; cyclopropane $\left.\mathrm{CH}_{2}\right), 1.41\left(\mathrm{td}, J=3.9,7.6 \mathrm{~Hz}, 1 \mathrm{H}\right.$; cyclopropane $\left.\mathrm{CH}_{2}\right) .{ }^{13} \mathrm{C} \mathrm{NMR}\left(\mathrm{CDCl}_{3}, 100 \mathrm{MHz}\right) \delta$ $191.2,159.6,159.3,156.0,126.7,121.0,111.0,103.8,98.4,70.2,55.3,30.7,28.0,25.0,17.5$. IR $v 3103(\mathrm{w})$, $3000(\mathrm{w}), 2959(\mathrm{w}), 2936(\mathrm{w}), 2836(\mathrm{w}), 1663(\mathrm{~m}), 1613(\mathrm{~s}), 1585(\mathrm{~m}), 1509$ (m), $1466(\mathrm{~m}), 1456(\mathrm{~m}), 1436$ (m), 1410 (m), 1342 (w), 1291 (m), 1264 (m), 1209 (s), 1175 (m), 1160 (m), 1060 (m), 1044 (s), 1013 (m), $940(\mathrm{~m}), 905(\mathrm{w}), 838(\mathrm{~m}), 799(\mathrm{w}), 729(\mathrm{w})$. HRMS(ESI) calcd for $\mathrm{C}_{16} \mathrm{H}_{18} \mathrm{O}_{4}{ }^{+}(\mathrm{M}+\mathrm{H})$ 275.1278, found 275.1240 .

\section{2-[2-(4-Methoxyphenyl)-1-cyclopropanecarbonyl]-1-methylindole (6)}

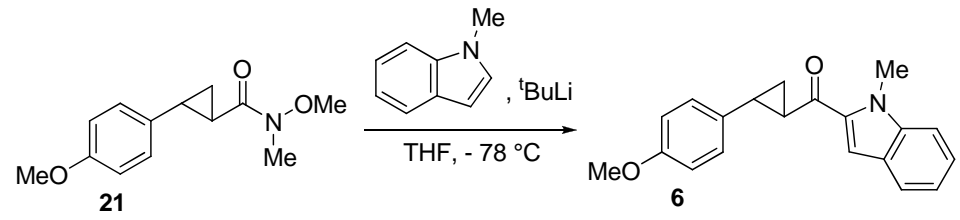

Following general procedure 4, $N$-methylindole $(192 \mu \mathrm{L}, 1.50 \mathrm{mmol}, 2.20$ equiv) was added to amide 21 (160 mg, $0.680 \mathrm{mmol}, 1.00$ equiv). The deprotonation time was $15 \mathrm{~min}$ at $0^{\circ} \mathrm{C}$ and the reaction was quenched after $1 \mathrm{~h}$. Purification by flash chromatography (PET/AcOEt, 7:3) afforded 8 (105 mg, 0.340 
mmol, 50\%) as white crystals. $R_{f} 0.80$ (PET/AcOEt 7:3, Anisaldehyde). Mp 103-105 ${ }^{\circ} \mathrm{C} .{ }^{1} \mathrm{H} \mathrm{NMR}\left(\mathrm{CDCl}_{3}\right.$, $400 \mathrm{MHz}) \delta 7.68$ (d, $J=8.0 \mathrm{~Hz}, 1 \mathrm{H}$; Ar-H), 7.41 - 7.35 (m, 3H; Ar-H), 7.19 - 7.09 (m, 3H; Ar-H), 6.86 (d, $J=8.7 \mathrm{~Hz}, 2 \mathrm{H} ; \mathrm{Ar}-\mathrm{H}), 4.10\left(\mathrm{~s}, 3 \mathrm{H} ; \mathrm{NCH}_{3}\right), 3.81\left(\mathrm{~s}, 3 \mathrm{H} ; \mathrm{OCH}_{3}\right), 2.91-2.78(\mathrm{~m}, 1 \mathrm{H}$; cyclopropane $\mathrm{CH})$, 2.65 (ddd, $J=4.1,6.7,10.4 \mathrm{~Hz}, 1 \mathrm{H}$; cyclopropane $\mathrm{CH}$ ), $1.91-1.80$ (m, 1H; cyclopropane $\mathrm{CH}_{2}$ ), 1.47 (ddd, $J=4.2,6.7,8.0 \mathrm{~Hz}, 1 \mathrm{H}$; cyclopropane $\left.\mathrm{CH}_{2}\right) .{ }^{13} \mathrm{C} \mathrm{NMR}\left(\mathrm{CDCl}_{3}, 100 \mathrm{MHz}\right) \delta 191.6,158.4,140.1,135.6$, 132.6, 127.5, 125.9, 122.8, 120.6, 114.0, 111.5, 110.3, 55.3, 32.2, 30.6, 28.7, 18.2. IR v $3002(\mathrm{w}), 2961(\mathrm{w})$, 2937 (w), 2835 (w), 1648 (s), 1614 (m), 1515 (s), 1466 (m), 1439 (w), 1428 (w), 1404 (m), 1380 (w), 1323 (w), $1293(\mathrm{w}), 1250(\mathrm{~s}), 1195(\mathrm{~m}), 1163(\mathrm{w}), 1152(\mathrm{w}), 1129(\mathrm{w}), 1032(\mathrm{~m}), 991(\mathrm{~m}), 913(\mathrm{w}), 824(\mathrm{w}), 748$ (m). HRMS(ESI) calcd for $\mathrm{C}_{20} \mathrm{H}_{19} \mathrm{NO}_{2}^{+}(\mathrm{M}+\mathrm{H}) 306.1489$, found 306.1475.

(E)-2-[2-(4-Methoxyphenyl)-1-ethylenecarbonyl]-benzofurane (36)

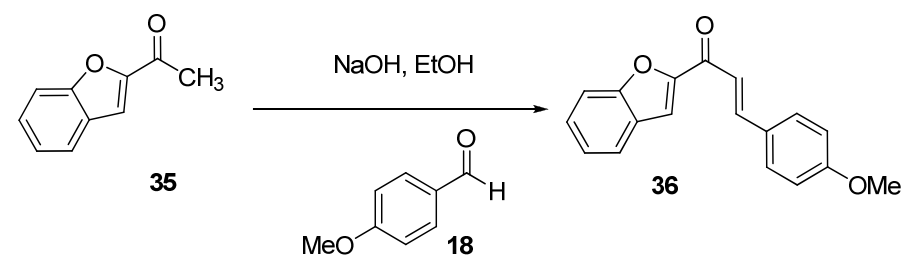

Following a slight modification of the reported procedure, ${ }^{3} \mathrm{NaOH}(4.4 \mathrm{~mL}, 2.5 \mathrm{~m})$ was added to a solution of 35 (2.50 g, $15.6 \mathrm{mmol}, 1.00$ equiv) in EtOH $(40 \mathrm{~mL})$ at RT. The reaction was stirred for $5 \mathrm{~min}$ then $p$-anisaldehyde 18 (1.90 mL, $15.6 \mathrm{mmol}, 1.00$ equiv) was added dropwise. The solution was quenched after 30 min with water $(20 \mathrm{~mL})$ and extracted with $\mathrm{Et}_{2} \mathrm{O}(40 \times 2 \mathrm{~mL})$. The organic layer was washed with brine, dried over $\mathrm{MgSO}_{4}$, concentrated and purified by flash column chromatography (PET/AcOEt 9:1) to yield 36 (1.74 g, $6.25 \mathrm{mmol}, 40 \%$ ) as yellow oil. $R_{f} 0.70$ (PET/AcOEt 7:3, Anisaldehyde). Mp 125-127 ${ }^{\circ} \mathrm{C}$. ${ }^{1} \mathrm{H}$ NMR $\left(\mathrm{CDCl}_{3}, 400 \mathrm{MHz}\right) \delta 7.93$ (d, $\left.J=15.7,1 \mathrm{H} ; \mathrm{CH}-\mathrm{Ar}\right), 7.73$ (d, $\left.J=7.8 \mathrm{~Hz}, 1 \mathrm{H} ; \mathrm{Ar}-\mathrm{H}\right), 7.66$ (d, $J=$ $8.7 \mathrm{~Hz}, 2 \mathrm{H}$; Ar-H), 7.63 (d, J = 6.7 Hz, 2H; Ar-H), 7.45 (m, 1H; Ar-H), 7.45 (d, J = $15.7 \mathrm{~Hz}, 1 \mathrm{H}$; CHCO), $7.33\left(\mathrm{t}, J=7.5 \mathrm{~Hz}, 1 \mathrm{H}\right.$; Ar-H), $6.96(\mathrm{~d}, J=8.7 \mathrm{~Hz}, 2 \mathrm{H} ; \mathrm{Ar}-\mathrm{H}), 3.87\left(\mathrm{~s}, 3 \mathrm{H} ; \mathrm{OCH}_{3}\right) .{ }^{13} \mathrm{C} \mathrm{NMR}\left(\mathrm{CDCl}_{3}, 100\right.$ $\mathrm{MHz}) \delta 179.6,161.9,155.6,153.8,144.4,130.5,128.0,127.3,123.8,123.1,118.7,114.4,112.7,112.3$, 55.3. IR $v 3137(\mathrm{w}), 3065(\mathrm{w}), 2844(\mathrm{w}), 1652$ (s), 1589 (s), 1571 (s), 1557 (s), 1510 (s), $1475(\mathrm{~m}), 1447$ (m), 1424 (s), 1361 (w), 1348 (m), 1295 (s), 1262 (s), 1248 (s), 1194 (m), 1177 (s), 1160 (s), 1139 (s), 1036 (s), $1024(\mathrm{~s}), 983$ (s), 934 (s), 910 (s), 880 (s), 855 (m), 819 (s), 784 (s), 735 (s). HRMS(ESI) calcd for $\mathrm{C}_{18} \mathrm{H}_{14} \mathrm{O}_{3}{ }^{+}(\mathrm{M}+\mathrm{H})$ 279.1016, found 279.0999.

\section{2-[2-(4-Methoxyphenyl)-1-cyclopropanecarbonyl]-benzofurane (8)}

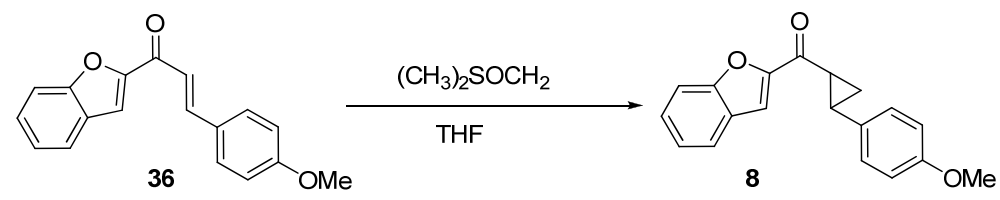

Following general procedure 3, a solution of sulphoxonium ylide $(5.9 \mathrm{~mL})$ was added to a solution of alkene 36 (800 mg, $2.90 \mathrm{mmol}, 1.00$ equiv) in THF $(30 \mathrm{~mL})$. The reaction was stirred at RT during $45 \mathrm{~min}$ then quenched. Purification by flash column chromatography (PET/AcOEt 4:1) gave 8 (384 mg, $1.31 \mathrm{mmol}$, $45 \%$ ) as white crystals. $R_{f} 0.80$ (PET/AcOEt 7:3, Anisaldehyde). Mp 115-117 ${ }^{\circ} \mathrm{C} .{ }^{1} \mathrm{H}$ NMR $\left(\mathrm{CDCl}_{3}, 400\right.$ MHz) $\delta 7.70(\mathrm{~d}, J=7.9 \mathrm{~Hz}, 1 \mathrm{H}$; Ar-H), $7.61-7.52$ (m, 2H; Ar-H), 7.47 (dd, J = 4.1, $11.4 \mathrm{~Hz}, 1 \mathrm{H}$; Ar-H), 7.31 (t, $J=7.5 \mathrm{~Hz}, 1 \mathrm{H} ; \mathrm{Ar}-\mathrm{H}), 7.13$ (d, $J=8.6 \mathrm{~Hz}, 2 \mathrm{H}$; Ar-H), 6.87 (d, $J=8.7 \mathrm{~Hz}, 2 \mathrm{H}$; Ar-H), 3.81 (s, 3H; $\left.\mathrm{OCH}_{3}\right), 3.00-2.84(\mathrm{~m}, 1 \mathrm{H}$; $\mathrm{CH}$ cyclopropane), 2.76 (ddd, $J=4.0,6.8,10.6 \mathrm{~Hz}, 1 \mathrm{H}$; $\mathrm{CH}$ cyclopropane), $1.99-1.87$ (m, $1 \mathrm{H} ; \mathrm{CH}_{2}$ cyclopropane), $1.62-1.53$ (m, $1 \mathrm{H} ; \mathrm{CH}_{2}$ cyclopropane). ${ }^{13} \mathrm{C} \mathrm{NMR}\left(\mathrm{CDCl}_{3}, 100\right.$ MHz) $\delta 189.1,158.4,155.6,153.0,132.0,128.0,127.4,127.1,123.8,123.2,114.0,112.4,112.4,55.2,29.8$, 
29.5, 19.2. IR v $3004(\mathrm{w}), 2935$ (w), 2835 (w), 1659 (s), 1613 (m), 1558 (s), 1516 (s), 1440 (m), 1397 (s), 1337 (m), 1294 (m), 1248 (s), 1180 (s), 1158 (s), 1140 (s), 1034 (s), 1001 (s), 932 (m), 888 (m), 830 (s), 801 (s), 749 (s), $688(\mathrm{~m})$. HRMS(ESI) calcd for $\mathrm{C}_{19} \mathrm{H}_{16} \mathrm{O}_{3}{ }^{+}(\mathrm{M}+\mathrm{H})$ 293.1172, found 293.1172.

\section{(2-Bromo-cyclohex-2-enyl)-trimethyl-silane (10)}

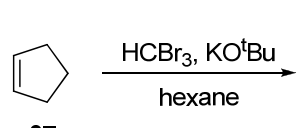

37

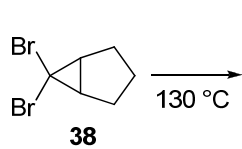

38

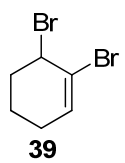

39
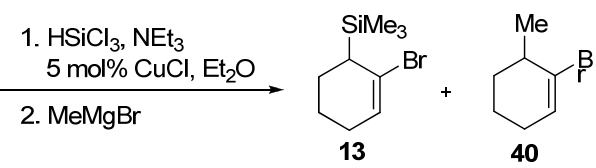

Following a reported procedure, ${ }^{9}$ bromoform $(12 \mathrm{~mL}, 0.14 \mathrm{mmol}, 1.0$ equiv) was added to a thick slurry of potassium tert-butoxide ( $17 \mathrm{~g}, 0.15 \mathrm{mmol}, 1.1$ equiv) and cyclopentene ( $13 \mathrm{~mL}, 0.15 \mathrm{mmol}, 1.1$ equiv) in dry hexane $(65 \mathrm{~mL})$ over $30 \mathrm{~min}$ at $0{ }^{\circ} \mathrm{C}$. The resulting yellow-brown suspension was warmed to $23{ }^{\circ} \mathrm{C}$ over $3 \mathrm{~h}$ and poured onto $300 \mathrm{~g}$ of ice. The mixture was extracted with PET $(3 \times 200 \mathrm{~mL})$. The combined organic layers were washed with water $(3 \times 200 \mathrm{~mL})$, dried over $\mathrm{MgSO}_{4}$ and the solvent was removed under reduced pressure. Distillation $\left(\mathrm{bp}=35-40{ }^{\circ} \mathrm{C}, \mathrm{p}=0.3 \mathrm{mbar}\right.$ gave cyclopropane 38 (23 g, $\left.96 \mathrm{mmol}, 69 \%\right)$ instead of the expected vinyl bromide $\mathbf{3 9}$ as a colorless oil. Quantitative conversion of $\mathbf{3 8}$ to the desired product $\mathbf{3 9}$ as a slightly brown oil was obtained by heating at $130{ }^{\circ} \mathrm{C}$ for $45 \mathrm{~min} .{ }^{1} \mathrm{H} \mathrm{NMR}\left(\mathrm{CDCl}_{3}, 400 \mathrm{MHz}\right) \delta 6.19(\mathrm{~m}$, 1H; alkene-H), $4.78(\mathrm{~m}, 1 \mathrm{H} ; \mathrm{CHBr}), 2.09-2.38\left(\mathrm{~m}, 6 \mathrm{H} ; \mathrm{CH}_{2}\right), 2.00\left(\mathrm{~m}, 1 \mathrm{H} ; \mathrm{CH}_{2}\right), 1.76\left(\mathrm{~m}, 1 \mathrm{H} ; \mathrm{CH}_{2}\right) .{ }^{13} \mathrm{C}$ NMR $\left(\mathrm{CDCl}_{3}, 100 \mathrm{MHz}\right) \delta$ 133.7, 122.2, 53.9, 33.6, 27.4, 16.3. IR $v 2953$ (w), 2935 (w), $2832(\mathrm{w}), 1634$ (w), 1437 (w), 1319 (w), 1191 (m), 996 (w), 943 (w), 907 (s), 889 (m), 847 (w), 730 (s), 638 (m). ${ }^{1} \mathrm{H}$ NMR, ${ }^{13} \mathrm{C}$ NMR corresponded to the literature values. ${ }^{10}$

Following a reported procedure, ${ }^{11}$ trichlorosilane $(1.1 \mathrm{~mL}, 11 \mathrm{mmol}, 1.1$ equiv) was added to a solution of allyl bromide 39 ( $2.4 \mathrm{~g}, 10 \mathrm{mmol}, 1.0$ equiv), triethylamine (dist. over $\mathrm{KOH}, 1.4 \mathrm{~mL}, 10 \mathrm{mmol}, 1.0$ equiv) and $\mathrm{CuCl}$ (49 $\mathrm{mg}, 0.50 \mathrm{mmol}, 0.050$ equiv) in $\mathrm{Et}_{2} \mathrm{O}(5 \mathrm{~mL})$ at $15{ }^{\circ} \mathrm{C}$. The resulting thick suspension was stirred at $23{ }^{\circ} \mathrm{C}$ for $4 \mathrm{~h}$ and filtered under nitrogen. The filter cake was washed with $\mathrm{Et}_{2} \mathrm{O}(2 \times 5 \mathrm{~mL})$, the combined filtrates were cooled to $0{ }^{\circ} \mathrm{C}$ and $\mathrm{MeMgBr}\left(3 \mathrm{M}\right.$ in $\mathrm{Et}_{2} \mathrm{O}, 13.3 \mathrm{~mL}, 40.0 \mathrm{mmol}, 4.00$ equiv) was added dropwise. The resulting red-green suspension was stirred for $1 \mathrm{~h}$ at $23{ }^{\circ} \mathrm{C}$ and poured onto sat. $\mathrm{NH}_{4} \mathrm{Cl}$ solution $(50 \mathrm{~mL})$ at $0{ }^{\circ} \mathrm{C}$. The mixture was extracted with $\mathrm{Et}_{2} \mathrm{O}(3 \times 50 \mathrm{~mL})$. The combined organic layers were washed with brine $(50 \mathrm{~mL})$, dried over $\mathrm{MgSO}_{4}$ and the solvent was removed under reduced pressure. The crude product was purified by flash column chromatography (PET) to yield allylsilane $\mathbf{1 0}(0.85 \mathrm{~g}, 3.7$ mmol, 37\%) contaminated by the corresponding methyl addition product $\mathbf{4 0}(0.45 \mathrm{~g}, 2.6 \mathrm{mmol}, 26 \%)$ as colorless oils. Allyl silane 10 of $93 \%$ purity $(0.71 \mathrm{~g}, 3.1 \mathrm{mmol}, 31 \%)$ was obtained after stirring $1 \mathrm{~h}$ in $\mathrm{HV}$ (0.3 mbar). ${ }^{1} \mathrm{H}$ NMR $\left(\mathrm{CDCl}_{3}, 400 \mathrm{MHz}\right) \delta 5.92\left(\mathrm{td}, J=3.8,1.6 \mathrm{~Hz}, 1 \mathrm{H}\right.$; alkene-H), 1.95-2.20 (m, $\left.3 \mathrm{H} ; \mathrm{CH}_{2}\right)$, 1.82-1.94 (m, 1H; $\left.\mathrm{CH}_{2}\right), 1.67-1.80\left(\mathrm{~m}, 1 \mathrm{H} ; \mathrm{CH}_{2}\right), 1.47-1.64\left(\mathrm{~m}, 2 \mathrm{H} ; \mathrm{CH}_{2}\right), 0.14\left(\mathrm{~s}, 9 \mathrm{H}, \mathrm{SiCH}_{3}\right) .{ }^{13} \mathrm{C} \mathrm{NMR}$ $\left(\mathrm{CDCl}_{3}, 100 \mathrm{MHz}\right) \delta 126.1,125.4,35.3,27.4,27.3,20.6,-1.0 . \mathrm{IR} v 2934(\mathrm{~m}), 2858(\mathrm{w}), 1637(\mathrm{w}), 1451(\mathrm{w})$, 1249 (s), 1055 (w), 1031 (w), 986 (m), 940 (w), 919 (w), 836 (s), 798 (m), 733 (m), 692 (w), 615 (w). ${ }^{1} \mathrm{H}$ NMR, ${ }^{13} \mathrm{C}$ NMR corresponded to the literature values. ${ }^{12}$

\section{2-[2-(2,4-Dimethoxyphenyl)-1-cyclopropanecarbonyl]-3-trimethylsilyl-cyclohex-2-ene (11)}

\footnotetext{
${ }^{9}$ Stevens, C. L.; Valicent.Ja J. Am. Chem. Soc. 1965, 87, 838.

${ }^{10}$ Banwell, M. G.; Cowden, C. J. Aust. J. Chem. 1994, 47, 2235.

${ }^{11}$ Heerding, D. A.; Hong, C. Y.; Kado, N.; Look, G. C.; Overman, L. E. J. Org. Chem.1993, 58, 6947.

${ }^{12}$ Denmark, S. E.; Klix, R. C. Tetrahedron 1988, 44, 4043.
} 


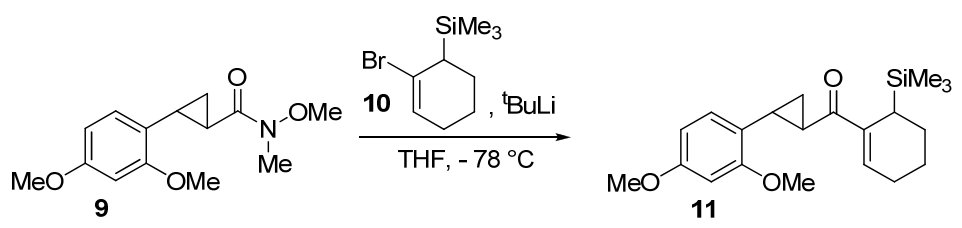

Amide 9 (363 mg, $1.37 \mathrm{mmol}, 1.00$ equiv) was added to vinyl bromide 10 (320 mg, $1.37 \mathrm{mmol}, 1.00$ equiv), following general procedure 4. The deprotonation time was $30 \mathrm{~min}$ at $-78^{\circ} \mathrm{C}$ and the reaction was quenched after $1 \mathrm{~h}$ to give $\mathbf{1 1}(250 \mathrm{mg}, 0.70 \mathrm{mmol}, 51 \%)$ as colorless oil without further purification. $R_{f} 0.25$ (PET/AcOEt 15:1, Anisaldehyde). ${ }^{1} \mathrm{H}$ NMR $\left(\mathrm{CDCl}_{3}, 400 \mathrm{MHz}\right) \delta 6.89$ (m, 1H; Ar-H), 6.82 (m, 1H; Ar-H), $6.42(\mathrm{~m}, 1 \mathrm{H} ; \mathrm{Ar}-\mathrm{H}), 6.39\left(\mathrm{~m}, 1 \mathrm{H}\right.$; alkene-H), $3.79\left(\mathrm{~s}, 3 \mathrm{H} ; \mathrm{OCH}_{3}\right), 3.75\left(\mathrm{~s}, 3 \mathrm{H} ; \mathrm{OCH}_{3}\right), 2.59(\mathrm{t}, J=9.8 \mathrm{~Hz}$, $1 \mathrm{H}$; $\mathrm{CH}$ cyclopropane), $2.45-2.32\left(\mathrm{~m}, 2 \mathrm{H}\right.$; $\mathrm{CH}$ cyclopropane, $\mathrm{CH}_{2}$ cyclohexene), $2.22\left(\mathrm{~m}, 2 \mathrm{H} ; \mathrm{CH}_{2}\right.$ cyclohexene), 1.87 (m, 1H; $\mathrm{CH}_{2}$ cyclopropane), 1.64- 1.49 (m, 5H, $\mathrm{CH}_{2}$ cyclohexene), 1.37 (td, $J=3.9,7.4$ $\mathrm{Hz}, 0.7 \mathrm{H} ; \mathrm{CH}_{2}$ cyclopropane, Diasteroisomer A), $1.33-1.24\left(\mathrm{~m}, 0.3 \mathrm{H} ; \mathrm{CH}_{2}\right.$ cyclopropane, Diasteroisomer B), $0.94-0.76$ (m, 1H; CHSi), 0.01 (s, 7H, $\mathrm{SiCH}_{3}$ Diasteroisomer A), -0.02 (s, 2H; $\mathrm{SiCH}_{3}$ Diasteroisomer B). From $0 \mathrm{ppm}$ to $2.6 \mathrm{ppm}$ we found several overlaps due to presence of 2 diastereoisomers. ${ }^{13} \mathrm{C}$ NMR $\left(\mathrm{CDCl}_{3}, 75 \mathrm{MHz}\right) \delta 200.0,159.5,143.6,135.8,135.3,126.8,126.7,121.6,103.5,103.8,98.4,98.3,55.4$, $55.1,26.8,26.5,25.7,25.7,24.1,24.0,23.9,23.8,23.2,23.2,20.6,, 16.8,16.1,-1.1$. The excess of signals is due to presence of 2 diastereoisomers. IR $v 3003(\mathrm{w}), 2937(\mathrm{w}), 2836(\mathrm{w}), 1648(\mathrm{~m}), 1614(\mathrm{~m}), 1585(\mathrm{w})$, 1509 (m), 1456 (w), 1436 (w), 1402 (m), 1291 (m), 1248 (m), 1208 (s), 1186 (m), 1159 (m), 1123 (w), 1034 (m), 990 (w), 955 (m), $920(\mathrm{w}), 835$ (s), 796 (w), 752 (w), 731 (w). HRMS(ESI) calcd for $\mathrm{C}_{21} \mathrm{H}_{30} \mathrm{O}_{3} \mathrm{Si}^{+}$ $(\mathrm{M}+\mathrm{H})$ 359.2037, found 359.2044.

\section{1-Bromocyclohexene (42)}

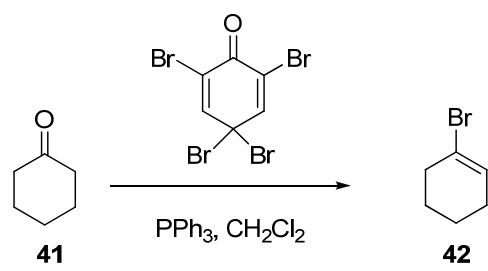

Following a reported procedure, ${ }^{13}$ a mixture of cyclohexenone (distilled, $0.50 \mathrm{~g}, 5.1 \mathrm{mmol}, 1.0$ equiv), triphenylphosphine (3.2 g, $12 \mathrm{mmol}, 2.4$ equiv) and 2,4,4,6-tetrabromo-2,5-cyclohexadienone (4.9 g, $12 \mathrm{mmol}$, 2.4 equiv) was refluxed in $\mathrm{CH}_{2} \mathrm{Cl}_{2}(15 \mathrm{~mL})$ for $18 \mathrm{~h}$. The crude mixture was filtered over $\mathrm{SiO}_{2}$, washed with $\mathrm{CH}_{2} \mathrm{Cl}_{2}$ and the solvent was removed under reduced pressure. The crude product was purified by flash column chromatography (PET/Et ${ }_{2} \mathrm{O}$ 100:1) to yield 1-bromocyclohexene (42) (354 mg, 90\% pure by ${ }^{1} \mathrm{HNMR}, 1.98$ mmol, 39\%). $\left.R_{f} 0.80 \mathrm{PET}_{/} \mathrm{Et}_{2} \mathrm{O} 100: 1, \mathrm{KMNO}_{4}\right) .{ }^{1} \mathrm{H} \mathrm{NMR}\left(\mathrm{CDCl}_{3}, 400 \mathrm{MHz}\right) \delta 6.03(\mathrm{~m}, 1 \mathrm{H}$, alkene $\mathrm{H}), 2.36-2.48(\mathrm{~m}, 2$ $\left.\mathrm{H}, \mathrm{CH}_{2}\right), 2.02-2.12\left(\mathrm{~m}, 2 \mathrm{H}, \mathrm{CH}_{2}\right), 1.68-1.79\left(\mathrm{~m}, 2 \mathrm{H}, \mathrm{CH}_{2}\right), 1.54-1.67\left(\mathrm{~m}, 2 \mathrm{H}, \mathrm{CH}_{2}\right)^{13} \mathrm{CNMR}\left(\mathrm{CDCl}_{3}, 100 \mathrm{MHz}\right) \delta 128.9$, 122.3, 35.2, 27.4, 24.5, 21.1. ${ }^{1} \mathrm{H} \mathrm{NMR},{ }^{13} \mathrm{C}$ NMR corresponded to the literature values. ${ }^{13}$

\section{Cyclohex-1-enyl-[2-(4-methoxy-phenyl)-cyclopropyl]-methanone (15)}

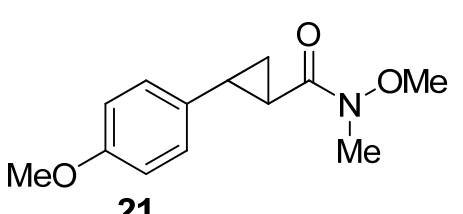

21

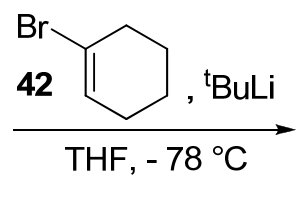

$\mathrm{THF},-78^{\circ} \mathrm{C}$

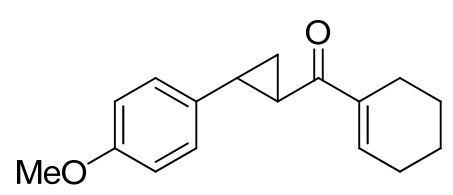

15

$\left({ }^{13}\right)$ Matveeva, E. D.; Feshin, D. B.; Zefirov, N. S. Russ. J. Org. Chem. 2001, 37, 52. 
Amide 21 (445 mg, $1.68 \mathrm{mmol}, 1.00$ equiv) was added to vinyl bromide 42 (300 mg, 90\% pure, 1.68 $\mathrm{mmol}, 1.00$ equiv), following general procedure 4 . The deprotonation time was $30 \mathrm{~min}$ at $-78^{\circ} \mathrm{C}$ and the reaction was quenched after $2 \mathrm{~h}$ to give $15(275 \mathrm{mg}, 1.07 \mathrm{mmol}, 64 \%)$ as a colorless solid after purification on column chromatography (PET/AcOEt 10:1). ( $R_{f} 0.25$ (PET/AcOEt 15:1, Anisaldehyde). ${ }^{1} \mathrm{H}$ NMR $\left(\mathrm{CDCl}_{3}, 400 \mathrm{MHz}\right) \delta 7.05(\mathrm{~d}, J=8.8 \mathrm{~Hz}, 2 \mathrm{H}, \mathrm{Ar} \mathrm{H}), 6.99$ (br m, $1 \mathrm{H}$, alkene H), $6.83(\mathrm{~d}, J=8.8 \mathrm{~Hz}, 2 \mathrm{H}, \mathrm{Ar}$ $\mathrm{H}), 3.79\left(\mathrm{~s}, 3 \mathrm{H}, \mathrm{OCH}_{3}\right), 2.51(\mathrm{~m}, 1 \mathrm{H}$, cyclopropane $\mathrm{H}), 2.45(\mathrm{~m}, 1 \mathrm{H}$, cyclopropane $\mathrm{H}), 2.21-2.33(\mathrm{~m}, 4 \mathrm{H}$, cyclohexene $\left.\mathrm{CH}_{2}\right), 1.57-1.73\left(\mathrm{~m}, 5 \mathrm{H}\right.$, Cyclohexene and cyclopropane $\left.\mathrm{CH}_{2}\right), 1.30$ (ddd, $J=8.1,6.5,4.0 \mathrm{~Hz}$, $1 \mathrm{H}$, cyclopropane $\left.\mathrm{CH}_{2}\right) .{ }^{13} \mathrm{C} \mathrm{NMR}\left(\mathrm{CDCl}_{3}, 75 \mathrm{MHz}\right) \delta 199.1,158.2,139.8,139.7,132.9,127.2,113.8,55.3$, 28.3, 27.5, 26.1, 23.5, 21.9, 21.6, 18.2. IR v $3004(\mathrm{w}), 2935$ (m), 2934 (m), $2863(\mathrm{w}), 2862(\mathrm{w}), 2836(\mathrm{w})$, 2835 (w), 1650 (s), 1649 (s), 1517 (s), 1405 (m), 1250 (s), 1205 (m), 1037 (w), 1031 (w), 917 (w), 837 (w), 741 (w), 736 (w). HRMS(ESI) calcd for $\mathrm{C}_{17} \mathrm{H}_{21} \mathrm{O}_{2}{ }^{+}(\mathrm{M}+\mathrm{H})$ 257.1536, found 257.1543.

\section{Cyclization}

\section{5-(4-Methoxyphenyl)-3,4,6,7-tetrahydro-2H-chromen-8(5H)-one (3a)}

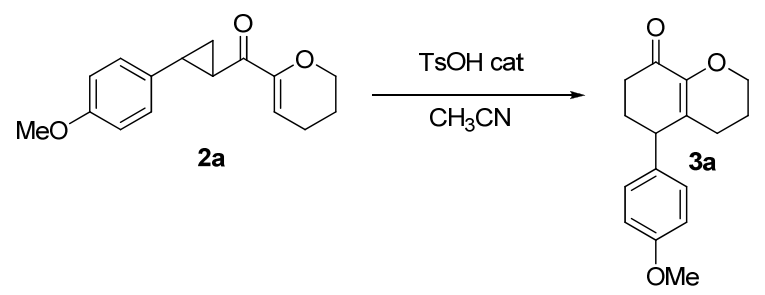

The reaction was carried out following general procedure 5, starting from cyclopropane 2a (103 mg, $0.400 \mathrm{mmol}, 1.00$ equiv) and tosic acid (15 $\mathrm{mg}, 0.080 \mathrm{mmol}, 0.20$ equiv). The reaction was quenched after $18 \mathrm{~h}$. Purification by flash chromatography (PET/AcOEt, 4:1) afforded 3a (72 mg, $0.28 \mathrm{mmol}, 70 \%$ ) as yellow oil. $R_{f} 0.35$ (PET/AcOEt 1:1, Anisaldehyde). ${ }^{1} \mathrm{H} \mathrm{NMR}\left(\mathrm{CDCl}_{3}, 400 \mathrm{MHz}\right) \delta 7.12(\mathrm{~d}, J=8.6 \mathrm{~Hz}, 2 \mathrm{H}$; Ar-H), 6.88 (d, $J=8.6 \mathrm{~Hz}, 2 \mathrm{H}$; Ar-H), $4.20-3.99\left(\mathrm{~m}, 2 \mathrm{H} ; \mathrm{CH}_{2} \mathrm{O}\right), 3.81\left(\mathrm{~s}, 3 \mathrm{H}, \mathrm{OCH}_{3}\right), 3.58(\mathrm{t}, J=5.2 \mathrm{~Hz}$, $1 \mathrm{H} ; \mathrm{CH}-\mathrm{Ar}), 2.59-2.47$ (m, 1H; $\left.\mathrm{CH}_{2}\right), 2.45-2.27\left(\mathrm{~m}, 2 \mathrm{H} ; \mathrm{CH}_{2}\right), 2.03-1.90\left(\mathrm{~m}, 3 \mathrm{H} ; \mathrm{CH}_{2}\right), 1.85(\mathrm{~d}, J=6.0$ $\mathrm{Hz}, 2 \mathrm{H} ; \mathrm{CH}_{2}$ ). ${ }^{1} \mathrm{H}$ NMR (benzene-d $\left.{ }^{6}, 400 \mathrm{MHz}\right) \delta$ 6.83-6.77 (m, 2H, Ar-H), 6.76-6.70 (m, 2H; Ar-H), 3.75$3.57\left(\mathrm{~m}, 2 \mathrm{H} ; \mathrm{CH}_{2} \mathrm{O}\right), 3.33\left(\mathrm{~s}, 3 \mathrm{H}, \mathrm{OCH}_{3}\right), 3.02$ (dd, $J=6.00,5.29 \mathrm{~Hz}, 1 \mathrm{H}, \mathrm{CH}-\mathrm{Ar}$ ), 2.37 (ddd, $J=16.61$, 9.59, $4.50 \mathrm{~Hz}, 1 \mathrm{H}, \mathrm{CH}_{2}$-ketone), 2.14 (ddd, $J=16.58,8.04,4.49 \mathrm{~Hz}, 1 \mathrm{H}, \mathrm{CH}_{2}$-ketone), 1.85 (tdd, $J=13.29$, 9.57, $\left.4.65 \mathrm{~Hz}, 1 \mathrm{H}, \mathrm{CH}_{2}\right), 1.62-1.49\left(\mathrm{~m}, 2 \mathrm{H}, \mathrm{CH}_{2}\right), 1.45-1.35\left(\mathrm{~m}, 1 \mathrm{H}, \mathrm{CH}_{2}\right), 1.32-1.20\left(\mathrm{~m}, 2 \mathrm{H}, \mathrm{CH}_{2}\right) .{ }^{13} \mathrm{C}$ $\mathrm{NMR}\left(\mathrm{CDCl}_{3}, 100 \mathrm{MHz}\right) \delta 193.2,158.5,146.8,133.3,132.1,128.9,114.1,65.8,55.2,45.0,34.9,30.9$, 25.1, 21.8. IR v $2934(\mathrm{~m}), 2870(\mathrm{w}), 1683$ (s), 1612 (w), 1511 (s), 1463 (w), 1385 (w), 1293 (w), 1247 (s), $1180(\mathrm{~m}), 1154(\mathrm{~m}), 1085(\mathrm{w}), 1035(\mathrm{~m}), 986(\mathrm{w}), 926(\mathrm{w}), 833(\mathrm{~m})$. HRMS(ESI) calcd for $\mathrm{C}_{16} \mathrm{H}_{18} \mathrm{O}_{3}{ }^{+}$ $(\mathrm{M}+\mathrm{H})$ 259.1329, found 259.1323.

\section{Deuterium Labeling Experiment}

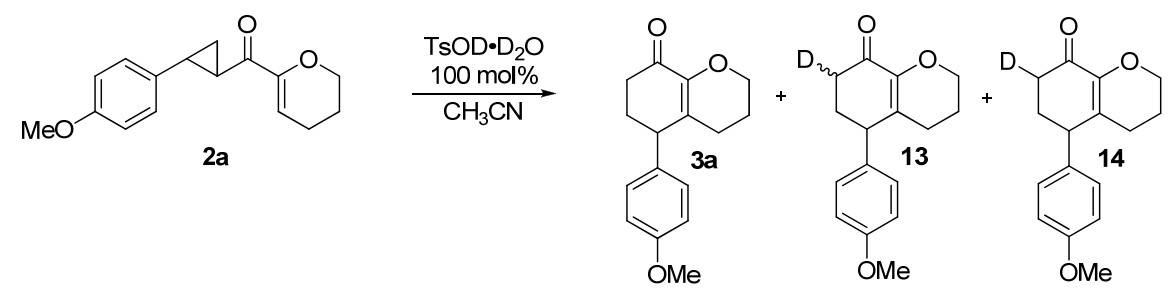


The reaction was carried out following general procedure 5, starting from cyclopropane 2a (33 mg, 0.13 mmol, 1.0 equiv) and deuterated tosic acid monohydrate (23 mg, $0.13 \mathrm{mmol}, 1.0$ equiv). The reaction was quenched after $1 \mathrm{~h}$. Purification by flash chromatography (PET/AcOEt, 4:1) afforded a mixture of 3a, 13 and 14 (20 mg, $0.078 \mathrm{mmol}, 61 \%$ ) as yellow oil. $R_{f} 0.35$ (PET/AcOEt 1:1, Anisaldehyde). ). ${ }^{1} \mathrm{H}$ NMR (benzene$\left.\mathrm{d}^{6}, 400 \mathrm{MHz}\right) \delta$ 6.83-6.77 (m, 2H, Ar-H), 6.76-6.70 (m, 2H; Ar-H), 3.75-3.57 (m, 2H; $\left.\mathrm{CH}_{2} \mathrm{O}\right), 3.33(\mathrm{~s}, 3 \mathrm{H}$, $\mathrm{OCH}_{3}$ ), 3.02 (m, 1H, CH-Ar), 2.37 (ddd, $J=16.61,9.59,4.50 \mathrm{~Hz}, 0.6 \mathrm{H}, \mathrm{CH}_{2}$-ketone), 2.14 (ddd, $J=16.58$, 8.04, $4.49 \mathrm{~Hz}, 0.6 \mathrm{H}, \mathrm{CH}_{2}$-ketone), $1.85\left(\mathrm{~m}, 1 \mathrm{H}, \mathrm{CH}_{2}\right), 1.62-1.49\left(\mathrm{~m}, 2 \mathrm{H}, \mathrm{CH}_{2}\right), 1.45-1.35\left(\mathrm{~m}, 1 \mathrm{H}, \mathrm{CH}_{2}\right)$, 1.32-1.20 (m, 2H, $\left.\mathrm{CH}_{2}\right) .{ }^{13} \mathrm{C} \mathrm{NMR}\left(\mathrm{CDCl}_{3}, 100 \mathrm{MHz}\right) \delta 193.33,193.29,193.24,158.6,146.8,133.36$, 133.331, 133.30, 133.26, 132.16, 132.13, 129.0, 114.1, 65.9, 55.3, 45.06, 45.03, 45.00, 44.97, 34.9, 34.5 (t, J $=19.5 \mathrm{~Hz}), 30.91,30.85,30.81,30.74,29.7,25.1,21.8$. HRMS(ESI) calcd for $\mathrm{C}_{16} \mathrm{H}_{18} \mathrm{NaO}_{3}{ }^{+}(\mathrm{M}+\mathrm{Na})$ 281.1148, found 281.1141 (87\%), calcd for $\mathrm{C}_{16} \mathrm{H}_{17} \mathrm{DNaO}_{3}{ }^{+}(\mathrm{MD}+\mathrm{Na}) 282.1211$, found 282.1205 (100\%), calcd for $\mathrm{C}_{16} \mathrm{H}_{16} \mathrm{D}_{2} \mathrm{NaO}_{3}{ }^{+}\left(\mathrm{MD}_{2}+\mathrm{Na}\right) 283.1274$, found $283.1278(70 \%)$.

\section{5-(3,4-Dimethoxyphenyl)-3,4,6,7-tetrahydro-2H-chromen-8(5H)-one (3c)}

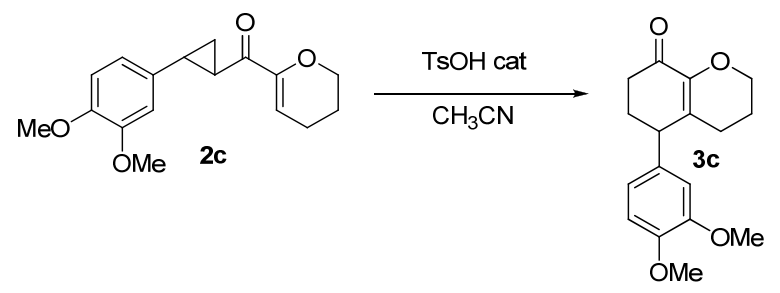

The reaction was performed following general procedure 5, starting from cyclopropane 2c (115 mg, $0.400 \mathrm{mmol}, 1.00$ equiv) and tosic acid (15 mg, $0.080 \mathrm{mmol}, 0.20$ equiv). The reaction was quenched after 5 $\mathrm{h}$ to give 3c (115 mg, $0.400 \mathrm{mmol}, 100 \%$ ) as yellow oil without further purification. $R_{f} 0.25$ (PET/AcOEt 1:1, Anisaldehyde). ${ }^{1} \mathrm{H}$ NMR $\left(\mathrm{CDCl}_{3}, 400 \mathrm{MHz}\right) \delta 6.82(\mathrm{~d}, J=8.2 \mathrm{~Hz}, 1 \mathrm{H} ; \mathrm{Ar}-\mathrm{H}), 6.76-6.68(\mathrm{~m}, 2 \mathrm{H}$; Ar$\mathrm{H}), 4.17$ - $4.02\left(\mathrm{~m}, 2 \mathrm{H} ; \mathrm{CH}_{2} \mathrm{O}\right), 3.87\left(\mathrm{~s}, 3 \mathrm{H} ; \mathrm{OCH}_{3}\right), 3.86\left(\mathrm{~s}, 3 \mathrm{H} ; \mathrm{OCH}_{3}\right), 3.57(\mathrm{t}, J=5.3 \mathrm{~Hz}, 1 \mathrm{H} ; \mathrm{CH} \mathrm{Ar})$ $2.54\left(\mathrm{ddd}, J=4.3,9.5,16.2 \mathrm{~Hz}, 1 \mathrm{H} ; \mathrm{CH}_{2}\right), 2.46-2.28\left(\mathrm{~m}, 2 \mathrm{H} ; \mathrm{CH}_{2}\right), 2.03-1.92\left(\mathrm{~m}, 3 \mathrm{H} ; \mathrm{CH}_{2}\right), 1.91-1.78$ $\left(\mathrm{m}, 2 \mathrm{H} ; \mathrm{CH}_{2}\right) .{ }^{13} \mathrm{C} \mathrm{NMR}\left(\mathrm{CDCl}_{3}, 100 \mathrm{MHz}\right) \delta 193.1,149.1,146.7,144.9,133.7,131.9,119.8,111.1,65.8$, 55.8, 55.8, 45.3, 34.9, 30.7, 25.0, 21.7. IR $v 2936(\mathrm{w}), 2871(\mathrm{w}), 2835(\mathrm{w}), 1731(\mathrm{w}), 1678(\mathrm{~s}), 1629(\mathrm{w})$, $1592(\mathrm{w}), 1515$ (s), 1464 (m), $1450(\mathrm{w}), 1418(\mathrm{w}), 1385(\mathrm{w}), 1279(\mathrm{w}), 1265$ (m), 1248 (m), 1233 (m), 1182 (m), 1139 (s), 1085 (w), $1026(\mathrm{~s}), 987(\mathrm{~m}), 913(\mathrm{~m}), 851(\mathrm{w}), 812$ (w), 728 (s). HRMS(ESI) calcd for $\mathrm{C}_{17} \mathrm{H}_{20} \mathrm{O}_{4}{ }^{+}(\mathrm{M}+\mathrm{H})$ 289.1434, found 289.1420.

\section{5-(2,4-Dimethoxyphenyl)-3,4,6,7-tetrahydro-2H-chromen-8(5H)-one (3d)}

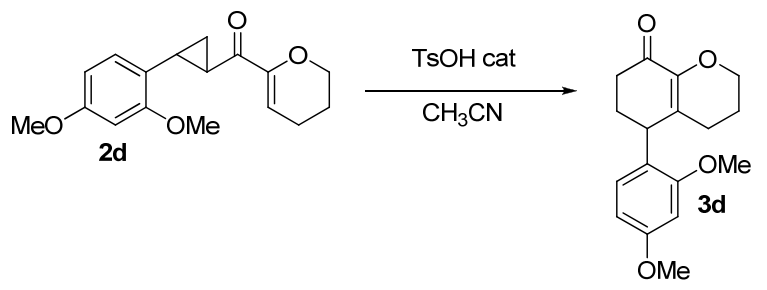

The cyclization was achieved following general procedure 5, starting from cyclopropane $2 \mathbf{d}$ (115 mg, $0.400 \mathrm{mmol}, 1.00$ equiv) and tosic acid (15 $\mathrm{mg}, 0.080 \mathrm{mmol}, 0.20$ equiv). The reaction was quenched after $10 \mathrm{~min}$ to give $3 \mathbf{d}(115 \mathrm{mg}, 0.400 \mathrm{mmol}, 100 \%)$ as yellow oil without further purification. $R_{f} 0.40$ (PET/AcOEt 1:1, Anisaldehyde). ${ }^{1} \mathrm{H} \mathrm{NMR}\left(\mathrm{CDCl}_{3}, 400 \mathrm{MHz}\right) \delta 6.82(\mathrm{~d}, J=8.2 \mathrm{~Hz}, 1 \mathrm{H} ; \mathrm{Ar}-\mathrm{H}), 6.77-6.68$ (m, 2H; Ar-H), 4.19 - $4.01\left(\mathrm{~m}, 2 \mathrm{H} ; \mathrm{CH}_{2} \mathrm{O}\right), 3.87\left(\mathrm{~s}, 3 \mathrm{H} ; \mathrm{OCH}_{3}\right), 3.86\left(\mathrm{~s}, 3 \mathrm{H} ; \mathrm{OCH}_{3}\right), 3.57$ (t, J = 5.3 Hz, 1H; 
CH Ar), 2.54 (ddd, $\left.J=4.3,9.5,16.2 \mathrm{~Hz}, 1 \mathrm{H} ; \mathrm{CH}_{2}\right), 2.46-2.28\left(\mathrm{~m}, 2 \mathrm{H} ; \mathrm{CH}_{2}\right), 2.04-1.92\left(\mathrm{~m}, 3 \mathrm{H} ; \mathrm{CH}_{2}\right)$, $1.91-1.77\left(\mathrm{~m}, 2 \mathrm{H} ; \mathrm{CH}_{2}\right) .{ }^{13} \mathrm{C} \mathrm{NMR}\left(\mathrm{CDCl}_{3}, 100 \mathrm{MHz}\right) \delta 193.8,159.8,158.3,147.0,132.8,128.4,120.9$, 103.7, 98.8, 65.8, 55.3, 55.2, 38.3, 34.9, 28.2, 24.9, 21.9. IR v 2961 (w), $2937(\mathrm{w}), 2837$ (w), $1674(\mathrm{~m}), 1612$ (w), $1587(\mathrm{w}), 1505(\mathrm{w}), 1465(\mathrm{w}), 1438(\mathrm{w}), 1419(\mathrm{w}), 1388(\mathrm{w}), 1293(\mathrm{w}), 1259(\mathrm{w}), 1208(\mathrm{~m}), 1158(\mathrm{~m})$, $1115(\mathrm{w}), 1087(\mathrm{w}), 1036(\mathrm{w}), 986(\mathrm{w}), 907$ (s), $838(\mathrm{w}), 827(\mathrm{w}), 726(\mathrm{~s}), 648(\mathrm{~m})$. HRMS(ESI) calcd for $\mathrm{C}_{17} \mathrm{H}_{20} \mathrm{O}_{4}{ }^{+}(\mathrm{M}+\mathrm{H})$ 289.1434, found 289.1444.

\section{5-(Furan-2-yl)-3,4,6,7-tetrahydro-2H-chromen-8(5H)-one (3e)}

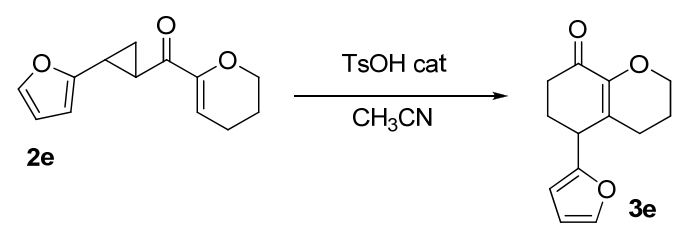

The cyclization was achieved following general procedure 6, starting from cyclopropane $2 \mathbf{e}(87.3 \mathrm{mg}$, $0.400 \mathrm{mmol}, 1.00$ equiv) and tosic acid ( $15 \mathrm{mg}, 0.080 \mathrm{mmol}, 0.20$ equiv). The reaction was quenched after 2 h. Purification by flash chromatography (PET/AcOEt, 2:8) afforded3 (44 mg, $0.20 \mathrm{mmol}, 50 \%$ ) as yellow oil. $R_{f} 0.10$ (PET/AcOEt 8:2, Anisaldehyde). ${ }^{1} \mathrm{H}$ NMR $\left(\mathrm{CDCl}_{3}, 400 \mathrm{MHz}\right) \delta 7.35$ (s, 1H; Ar-H), $6.36-6.26$ $(\mathrm{m}, 1 \mathrm{H} ; \mathrm{Ar}-\mathrm{H}), 6.07$ (d, J = 3.1 Hz, 1H; Ar-H), $4.13-4.01\left(\mathrm{~m}, 2 \mathrm{H} ; \mathrm{CH}_{2} \mathrm{O}\right), 3.68$ (t, J = 4.9 Hz, 1H; CH Ar), $2.68-2.51\left(\mathrm{~m}, 1 \mathrm{H} ; \mathrm{CH}_{2}\right), 2.47-2.35\left(\mathrm{~m}, 1 \mathrm{H} ; \mathrm{CH}_{2}\right), 2.32-2.13\left(\mathrm{~m}, 2 \mathrm{H} ; \mathrm{CH}_{2}\right), 2.10(\mathrm{t}, J=6.4 \mathrm{~Hz}, 2 \mathrm{H}$; $\left.\mathrm{CH}_{2}\right), 1.86\left(\mathrm{dq}, J=3.6,6.6 \mathrm{~Hz}, 2 \mathrm{H} ; \mathrm{CH}_{2}\right) .{ }^{13} \mathrm{C} \mathrm{NMR}\left(\mathrm{CDCl}_{3}, 100 \mathrm{MHz}\right) \delta 192.8,154.4,146.5,142.0,130.0$, 110.2, 106.7, 65.9, 39.2, 35.1, 29.6, 24.8, 21.8. IR $v 2933(\mathrm{w}), 2874(\mathrm{w}), 1685(\mathrm{~m}), 1635(\mathrm{w}), 1505(\mathrm{w})$, $1385(\mathrm{w}), 1289(\mathrm{w}), 1146(\mathrm{w}), 1087(\mathrm{w}), 1043(\mathrm{w}), 1009(\mathrm{w}), 985(\mathrm{w}), 911(\mathrm{~m}), 854(\mathrm{w}), 810(\mathrm{w}), 730(\mathrm{~s})$. HRMS(ESI) calcd for $\mathrm{C}_{13} \mathrm{H}_{14} \mathrm{O}_{3}{ }^{+}(\mathrm{M}+\mathrm{H}) 219.1016$, found 219.1007 .

\section{5-(4-Methoxyphenyl)-7-methyl-3,4,6,7-tetrahydro-2H-chromen-8(5H)-one (3f)}

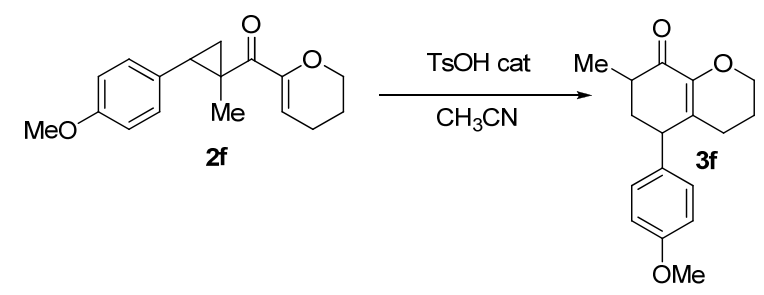

The reaction was carried out following general procedure 5, starting from cyclopropane $2 \mathrm{f}$ (64.0 $\mathrm{mg}$, $0.235 \mathrm{mmol}, 1.00$ equiv) and tosic acid $(9.0 \mathrm{mg}, 0.047 \mathrm{mmol}, 0.20$ equiv). The reaction was quenched after $1 \mathrm{~h}$. Purification by flash chromatography (PET/AcOEt, 8/2) afforded 3f $(64.0 \mathrm{mg}, 0.235 \mathrm{mmol}, 100 \%$, 5:1 mixture of diastereoisomers) as yellow oil. $R_{f} 0.15$ (PET/AcOEt 8:2, Anisaldehyde). ${ }^{1} \mathrm{H}$ NMR $\left(\mathrm{CDCl}_{3}, 400\right.$ $\mathrm{MHz}$ ) (Major diastereoisomer) $\delta 7.10(\mathrm{~d}, J=8.4 \mathrm{~Hz}, 2 \mathrm{H}$; Ar-H), $6.86(\mathrm{~d}, J=8.5 \mathrm{~Hz}, 2 \mathrm{H}$; Ar-H), 4.26 (d, $J$ $\left.=8.8 \mathrm{~Hz}, 1 \mathrm{H} ; \mathrm{CH}_{2} \mathrm{O}\right), 3.95-3.81\left(\mathrm{~m}, 1 \mathrm{H} ; \mathrm{CH}_{2} \mathrm{O}\right), 3.79\left(\mathrm{~s}, 3 \mathrm{H} ; \mathrm{OCH}_{3}\right), 3.55(\mathrm{~d}, J=7.3 \mathrm{~Hz}, 1 \mathrm{H} ; \mathrm{CH} \mathrm{Ar})$, $2.64-2.40\left(\mathrm{~m}, 1 \mathrm{H} ; \mathrm{CH}_{2}\right), 2.14\left(\mathrm{~m}, 1 \mathrm{H} ; \mathrm{CH}_{2}\right), 1.79\left(\mathrm{~m}, 4 \mathrm{H} ; \mathrm{CH}_{2}\right), 1.15\left(\mathrm{~m}, 3 \mathrm{H} ; \mathrm{CH}_{3}\right) .{ }^{13} \mathrm{C} \mathrm{NMR}\left(\mathrm{CDCl}_{3}, 100\right.$ MHz) $\delta 195.4,158.4,146.3135 .1,131.6,129.0,128.5$ 114.0, 113.6, 65.7, 55.2, 46.0, 41.3, 40.3, 25.0, 21.7, 14.9. IR v $2961(\mathrm{w}), 2932(\mathrm{w}), 2870(\mathrm{w}), 2836(\mathrm{w}), 1729(\mathrm{w}), 1682(\mathrm{~s}), 1623(\mathrm{~m}), 1611(\mathrm{w}), 1584(\mathrm{w}), 1512$ (s), $1458(\mathrm{w}), 1444(\mathrm{w}), 1385(\mathrm{w}), 1272(\mathrm{~m}), 1250(\mathrm{~s}), 1178(\mathrm{~m}), 1149(\mathrm{~s}), 1093(\mathrm{w}), 1079(\mathrm{w}), 1035(\mathrm{~m})$, 990 (m), 919 (w), 855 (w), 833 (m), 732 (m). HRMS(ESI) calcd for $\mathrm{C}_{17} \mathrm{H}_{20} \mathrm{O}_{3}{ }^{+}(\mathrm{M}+\mathrm{H})$ 273.1485, found 273.1490 .

\section{4-(4-Methoxyphenyl)-2,3,5,6-tetrahydrobenzofuran-7(4H)-one (5a)}




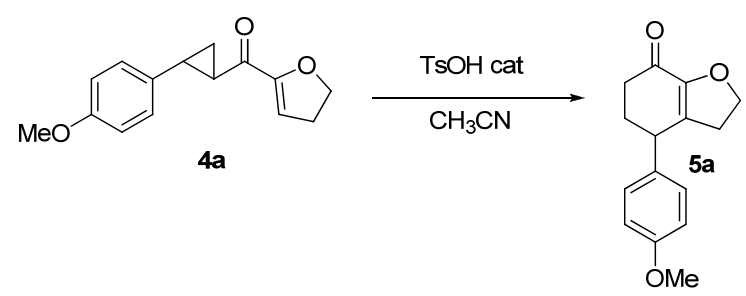

The reaction was performed following general procedure 5, starting from cyclopropane derivate 4a (98 $\mathrm{mg}, 0.40 \mathrm{mmol}, 1.00$ equiv) and tosic acid (15 mg, $0.080 \mathrm{mmol}, 0.20$ equiv). The reaction was quenched after $36 \mathrm{~h}$. Purification by flash chromatography (PET/AcOEt, 7:3) afforded 5a (15 mg, $0.060 \mathrm{mmol}, 15 \%$ ) as yellow oil. $R_{f} 0.40$ (PET/AcOEt 7:3, Anisaldehyde). ${ }^{1} \mathrm{H} \mathrm{NMR}(\mathrm{CDCl} 3,400 \mathrm{MHz}) \delta 7.12(\mathrm{~d}, J=8.5 \mathrm{~Hz}$, $2 \mathrm{H}$; Ar-H), 6.89 (d, J = 8.5 Hz, 2H; Ar-H), $4.50-4.37\left(\mathrm{~m}, 2 \mathrm{H} ; \mathrm{CH}_{2} \mathrm{O}\right), 3.81\left(\mathrm{~s}, 3 \mathrm{H} ; \mathrm{OCH}_{3}\right), 3.75(\mathrm{~m}, 1 \mathrm{H}$; $\mathrm{CH}$ Ar), $2.65\left(\mathrm{t}, J=9.6 \mathrm{~Hz}, 2 \mathrm{H} ; \mathrm{CH}_{2}\right), 2.59-2.50\left(\mathrm{~m}, 1 \mathrm{H} ; \mathrm{CH}_{2}\right), 2.49-2.31\left(\mathrm{~m}, 2 \mathrm{H} ; \mathrm{CH}_{2}\right), 2.11-1.98(\mathrm{~m}$, $\left.1 \mathrm{H} ; \mathrm{CH}_{2}\right) .{ }^{13} \mathrm{C} \mathrm{NMR}\left(\mathrm{CDCl}_{3}, 100 \mathrm{MHz}\right) \delta 190.3,158.7,150.2,136.9,133.1,128.6,114.2,69.3,55.3,41.2$, 36.5, 33.1, 32.8. IR v $2951(\mathrm{w}), 2934(\mathrm{w}), 2838(\mathrm{w}), 1767(\mathrm{w}), 1676(\mathrm{~s}), 1641(\mathrm{w}), 1611(\mathrm{~m}), 1512(\mathrm{~s}), 1463$ (w), 1443 (w), 1395 (w), 1340 (w), 1302 (w), 1244 (s), 1178 (m), 1148 (w), 1102 (s), 1033 (m), 1003 (m), $935(\mathrm{w}), 874(\mathrm{w}), 834(\mathrm{~m}), 771(\mathrm{w}), 736(\mathrm{w})$. HRMS(ESI) calcd for $\mathrm{C}_{15} \mathrm{H}_{16} \mathrm{O}_{3}{ }^{+}(\mathrm{M}+\mathrm{H})$ 245.1172, found 245.1171 .

4-(2,4-Dimethoxyphenyl)-2,3,5,6-tetrahydrobenzofuran-7(4H)-one (5b)

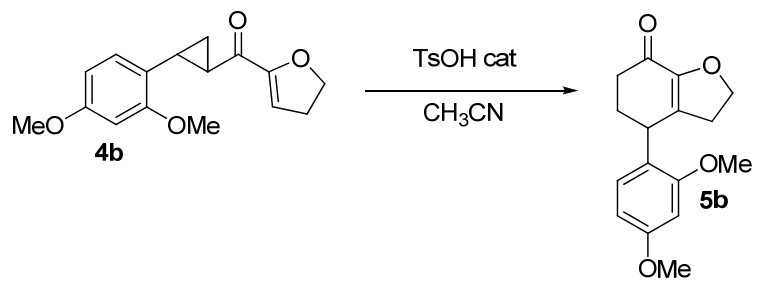

The reaction was carried out following general procedure 6 from cyclopropane $\mathbf{4 b}$ (110 $\mathrm{mg}, 0.400 \mathrm{mmol}$, 1.00 equiv) and tosic acid ( $15 \mathrm{mg}, 0.080 \mathrm{mmol}, 0.20$ equiv). The reaction was quenched after $15 \mathrm{~min}$ to give 5b $(104 \mathrm{mg}, 0.380 \mathrm{mmol}, 95 \%)$ without further purification as yellow oil. $R_{f} 0.30$ (PET/AcOEt 7:3, Anisaldehyde). ${ }^{1} \mathrm{H}$ NMR $\left(\mathrm{CDCl}_{3}, 400 \mathrm{MHz}\right) \delta 6.95$ (d, $\left.J=8.3 \mathrm{~Hz}, 1 \mathrm{H} ; \mathrm{Ar}-\mathrm{H}\right), 6.49$ (s, 1H; Ar-H), 6.45 (d, $J$ $=8.3 \mathrm{~Hz}, 1 \mathrm{H} ; \mathrm{Ar}-\mathrm{H}), 4.42\left(\mathrm{td}, J=3.0,9.6 \mathrm{~Hz}, 2 \mathrm{H} ; \mathrm{CH}_{2} \mathrm{O}\right), 4.13(\mathrm{dd}, J=5.2,6.4 \mathrm{~Hz}, 1 \mathrm{H} ; \mathrm{CH} \mathrm{Ar}), 3.82(\mathrm{~s}$, $\left.3 \mathrm{H} ; \mathrm{OCH}_{3}\right), 3.80$ (s, 3H; $\left.\mathrm{OCH}_{3}\right), 2.67$ (t, $\left.J=9.6 \mathrm{~Hz}, 2 \mathrm{H} ; \mathrm{CH}_{2}\right), 2.53-2.34\left(\mathrm{~m}, 2 \mathrm{H} ; \mathrm{CH}_{2}\right), 2.27(\mathrm{~d}, J=4.9$

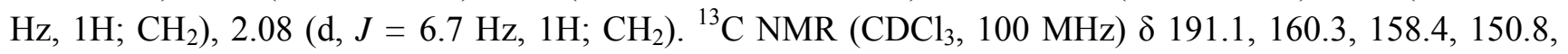
138.1, 128.8, 121.2, 104.4, 99.3, 69.6, 55.7, 55.7, 36.7, 34.9, 33.2, 31.1. IR v $2958(\mathrm{w}), 2838(\mathrm{w}), 1674(\mathrm{~m})$, $1612(\mathrm{~m}), 1587$ (m), $1506(\mathrm{~m}), 1466(\mathrm{~m}), 1439(\mathrm{~m}), 1419(\mathrm{~m}), 1293(\mathrm{~m}), 1262(\mathrm{~m}), 1208(\mathrm{~m}), 1159(\mathrm{~m})$, $1102(\mathrm{~m}), 1035$ (m), 906 (s), 839 (m), 726 (s), $648(\mathrm{~m})$. HRMS(ESI) calcd for $\mathrm{C}_{16} \mathrm{H}_{18} \mathrm{O}_{4}{ }^{+}(\mathrm{M}+\mathrm{H})$ 275.1278, found 275.1292 .

\section{4-(4-Methoxyphenyl)-9-methyl-2,3,4,9-tetrahydro-1H-carbazol-1-one (7)}

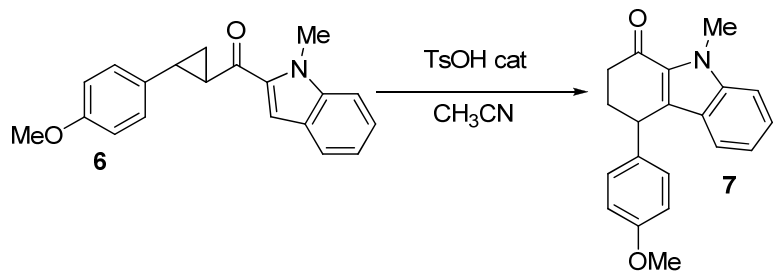

Following general procedure 5, tosic acid ( $15 \mathrm{mg}, 0.08 \mathrm{mmol}, 0.2$ equiv) was added to a cyclopropane 6 (122 mg, $0.400 \mathrm{mmol}, 1.00$ equiv). The reaction was quenched after $3 \mathrm{~h}$ and $30 \mathrm{~min}$ to give 7 (122 $\mathrm{mg}, 0.400$ 
mmol, $100 \%$ ) as a yellow oil without further purification. $\boldsymbol{R}_{\boldsymbol{f}} 0.30$ (PET/AcOEt 8:2, Anisaldehyde). ${ }^{1} \mathrm{H}$ NMR $\left(\mathrm{CDCl}_{3}, 400 \mathrm{MHz}\right) \delta 7.38-7.30(\mathrm{~m}, 2 \mathrm{H} ; \mathrm{Ar}-\mathrm{H}), 7.13$ (d, J = 8.6 Hz, 2H; Ar-H), 7.00 - 6.90 (m, 2H; Ar-H), $6.84(\mathrm{~d}, J=8.6 \mathrm{~Hz}, 2 \mathrm{H} ; \mathrm{Ar}-\mathrm{H}), 4.45$ (dd, $J=4.9,7.5 \mathrm{~Hz}, 1 \mathrm{H} ; \mathrm{CH} \mathrm{Ar}), 4.13$ (s, 3H; $\left.\mathrm{NCH}_{3}\right), 3.80$ (s, 3H; $\left.\mathrm{OCH}_{3}\right), 2.76-2.56\left(\mathrm{~m}, 2 \mathrm{H} ; \mathrm{CH}_{2}\right), 2.51$ (ddd, $\left.J=4.6,9.2,12.3 \mathrm{~Hz}, 1 \mathrm{H} ; \mathrm{CH}_{2}\right), 2.25$ (ddd, $J=4.5,11.1,17.4$ $\left.\mathrm{Hz}, 1 \mathrm{H} ; \mathrm{CH}_{2}\right) .{ }^{13} \mathrm{C} \mathrm{NMR}\left(\mathrm{CDCl}_{3}, 100 \mathrm{MHz}\right) \delta 192.7,158.8,140.3,135.9,131.1,131.0,129.5,126.9,124.9$, 122.9, 120.5, 114.3, 110.6, 55.7, 40.1, 38.4, 35.2, 32.0. IR v $3058(\mathrm{w}), 2938(\mathrm{w}), 2833(\mathrm{w}), 1654(\mathrm{~s}), 1612$ (m), 1510 (s), 1471 (m), 1429 (w), 1410 (w), 1375 (w), 1347 (w), 1243 (s), 1203 (w), 1176 (m), 1072 (w), 1035 (m), 909 (m), 833 (m), 730 (s), 648 (w). HRMS(ESI) calcd for $\mathrm{C}_{20} \mathrm{H}_{19} \mathrm{NO}_{2}{ }^{+}(\mathrm{M}+\mathrm{H})$ 306.1489, found 306.1478 .

4-(2,4-Dimethoxyphenyl)-3,4,4a,5,6,7-hexahydronaphthalen-1(2H)-one (12)

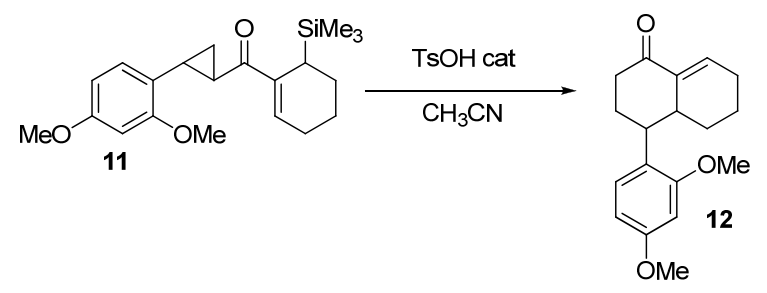

The reaction was performed following general procedure 6, starting from cyclopropane 11 (143 mg, $0.400 \mathrm{mmol}, 1.00$ equiv) and tosic acid (15 $\mathrm{mg}, 0.080 \mathrm{mmol}, 0.20$ equiv). The reaction was quenched after 25 minutes. Purification by flash chromatography (PET/AcOEt, 8:2) afforded 12 (63 mg, $0.22 \mathrm{mmol}, 55 \%$ ) as colorless oil.

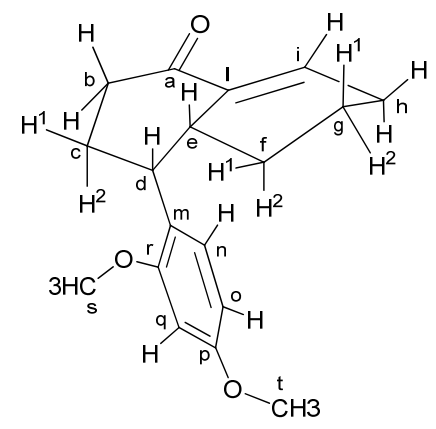

$R_{f} 0.30$ (PET/AcOEt 8:2, Anisaldehyde). ${ }^{1} \mathrm{H}$ NMR $\left(\mathrm{CDCl}_{3}, 400 \mathrm{MHz}\right) \delta 7.06\left(\mathrm{~d}, J=8.0 \mathrm{~Hz}, 1 \mathrm{H} ; \mathrm{H}^{\mathrm{n}}\right), 6.79$ (br s, $\left.1 \mathrm{H} ; \mathrm{H}^{\mathrm{i}}\right), 6.48\left(\mathrm{~d}, J=7.7 \mathrm{~Hz}, 2 \mathrm{H} ; \mathrm{H}^{\mathrm{l}}\right.$ and $\left.\mathrm{H}^{\mathrm{m}}\right), 3.81\left(\mathrm{~s}, 3 \mathrm{H} ; \mathrm{H}^{\mathrm{s}}\right.$ or $\left.\mathrm{H}^{\mathrm{t}}\right), 3.80\left(\mathrm{~s}, 3 \mathrm{H} ; \mathrm{H}^{\mathrm{s}}\right.$ or $\left.\mathrm{H}^{\mathrm{t}}\right), 3.03(\mathrm{~m}, 1 \mathrm{H}$; $\left.\mathrm{H}^{\mathrm{d}}\right), 2.74-2.60\left(\mathrm{~m}, 1 \mathrm{H} ; \mathrm{H}^{\mathrm{b}}\right), 2.61-2.54\left(\mathrm{~m}, 1 \mathrm{H} ; \mathrm{H}^{\mathrm{e}}\right), 2.54-2.40\left(\mathrm{~m}, 1 \mathrm{H} ; \mathrm{H}^{\mathrm{b}}\right), 2.20\left(\mathrm{~m}, 2 \mathrm{H} ; \mathrm{H}^{\mathrm{h}}\right), 2.04(\mathrm{~m}$, $\left.1 \mathrm{H} ; \mathrm{H}^{\mathrm{c} 1}\right), 2.01-1.90\left(\mathrm{~m}, 1 \mathrm{H} ; \mathrm{H}^{\mathrm{c} 2}\right), 1.67\left(\mathrm{~m}, 1 \mathrm{H} ; \mathrm{H}^{\mathrm{g} 2}\right), 1.61-1.47\left(\mathrm{~m}, 1 \mathrm{H} ; \mathrm{H}^{\mathrm{f} 2}\right), 1.36\left(\mathrm{~m}, 1 \mathrm{H} ; \mathrm{H}^{\mathrm{g} 1}\right), 1.10(\mathrm{~m}$, $\left.1 \mathrm{H} ; \mathrm{H}^{\mathrm{fl}}\right) .{ }^{13} \mathrm{C}$ NMR $\left(\mathrm{CDCl}_{3}, 100 \mathrm{MHz}\right) \delta 201.1(\mathrm{a}), 159.0(\mathrm{r}), 158.2(\mathrm{p}), 139.7(\mathrm{l}), 136.8(\mathrm{i}), 127.5(\mathrm{n}), 124.8$ (m), 104.3 (o), 98.5 (q), 55.4 (s or t), 55.3 (s or t), 42.4 (d), 40.4 (e), 39.2 (b), 29.9 (c), 27.8 (f), 26.2 (h), 21.4 (g). IR $v 3000(\mathrm{w}), 2935(\mathrm{~m}), 2861(\mathrm{w}), 2836(\mathrm{w}), 1686(\mathrm{~s}), 1613(\mathrm{~s}), 1587(\mathrm{~m}), 1507$ (s), 1465 (m), 1456 (m), $1420(\mathrm{w}), 1328(\mathrm{w}), 1296(\mathrm{~m}), 1268$ (m), 1209 (s), 1158 (m), 1036 (m), 927 (w), $836(\mathrm{w}), 737$ (w). HRMS(ESI) calcd for $\mathrm{C}_{18} \mathrm{H}_{22} \mathrm{O}_{3}{ }^{+}(\mathrm{M}+\mathrm{H})$ 287.1647, found 287.1636.

Further analytical data: COESY, NOESY and HSQC.

Important signal for NOESY : $\mathbf{H}^{\mathrm{e}}-\mathbf{H}^{\mathrm{d}}(\mathbf{s}) ; \mathbf{H}^{\mathrm{e}}-\mathbf{H}^{\mathrm{f} 1}(\mathrm{~m}) ; \mathbf{H}^{\mathrm{d}}-\mathbf{H}^{\mathrm{cl}}(\mathbf{s})$.

1-Cyclohex-1-enyl-4-hydroxy-4-(4-methoxy-phenyl)-butan-1-one (16) 


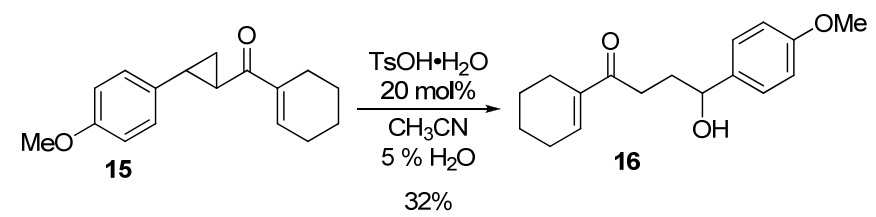

A solution of cyclopropane 15 (81 $\mathrm{mg}, 0.32 \mathrm{mmol}, 1.0$ equiv) and tosic acid monohydrate (59 $\mathrm{mg}, 0.32$ mmol, 1.0 equiv) in acetonitrile $(8 \mathrm{~mL})$ and water $(0.4 \mathrm{~mL})$ was stirred at RT for $36 \mathrm{~h}$. The solution was quenched with $\mathrm{NaHCO}_{3}(10 \mathrm{~mL})$ and extracted with $\mathrm{Et}_{2} \mathrm{O}(3 \times 10 \mathrm{~mL})$. The combined organic layers were washed with brine $(2 \times 10 \mathrm{~mL})$, dried over $\mathrm{MgSO}_{4}$ and the solvent was removed under reduced pressure. Purification by flash chromatography (PET/AcOEt, 4:1-2:1) afforded 16 (27 mg, $0.099 \mathrm{mmol}, 32 \%$ ) as yellow oil. ( $\boldsymbol{R}_{\boldsymbol{f}} 0.20$ (PET/AcOEt 4:1, Anisaldehyde). ${ }^{1} \mathrm{H} \mathrm{NMR}\left(\mathrm{CDCl}_{3}, 400 \mathrm{MHz}\right) \delta$ 7.25-7.30 (m, $2 \mathrm{H}$, Ar$\mathrm{H}), 6.83-6.92(\mathrm{~m}, 3 \mathrm{H}, \mathrm{Ar}-\mathrm{H}$ and alkene-H), 4.69 (br m, $1 \mathrm{H}, \mathrm{CHOH}), 3.80$ (s, $\left.3 \mathrm{H}, \mathrm{OCH}_{3}\right), 2.75$ (td, $J=7.0$, $1.0 \mathrm{~Hz}, 2 \mathrm{H}$, ketone- $\mathrm{CH}_{2}$ ), 2.47 (br s, $\left.1 \mathrm{H}, \mathrm{OH}\right), 2.17-2.29$ (m, $4 \mathrm{H}$, cyclohexene- $\left.\mathrm{CH}_{2}\right), 2.05$ (q, $J=6.9 \mathrm{~Hz}, 2$

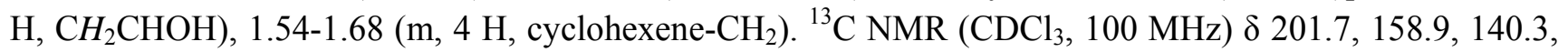
139.0, 136.6, 126.9, 113.8, 73.40, 55.3, 33.4, 33.3., 26.1, 23.1, 21.9, 21.5. IR v 3447 (br w), 2932 (m), 2860 (w), 1663 (m), 1614 (m), 1514 (s), 1458 (w), 1302 (w), 1247 (s), 1175 (m), 1034 (s), 914 (w), 832 (m), 734 (w). HRMS(ESI) calcd for $\mathrm{C}_{17} \mathrm{H}_{22} \mathrm{NaO}_{3}{ }^{+}(\mathrm{M}+\mathrm{Na}) 297.1461$, found 297.1452.

\section{Kinetic Measurements}

\subsection{Methods and Formula}

\section{NMR-Method for the Monitoring of the Formal Homo-Nazarov Cyclization}

Substrate 2a (6.00 mg, $0.00232 \mathrm{mmol}$, 1.00equiv) was dissolved in $\mathrm{CD}_{3} \mathrm{CN}(0.5 \mathrm{~mL})$ at $23{ }^{\circ} \mathrm{C}$ under nitrogen in a NMR tube. A solution of the desired amount of tosic acid in $\mathrm{CD}_{3} \mathrm{CN}(0.15 \mathrm{~mL})$ was then added and the tube put in the spectrometer as fast as possible (time set to zero at this point). The reaction was monitored by ${ }^{1} \mathrm{H}$-NMR at $400 \mathrm{MHz}$. The reaction was examined with $0.1,0.2,0.3$ and 0.4 equiv tosic acid and 0.4 equiv deuterated tosic acid. The decrease of the concentration of 2a was monitored via the decrease of the integral of the alkene proton peak between 6.0383 and $6.0022 \mathrm{ppm}$, using the internal $\mathrm{CH}_{3} \mathrm{CN}$ signal as standard. The concentration of product $3 \mathrm{a}$ was monitored via the increase of the integral of the benzylic proton between 3.6454 and $3.6050 \mathrm{ppm}$. This signal was increase further by a factor 1.33 (part of the signal was dropped in the integration due to impurity interference, the correction factor was obtained by integrating the missing area on a pure sample) and 1.09 (difference of integration between the two observed signal for a separately prepared 1:1 solution). Better results were obtained with the alkene proton, as the baseline was more stable in this region of the spectra. Spectra were taken every $36.5 \mathrm{~s}$ with one single scan.

\section{Statistical Methods and Formula ${ }^{14}$}

\section{Reaction Rate}

The initial rates $r$ of the reactions were determined using standard linear regression programs (Excel) applied on the linear region of the concentration curves $(4.25 \mathrm{~min})$. The standard deviation and the confidence interval of the data were calculated using following formula:

$\left({ }^{14}\right)$ Meister, E. Grundpraktikum Physikalische Chemie; vdf Hochschulverlag AG: Zürich, 2000. 
Standard deviation of the rate:

$$
s_{r}^{2}=\frac{n}{n \cdot \sum_{i=1}^{n} t_{i}^{2}-\left(\sum_{i=1}^{n} t_{i}\right)^{2}} \cdot \sum_{i=1}^{n}\left(c_{i}-I-r \cdot t_{i}\right)^{2} \cdot \frac{1}{n-2}
$$

Confidence interval (95\%) of the rate: $v_{r}=t_{s} \cdot \frac{s_{r}}{\sqrt{n}}$;

Whereas $\mathrm{n}$ is the amount of data points measured, $\mathrm{t}$ is the time of measurement after the addition of acid, $\mathrm{c}$ is the measured concentration of $\mathbf{2 a}, \mathrm{r}$ is the calculated reaction rate, $\mathrm{I}$ is the calculate intercept of the curve, $t_{s}$ is the student-t factor corresponding to $95 \%$ probability and a degree of freedom of $n-2$.

\section{Van't Hoff Equation}

Van't Hoff Equation: $y=\log r=O \cdot \log c+I=a \cdot \log c+b$;

Standard deviation, confidence interval for y values: $s_{y i}=\frac{s_{r i}}{\operatorname{Ln}(10) \cdot r_{i}}, \quad v_{y i}=\frac{v_{r i}}{\operatorname{Ln}(10) \cdot r_{i}}$;

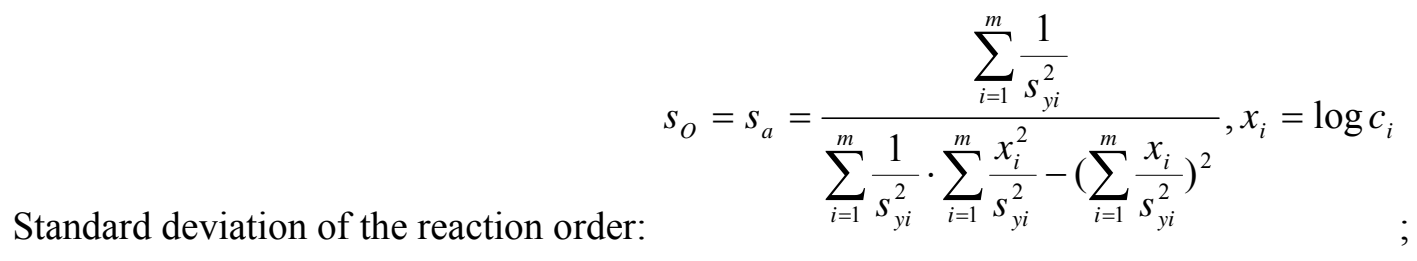

Confidence interval (95\%) of the reaction order: $v_{O}=v_{a}=t_{s} \cdot \frac{s_{a}}{\sqrt{m}}$

Correlation factor R: $\sqrt{\sum_{i=1}^{m} \frac{1}{s_{y i}^{2}} \cdot \sum_{i=1}^{m} \frac{x_{i} y_{i}}{s_{y i}^{2}}-\sum_{i=1}^{m} \frac{x_{i}}{s_{y i}^{2}} \cdot \sum_{i=1}^{m} \frac{y_{i}}{s_{y i}^{2}} \frac{x_{i}^{2}}{s_{y i}^{2}}-\left(\sum_{i=1}^{m} \frac{x_{i}}{s_{y i}^{2}}\right)^{2} \cdot \sqrt{\sum_{i=1}^{m} \frac{1}{s_{y i}^{2}} \cdot \sum_{i=1}^{m} \frac{y_{i}^{2}}{s_{y i}^{2}}-\left(\sum_{i=1}^{m} \frac{y_{i}}{s_{y i}^{2}}\right)^{2}}}$

Whereas $r$ is the calculated reaction rate with standard deviation $\mathrm{s}_{\mathrm{r}}$ and confidence interval $\mathrm{v}_{\mathrm{r}}$, $\mathrm{O}$ is the reaction order with standard deviation $\mathrm{s}_{\mathrm{O}}$ and confidence interval $\mathrm{v}_{\mathrm{O}}, \mathrm{c}$ is the concentration of the examined reagent, $\mathrm{m}$ is the amount of data points measured, $\mathrm{R}$ is the correlation factor, $\mathrm{t}_{\mathrm{s}}$ is the student- $\mathrm{t}$ factor corresponding to $95 \%$ probability and a degree of freedom of $n-2$.

\subsection{Graphical Representation of the Data}




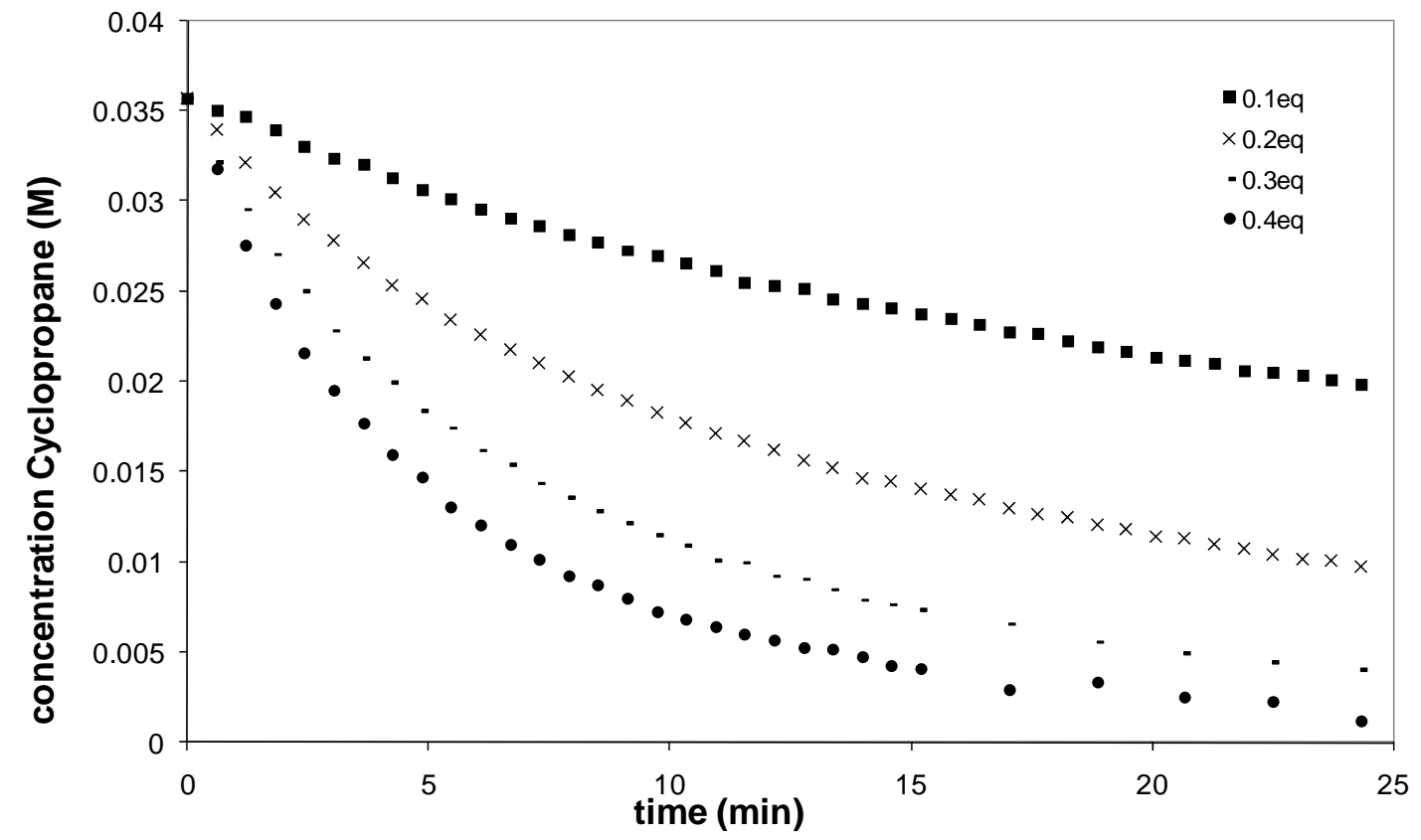

Figure S1. Influence of the concentration of tosic acid on the reaction rate of the starting material 2a.

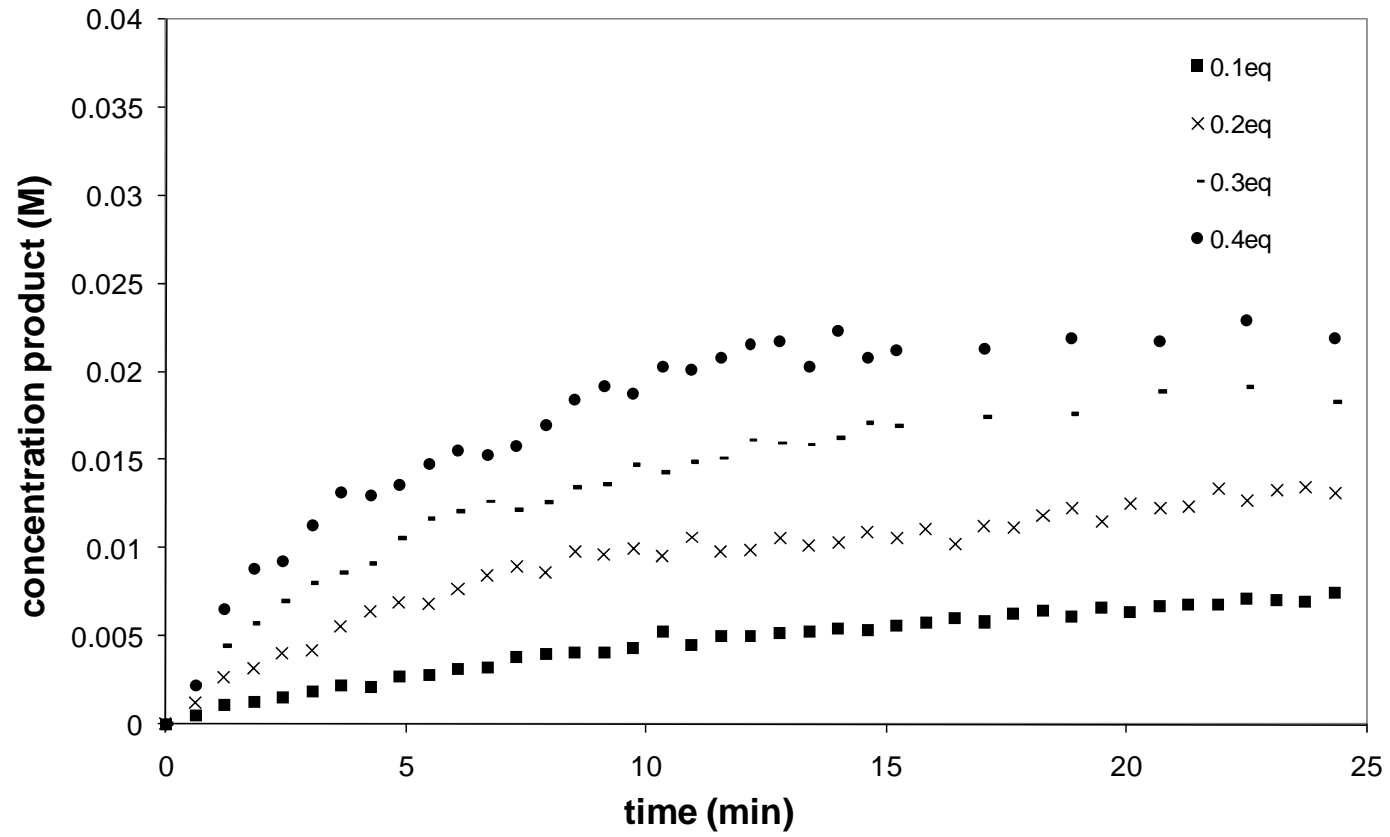

Figure S2. Influence of the concentration of tosic acid on the formation of product 3a. 


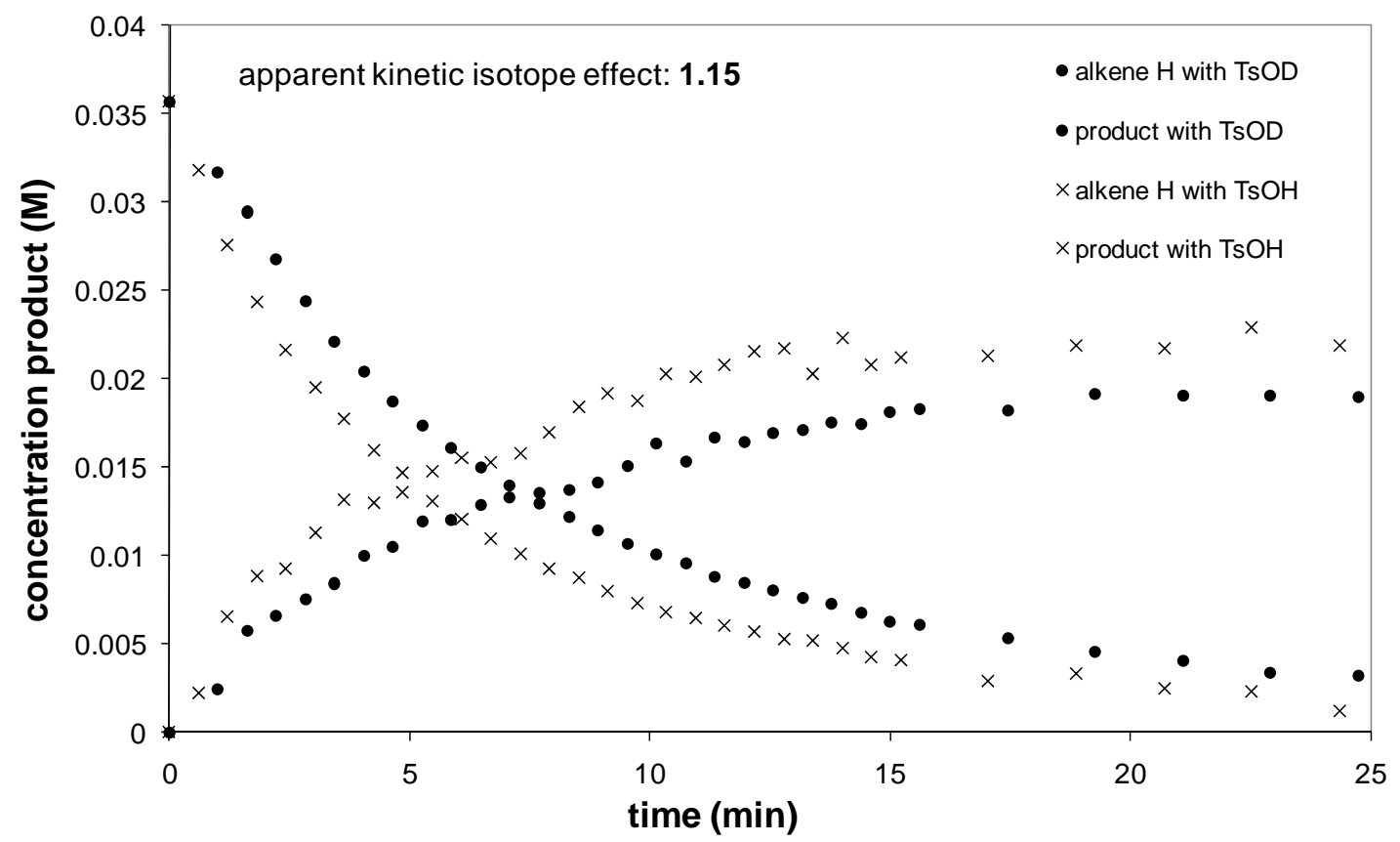

Figure S3. Comparison of tosic acid and deuterated tosic acid: kinetic isotope effect?

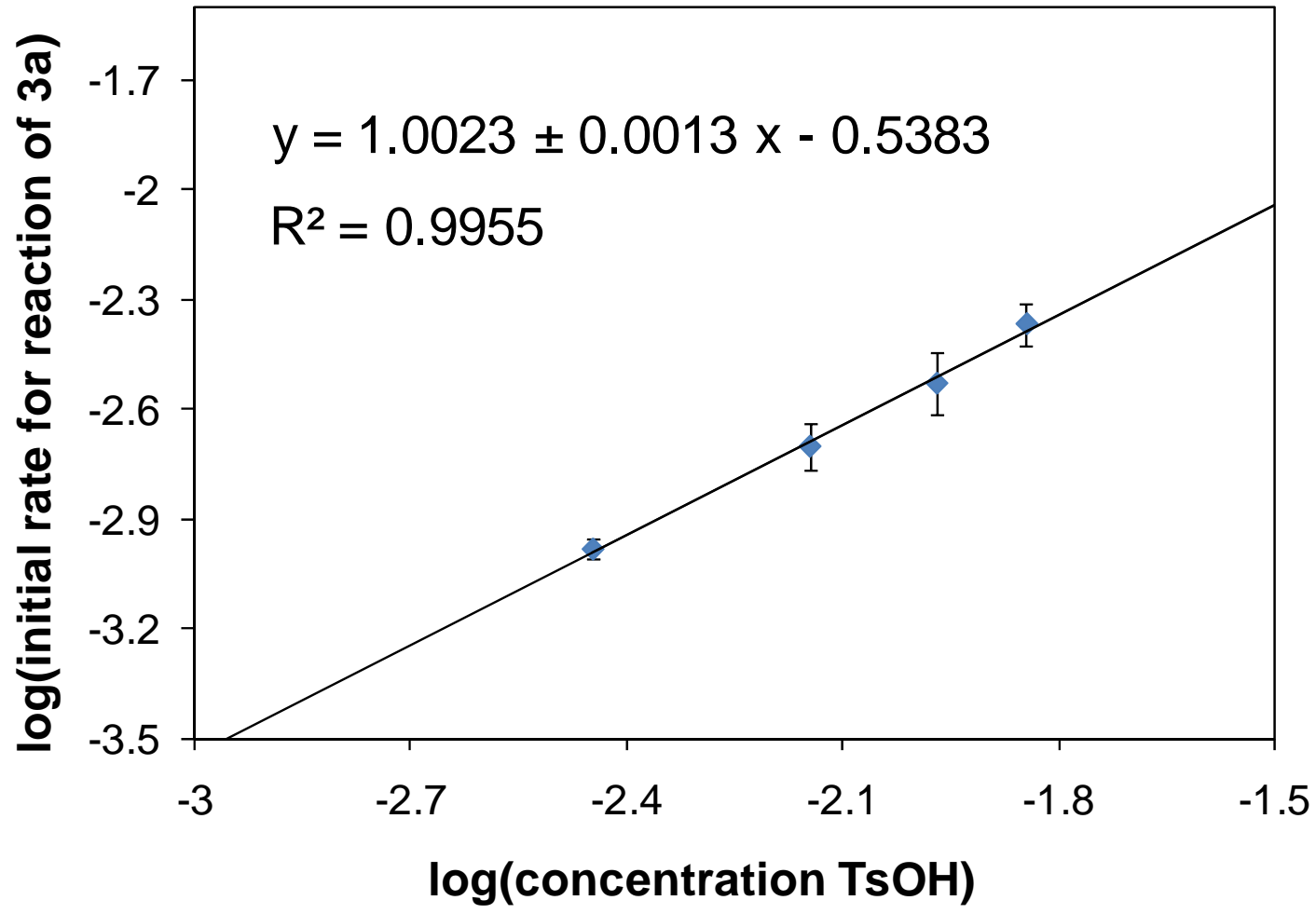

Figure S4. Determination of the rate order using Van't Hoff plot. 
6.1 Important Spectra for Analysis of the Reaction
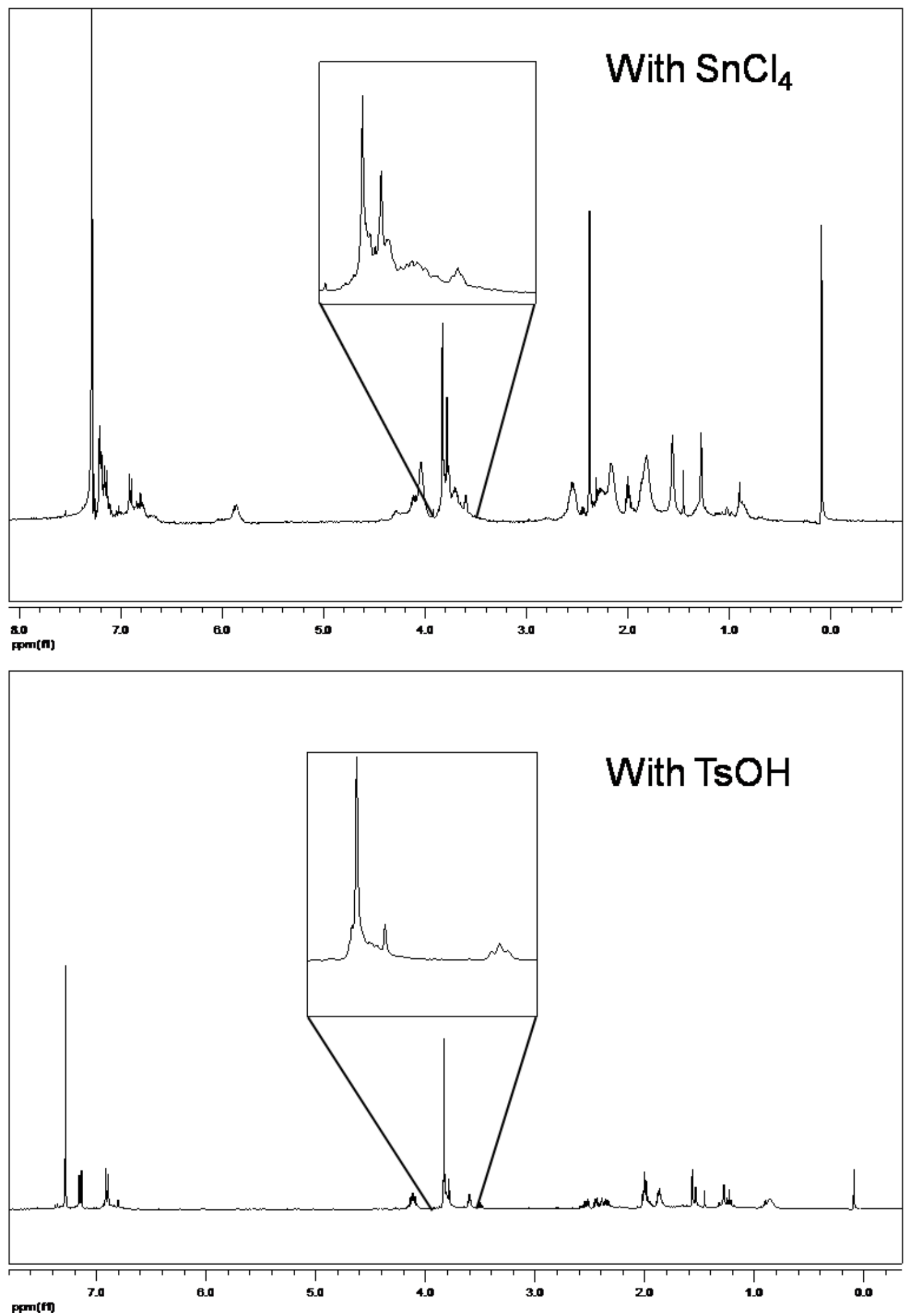

Figure S5. Comparison of the crude NMR of the reaction of $2 \mathbf{a}$ with $\mathrm{SnCl}_{4}$ and $\mathrm{TsOH}$. 

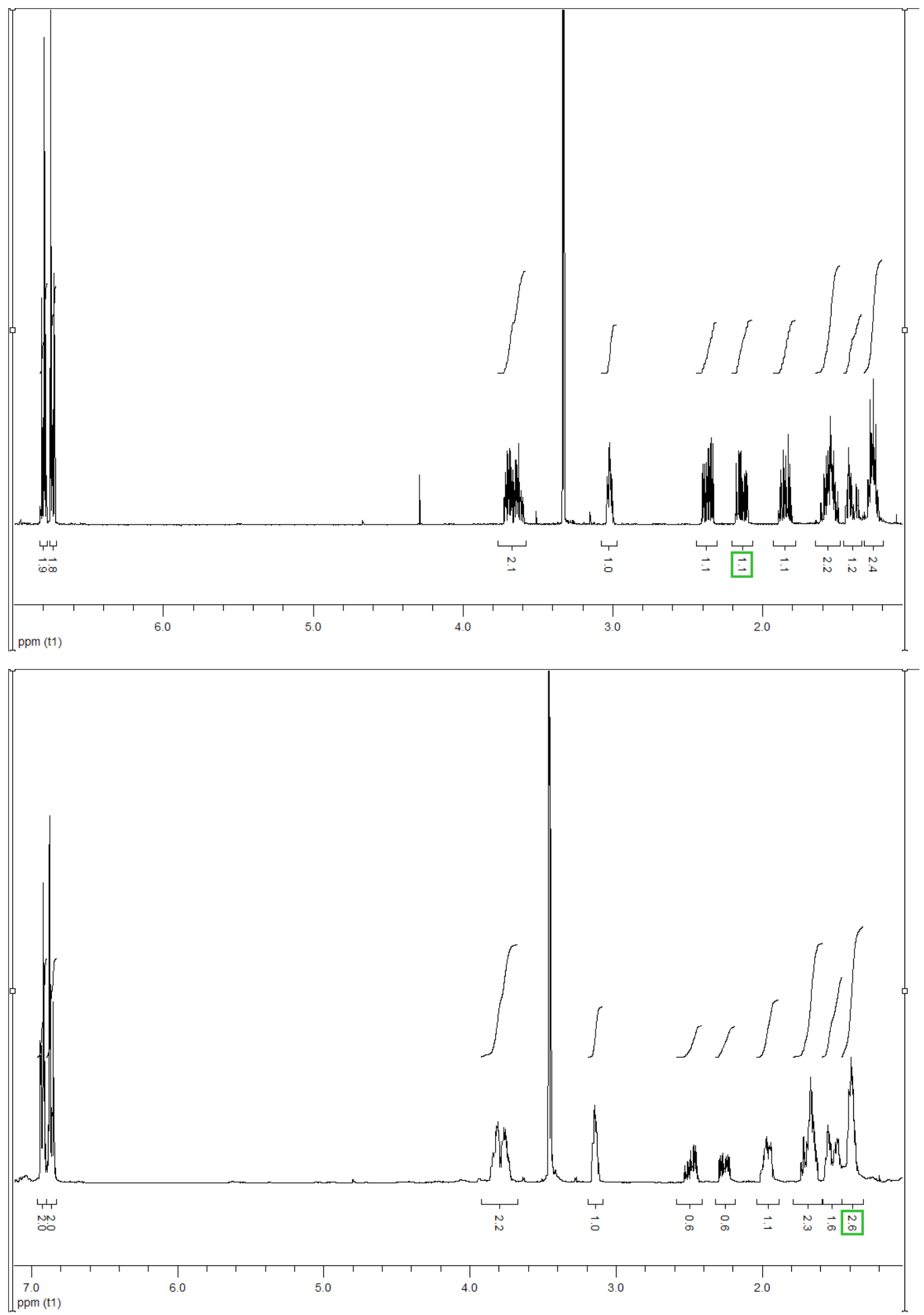

Figure S6 $\quad{ }^{1} \mathrm{H}$ NMR spectra in benzene-d6 of cyclization product 3a obtained using TsOH (top) and TsOD (bottom). 


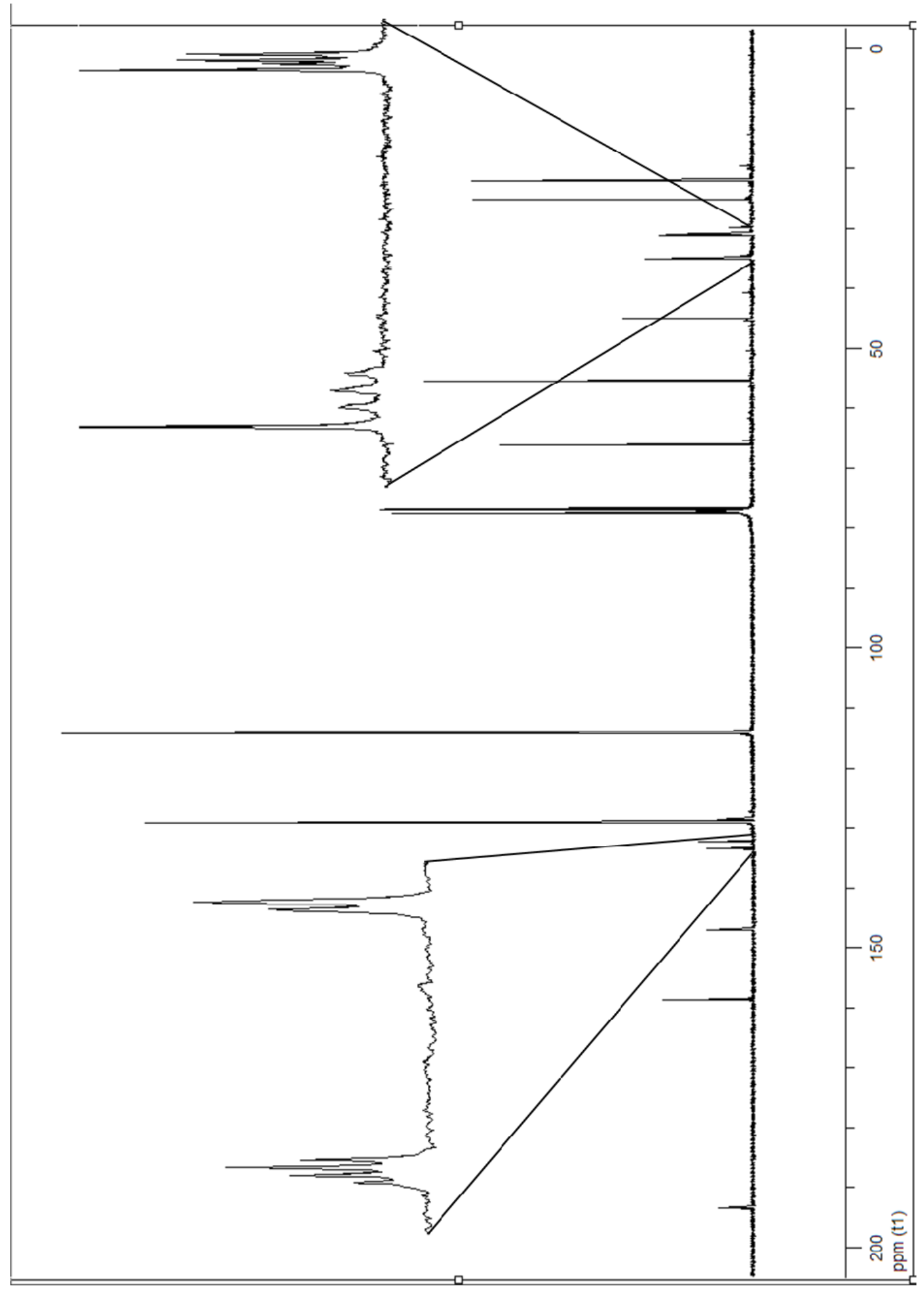

Figure S7 $\quad{ }^{13} \mathrm{C}$ NMR spectra in $\mathrm{CDCl}_{3}$ of cyclization product 3a obtained using TsOD. 


\subsection{Spectra of New Compounds}
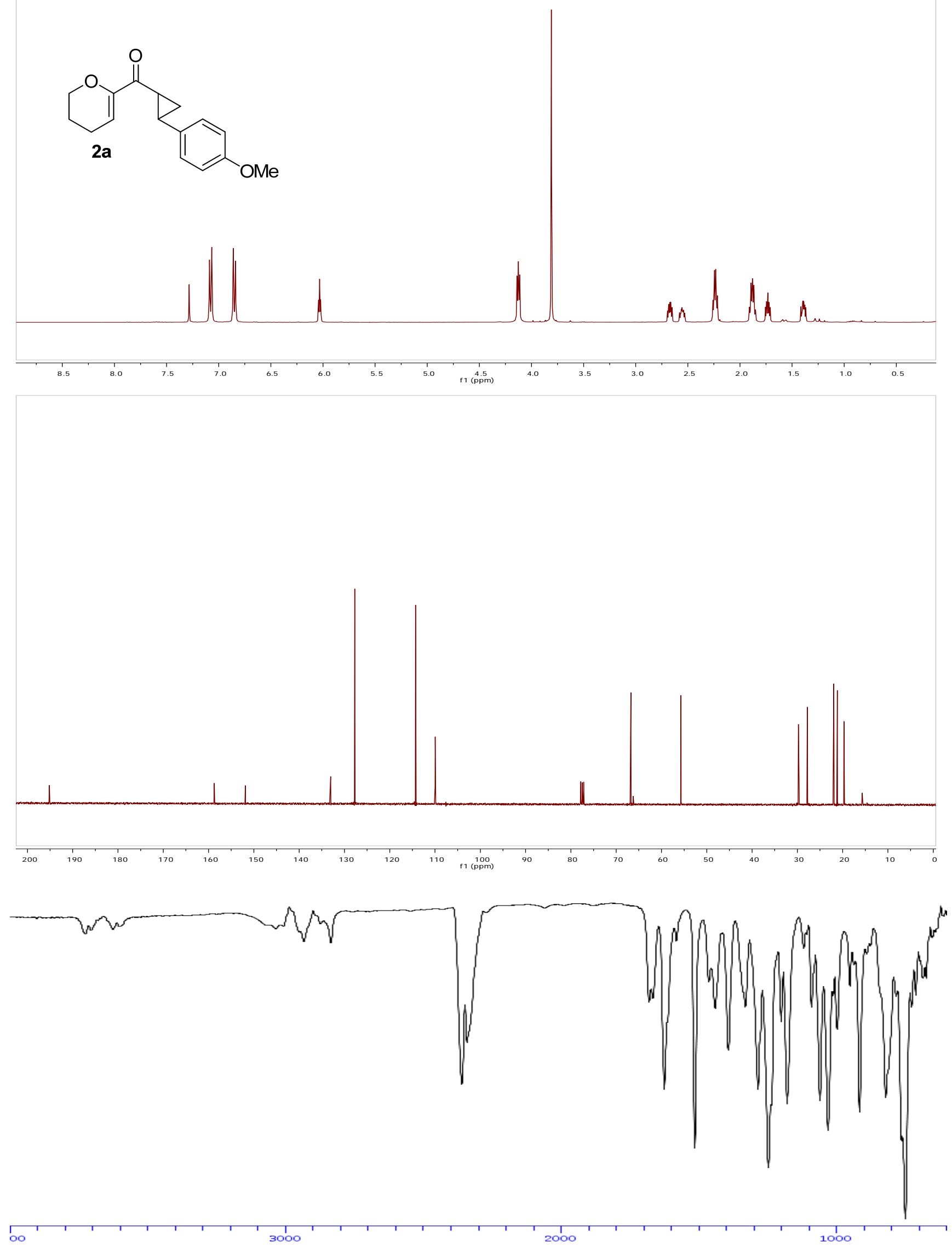
<smiles>O=C(C1=CCCCO1)C1CC1c1ccccc1</smiles>
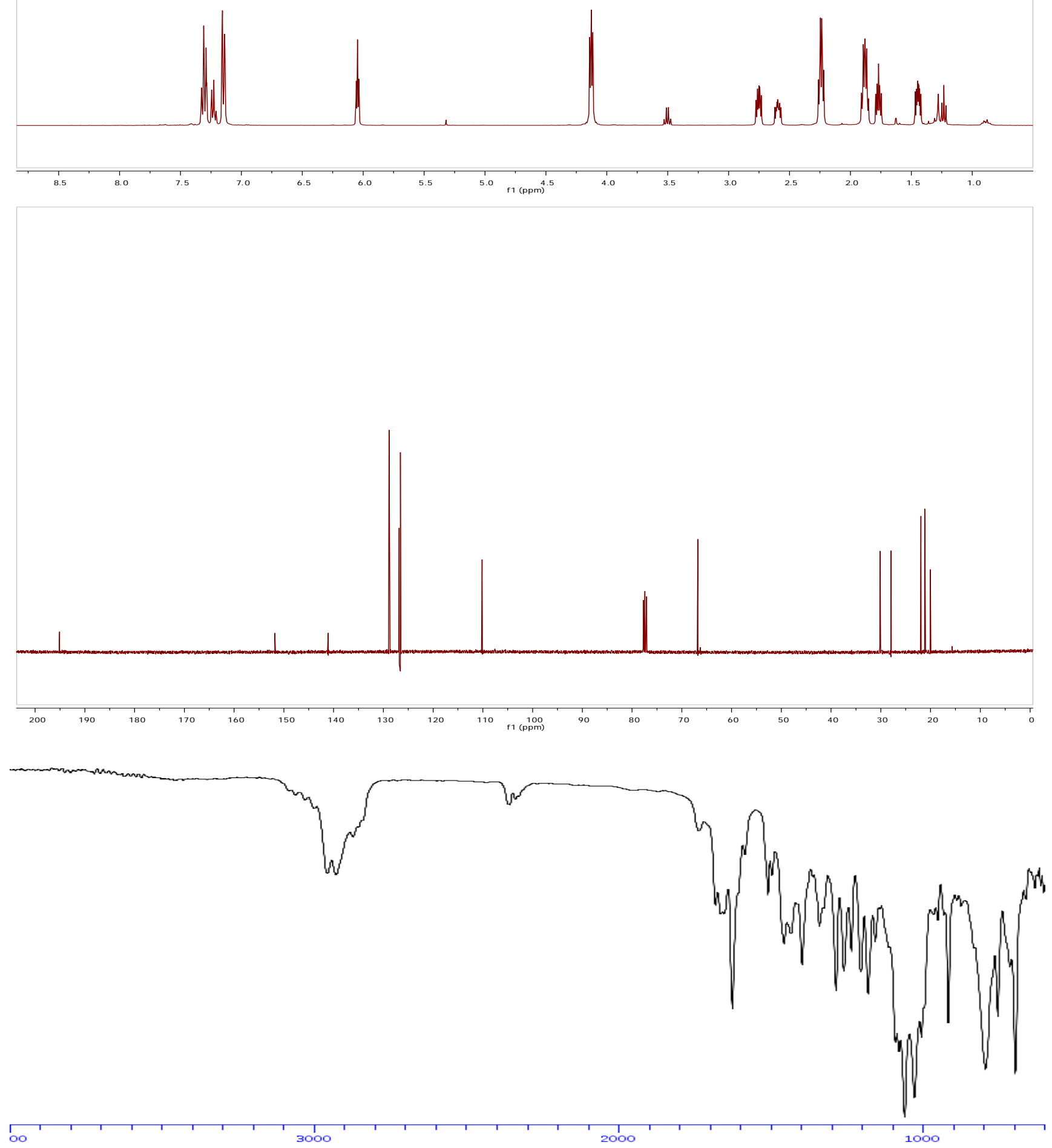

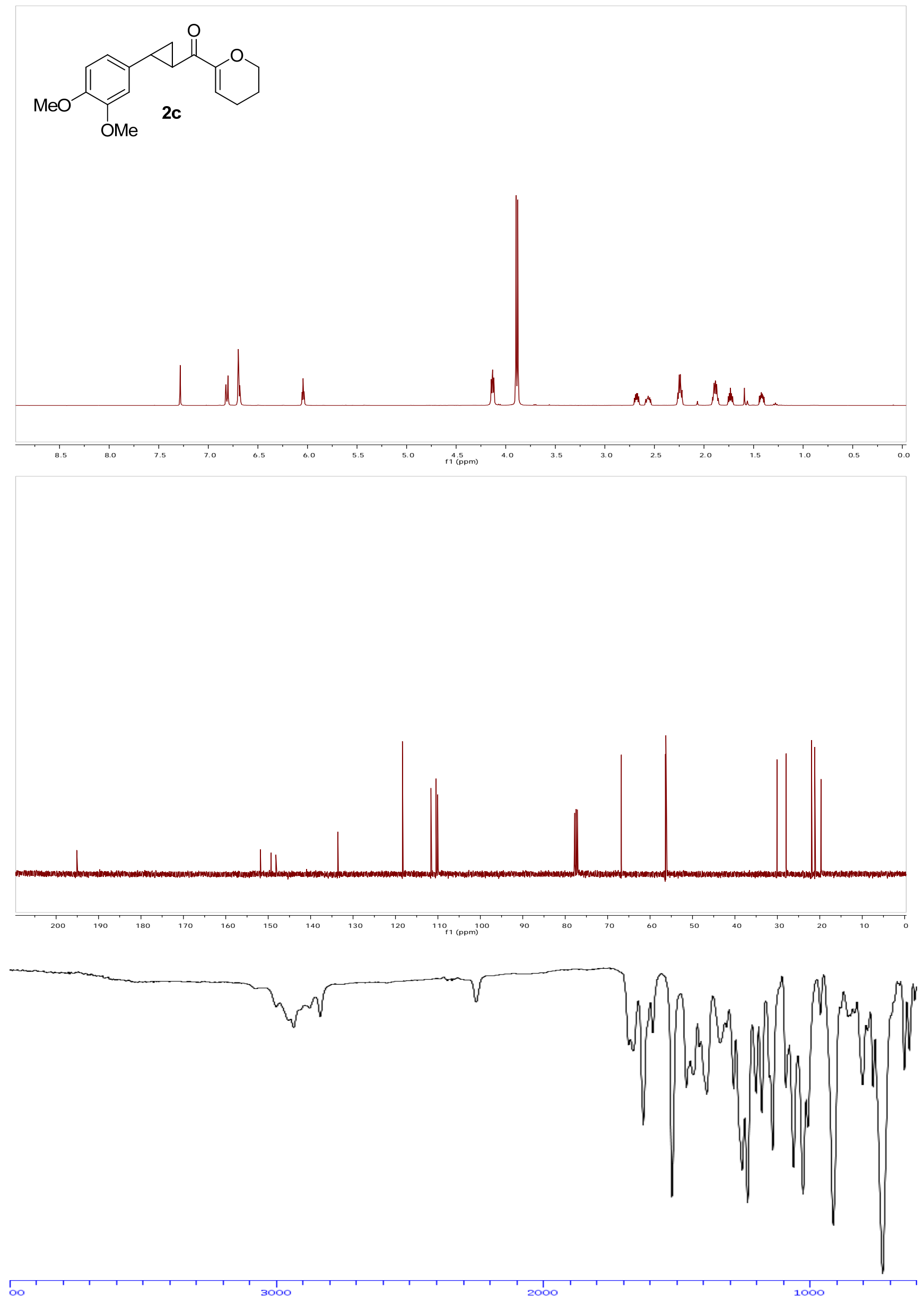

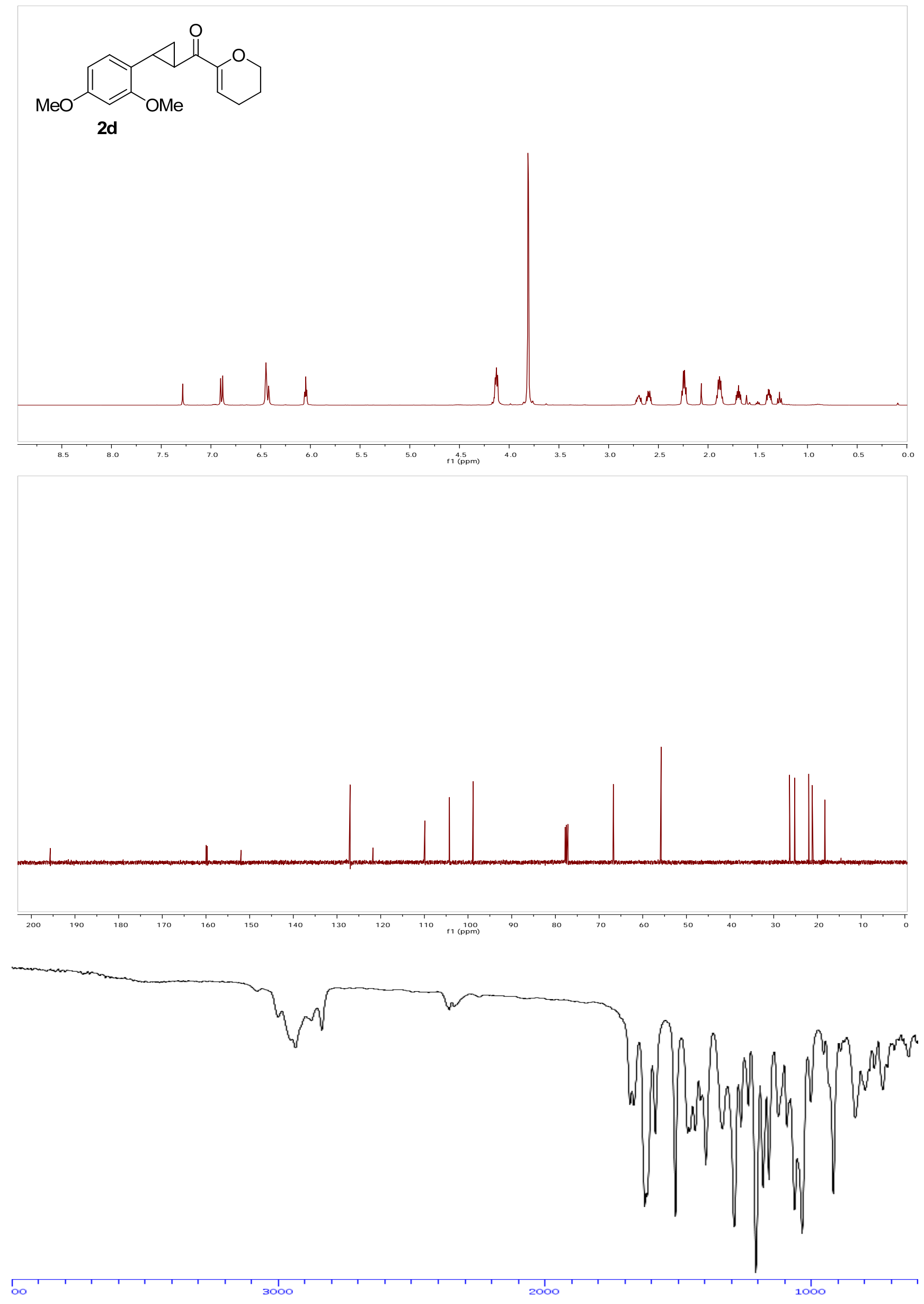


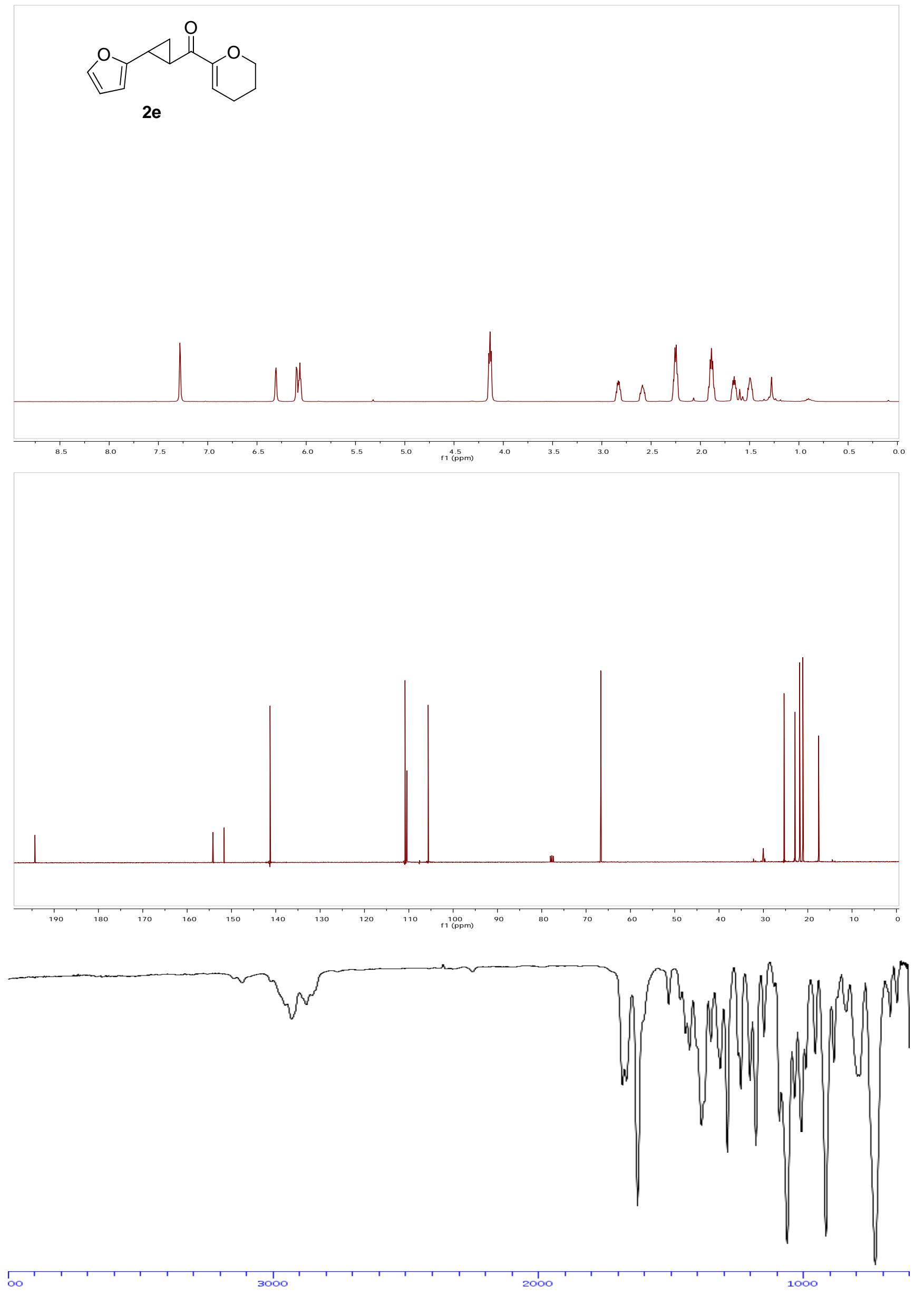



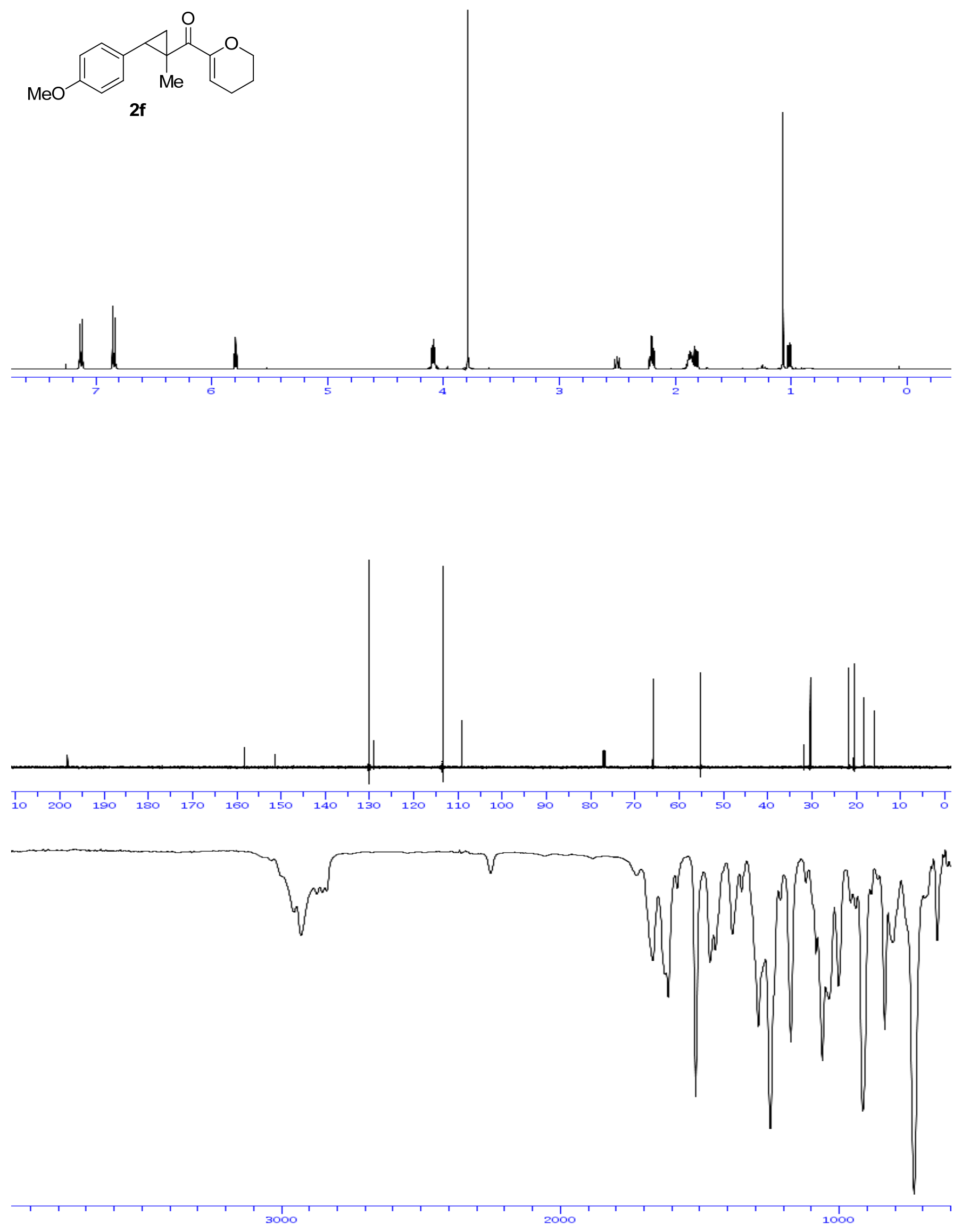

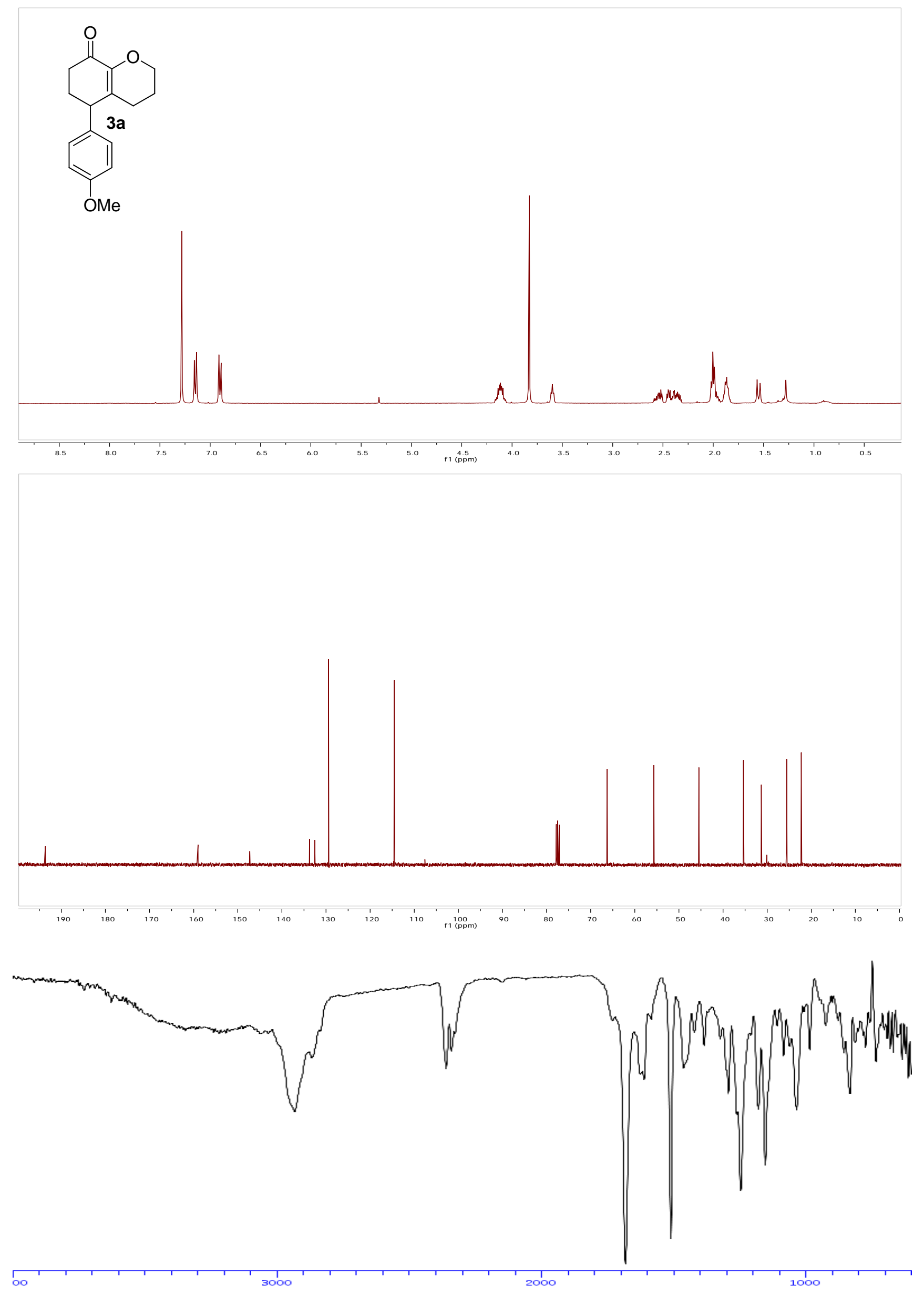

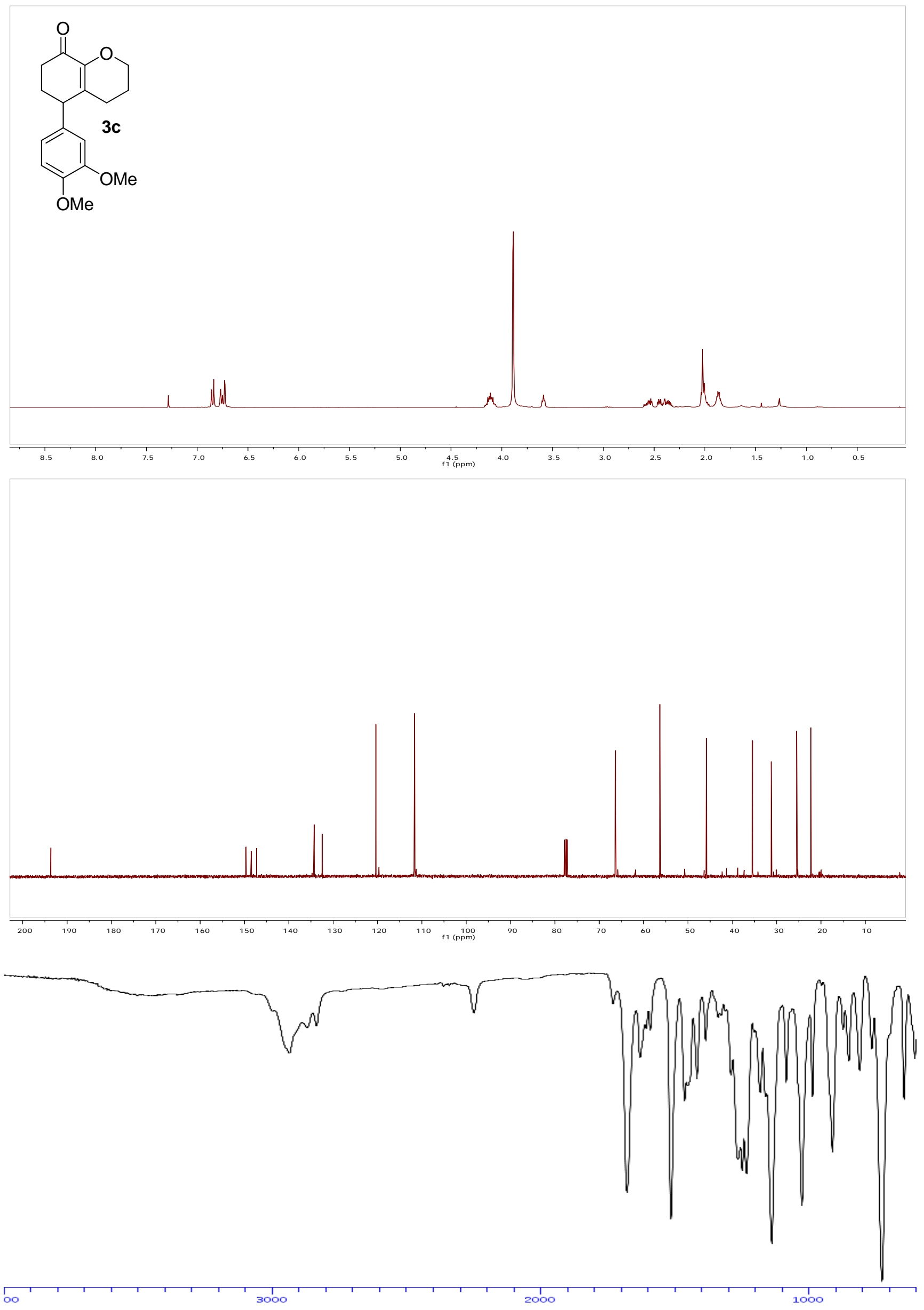

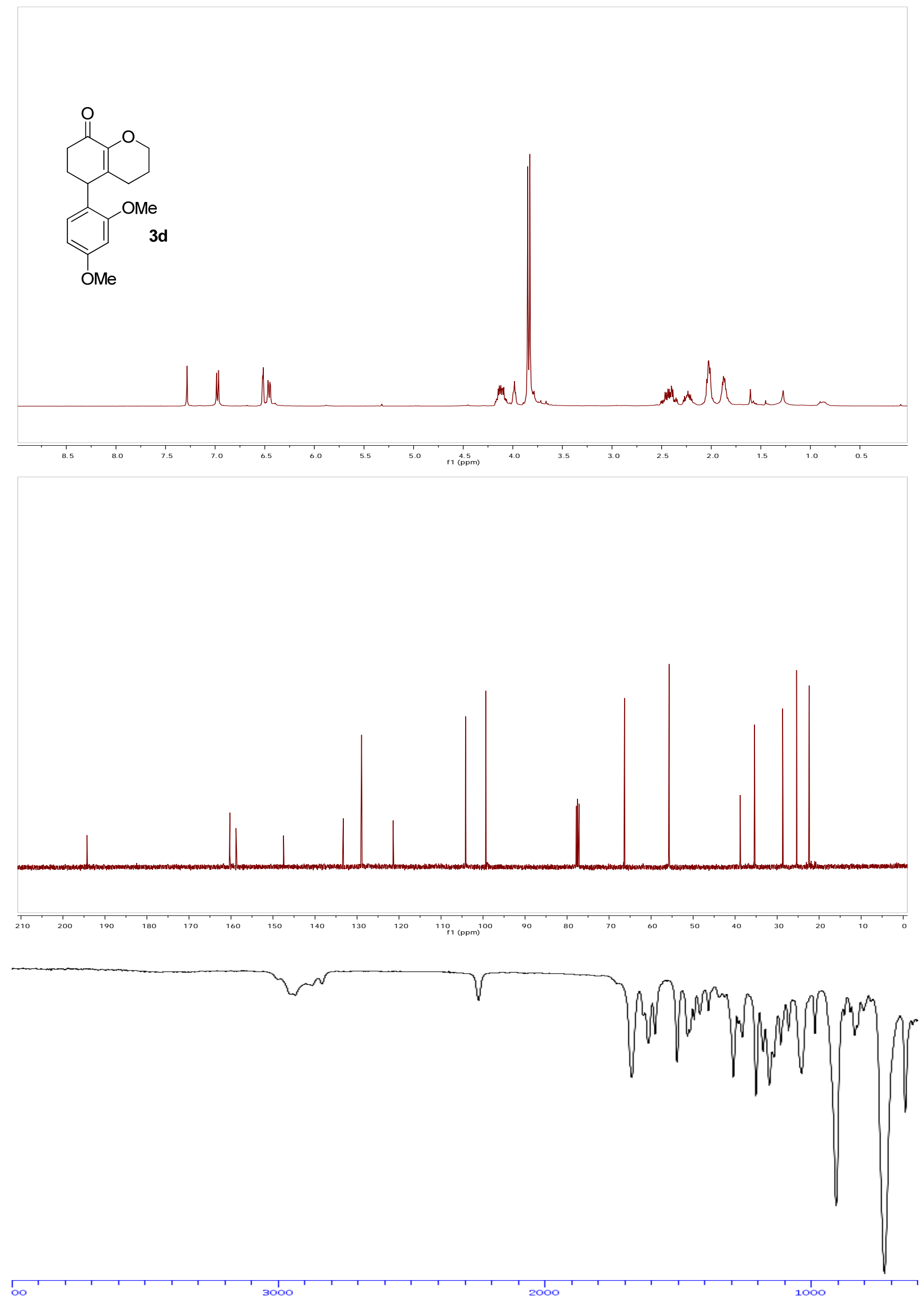

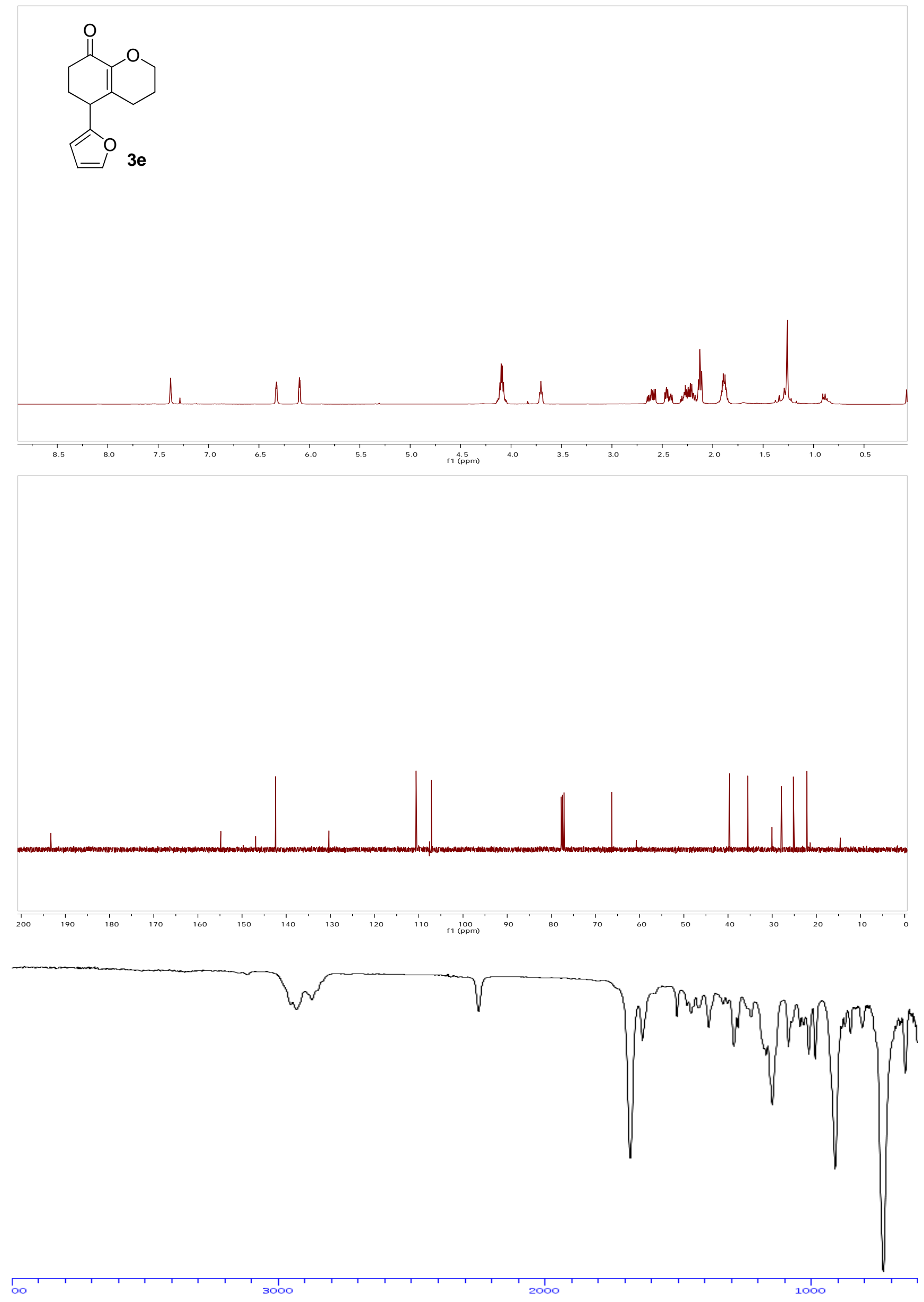

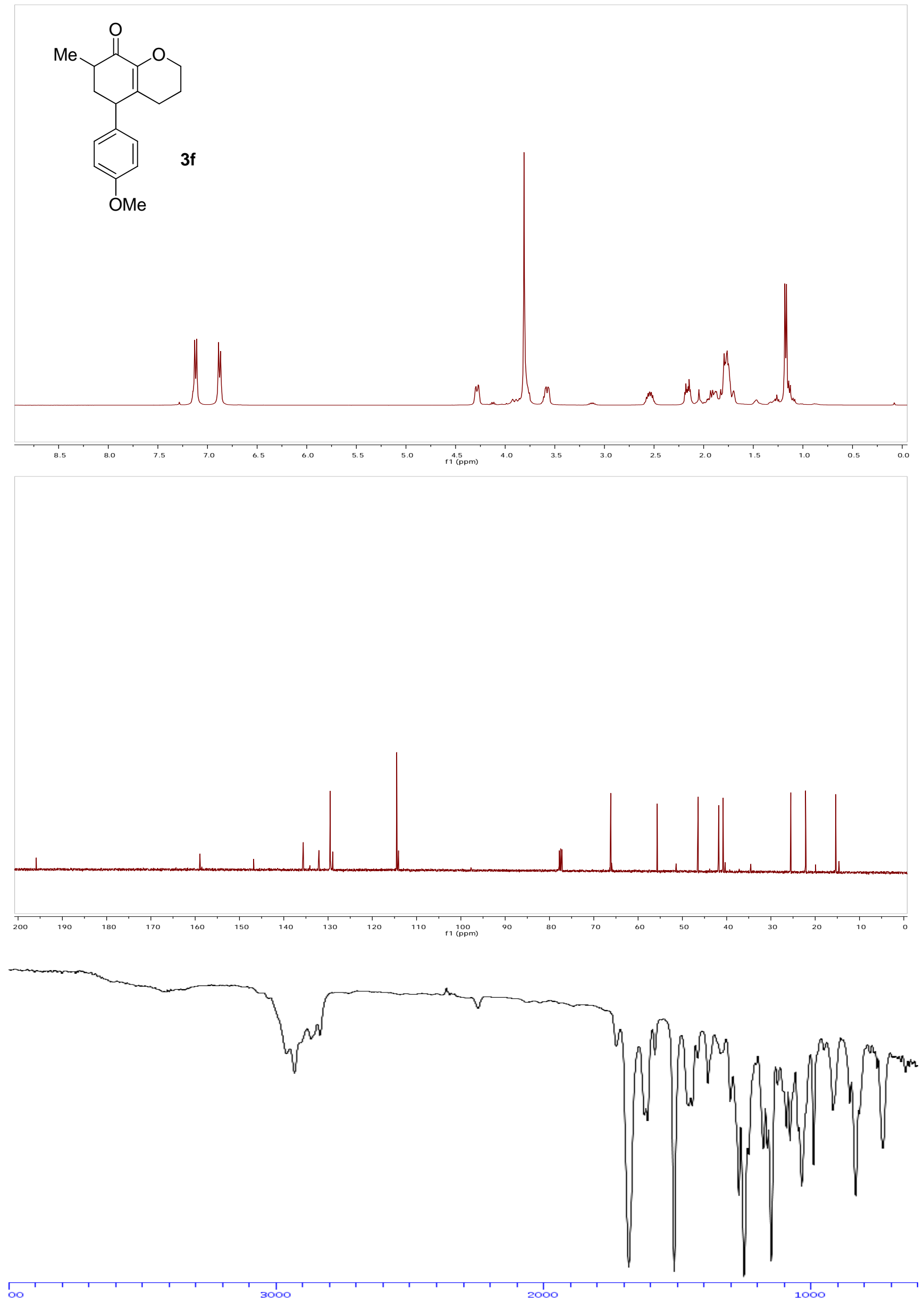
<smiles>COc1ccc(C2CC2C(=O)C2=CCCO2)cc1</smiles>

$4 a$
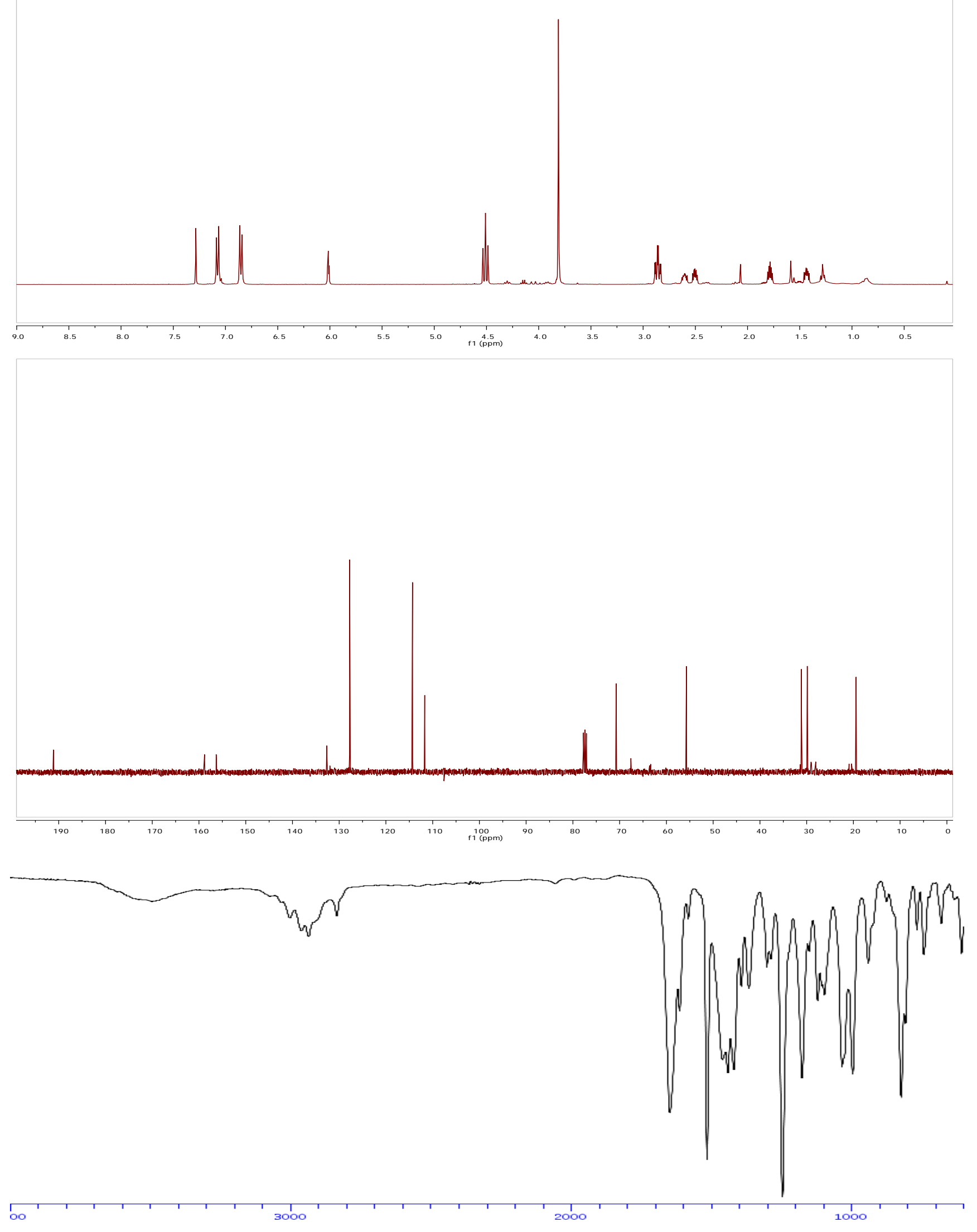

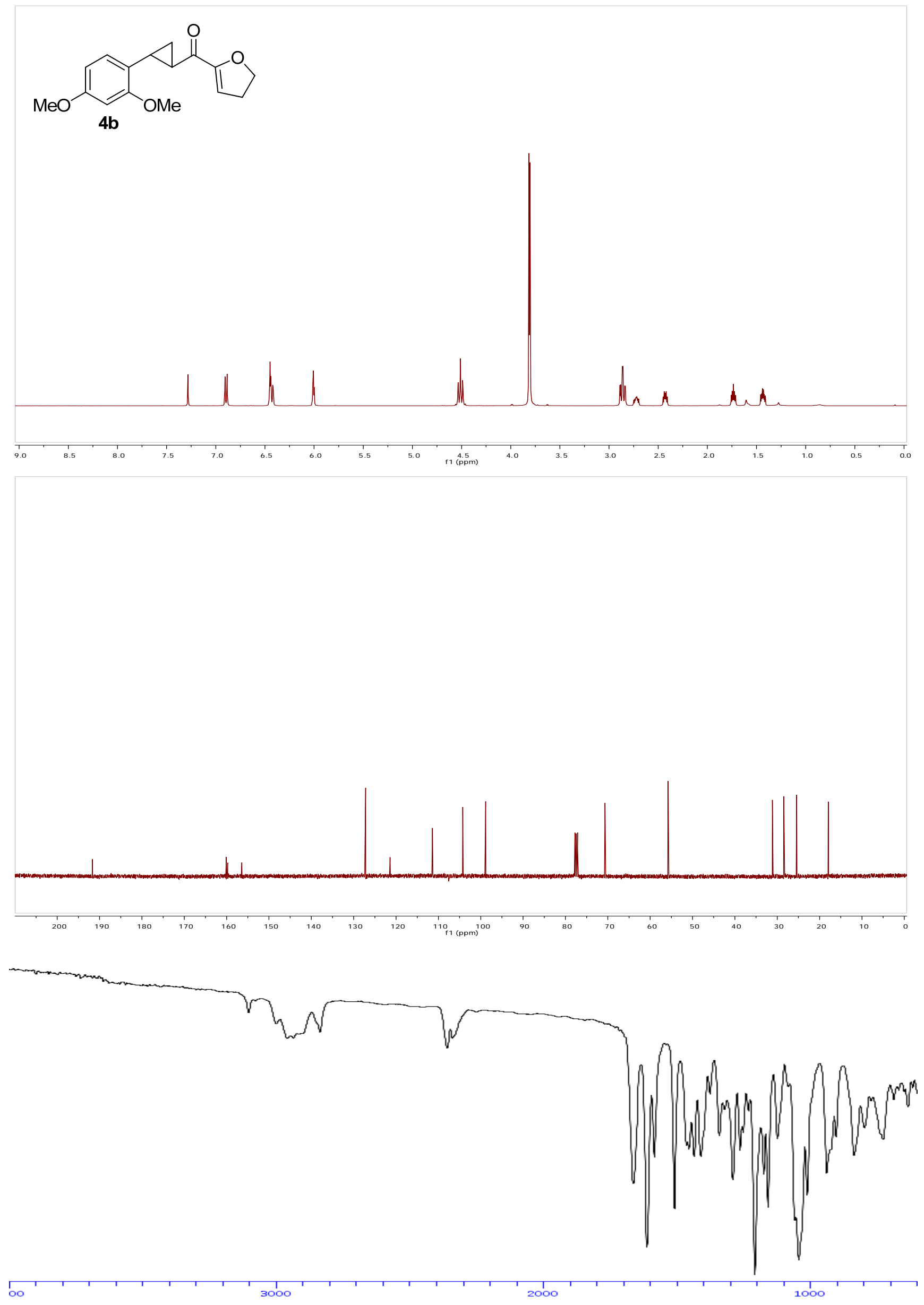
<smiles>O=C1CCC(c2ccc([O-])cc2)C2=C1OCC2</smiles>

all

k

dumener
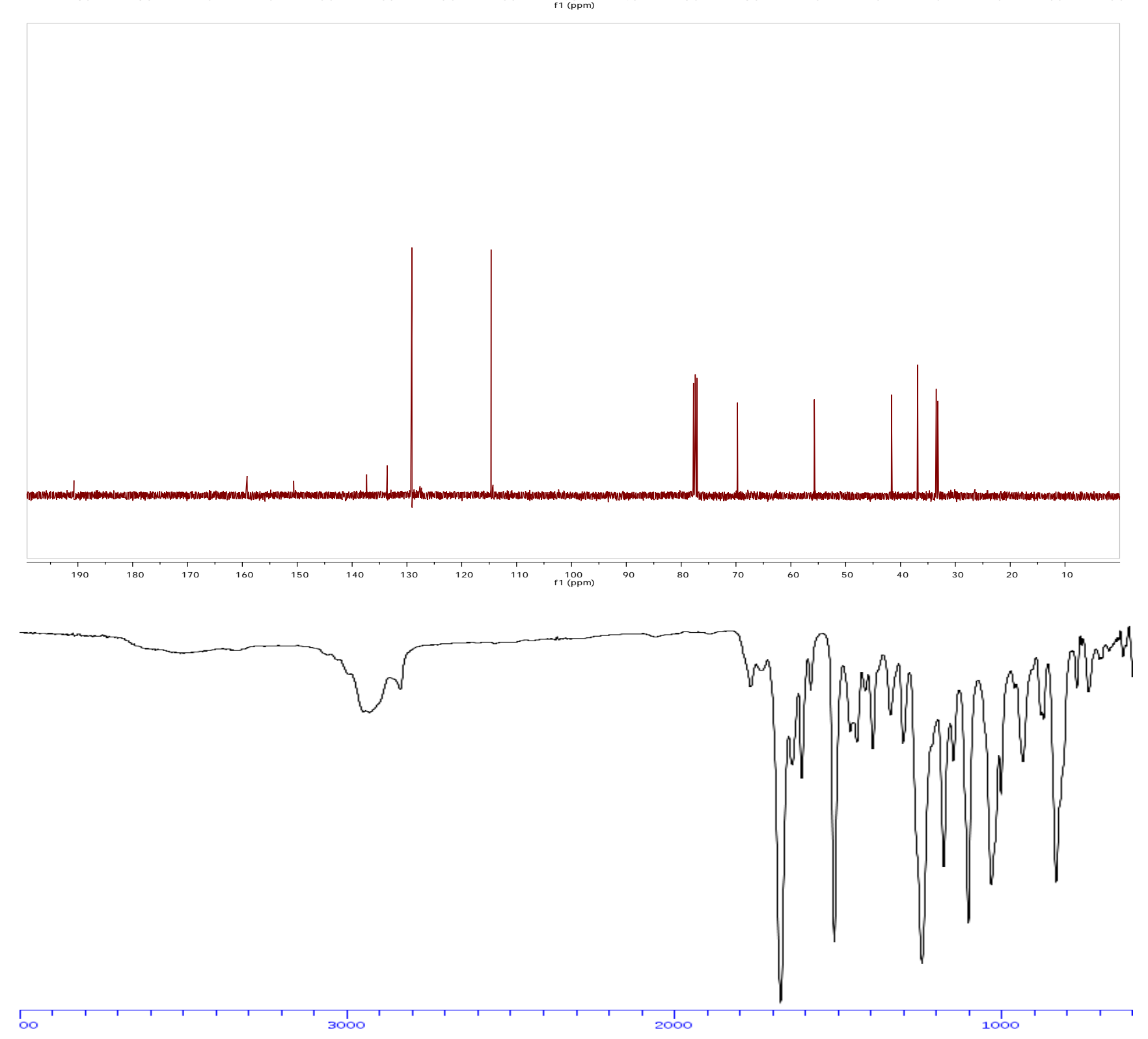

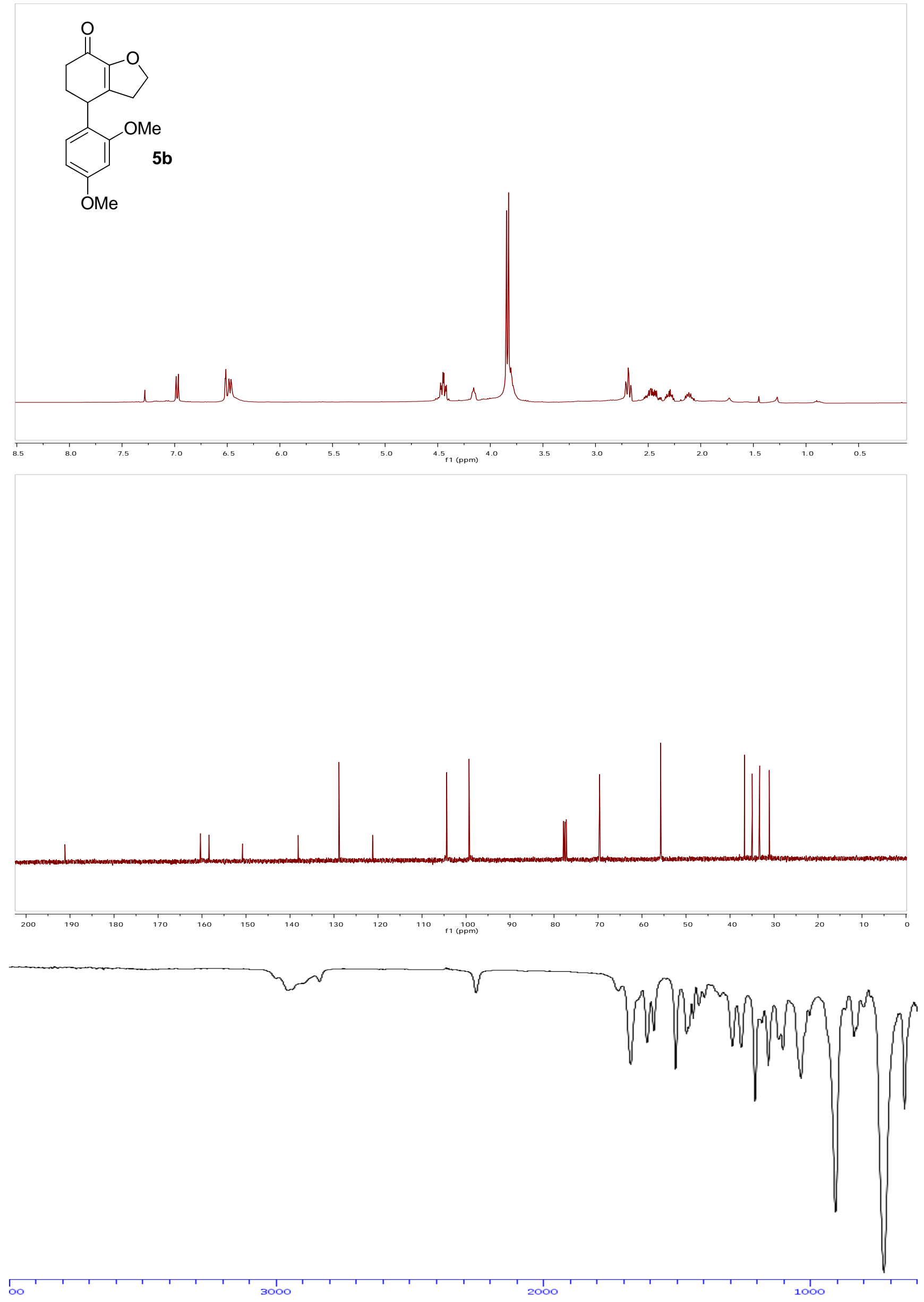
<smiles>COc1ccc(C2CC2C(=O)c2cc3ccccc3n2C)cc1</smiles>
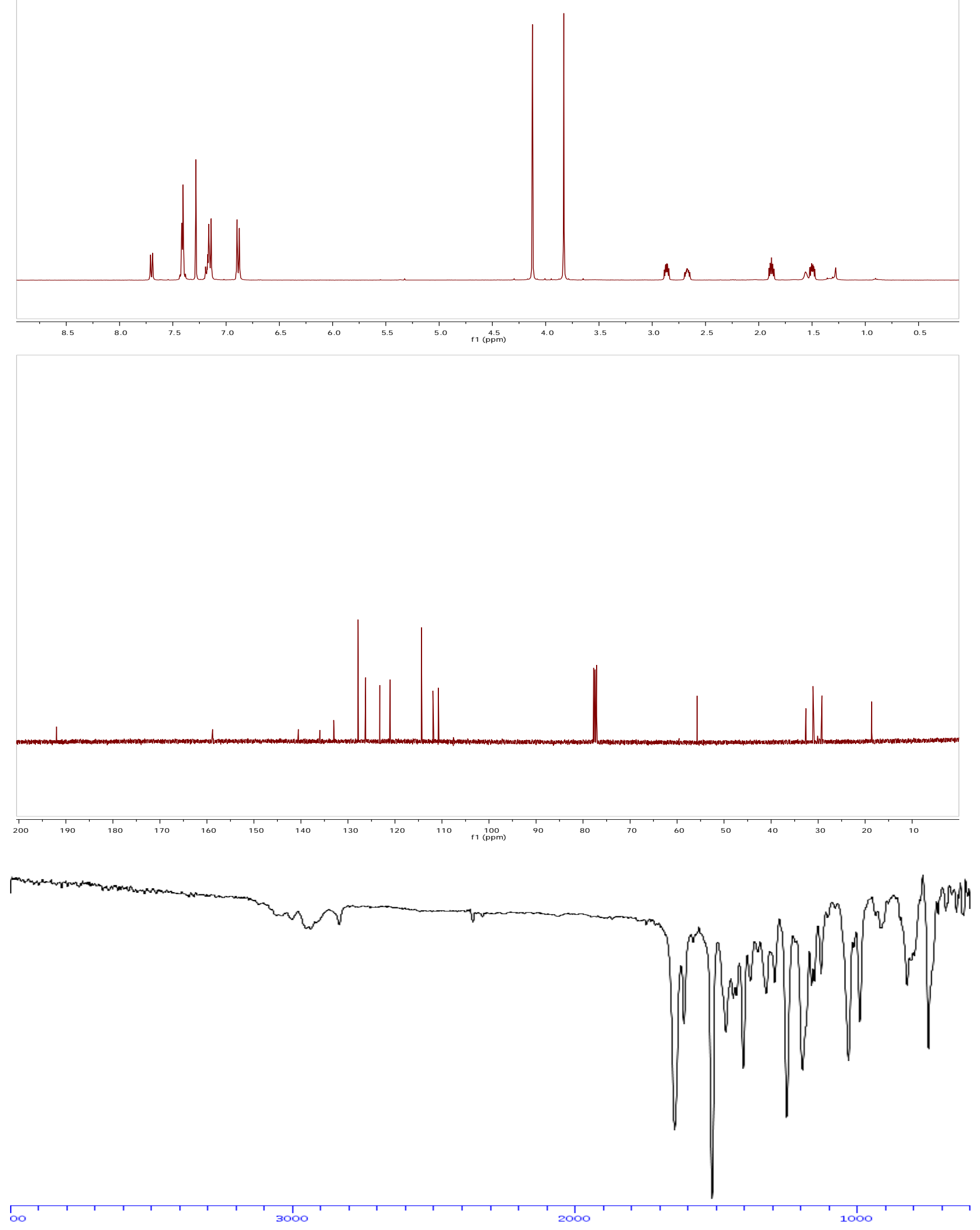

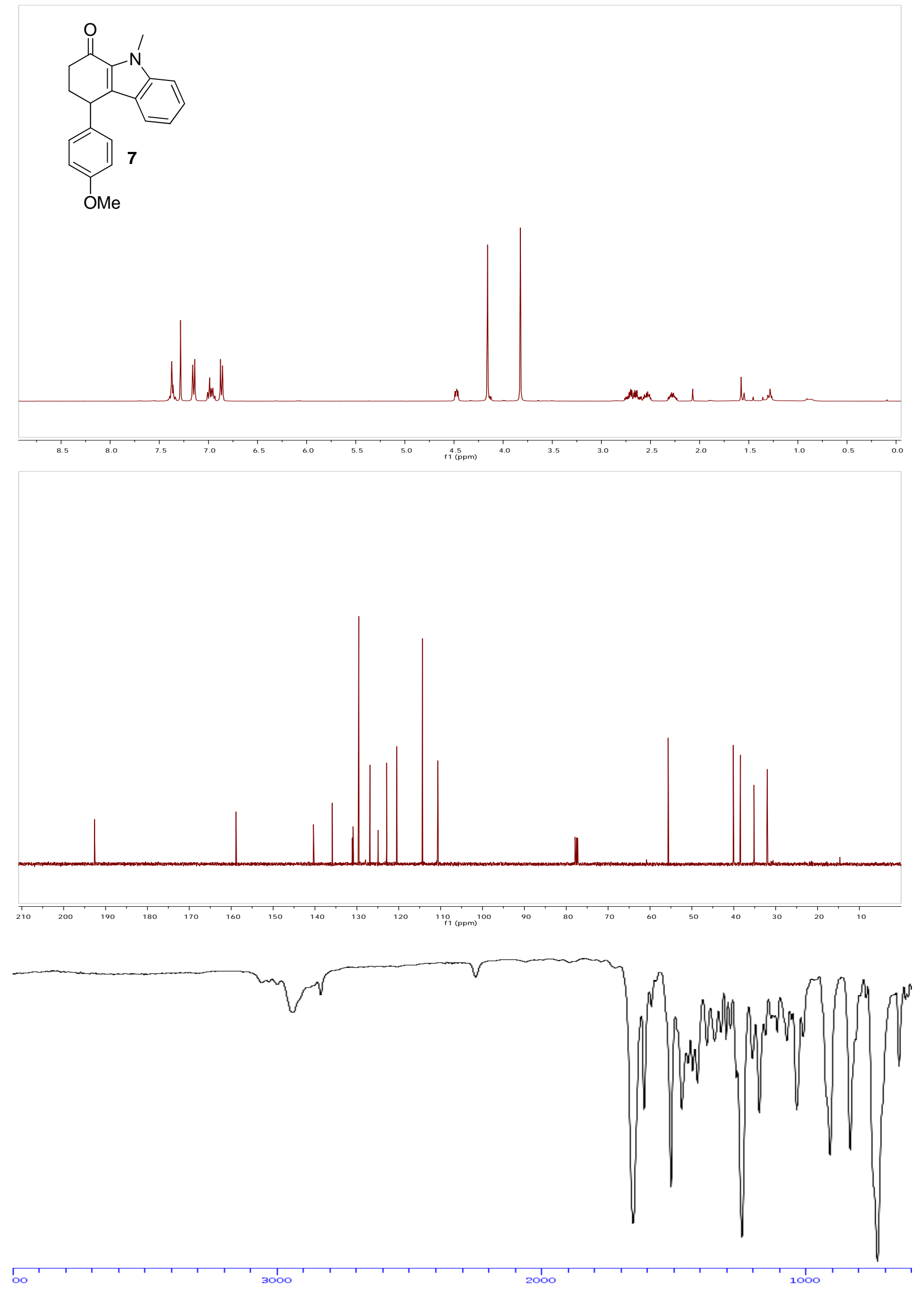
orts

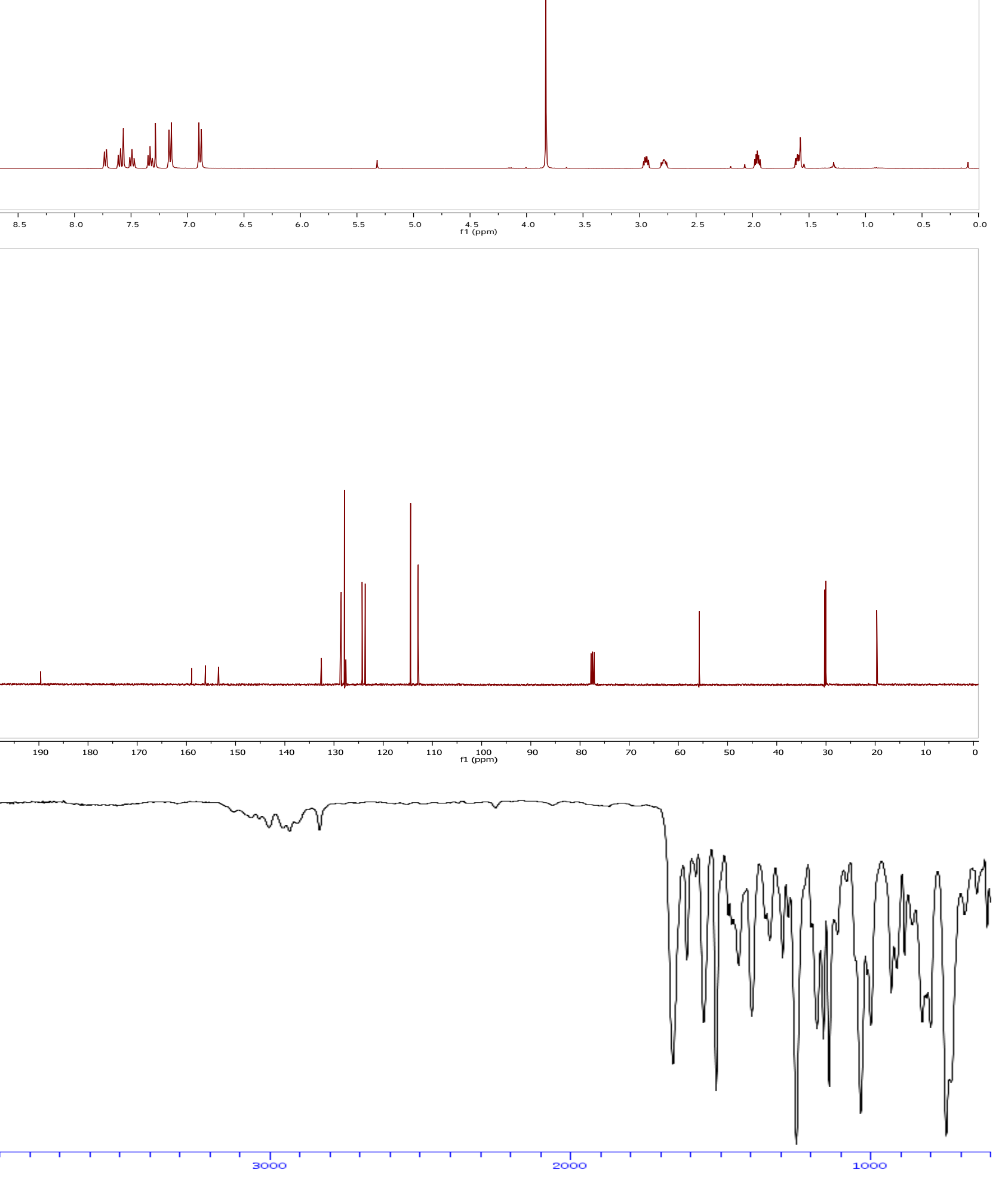



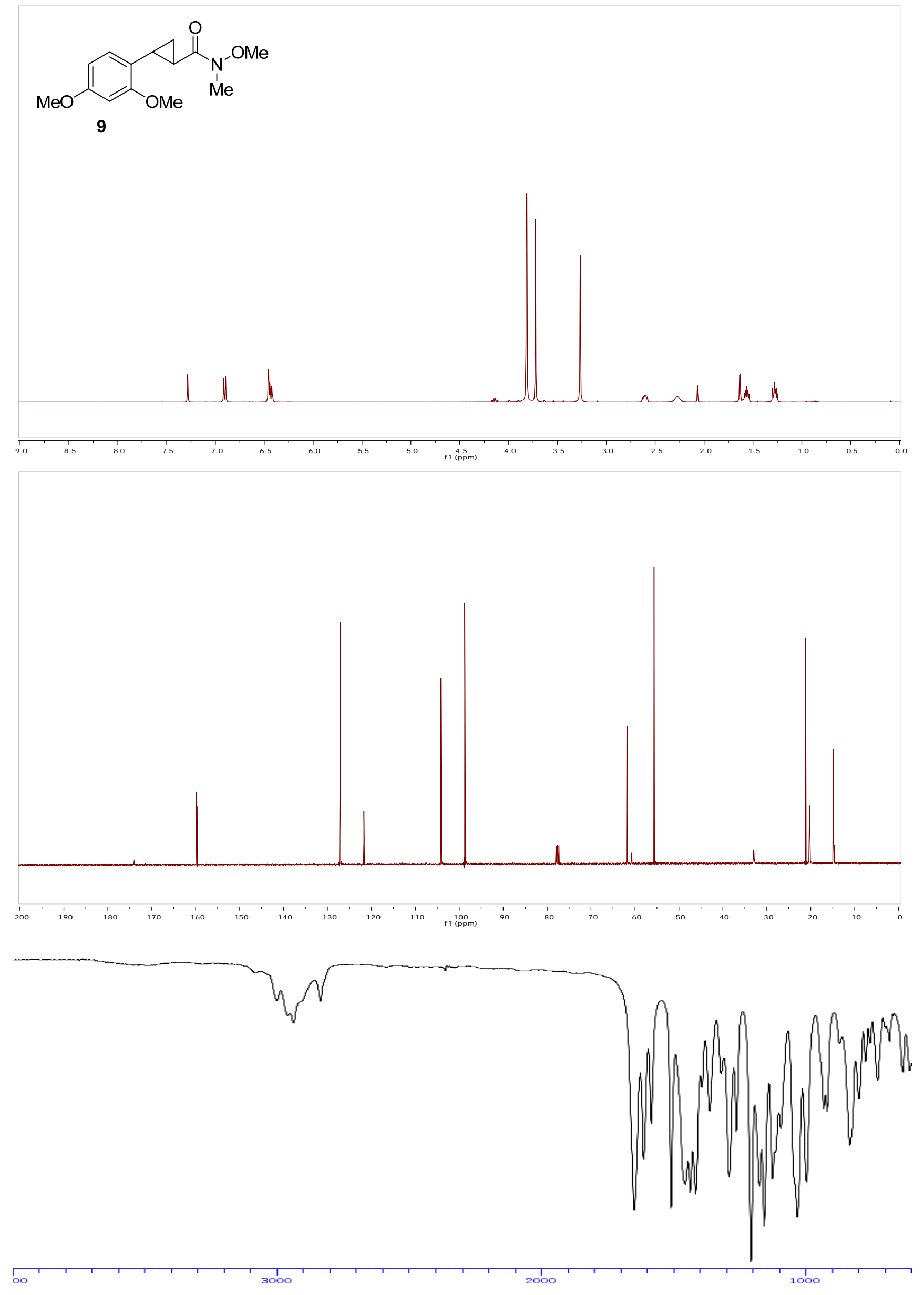

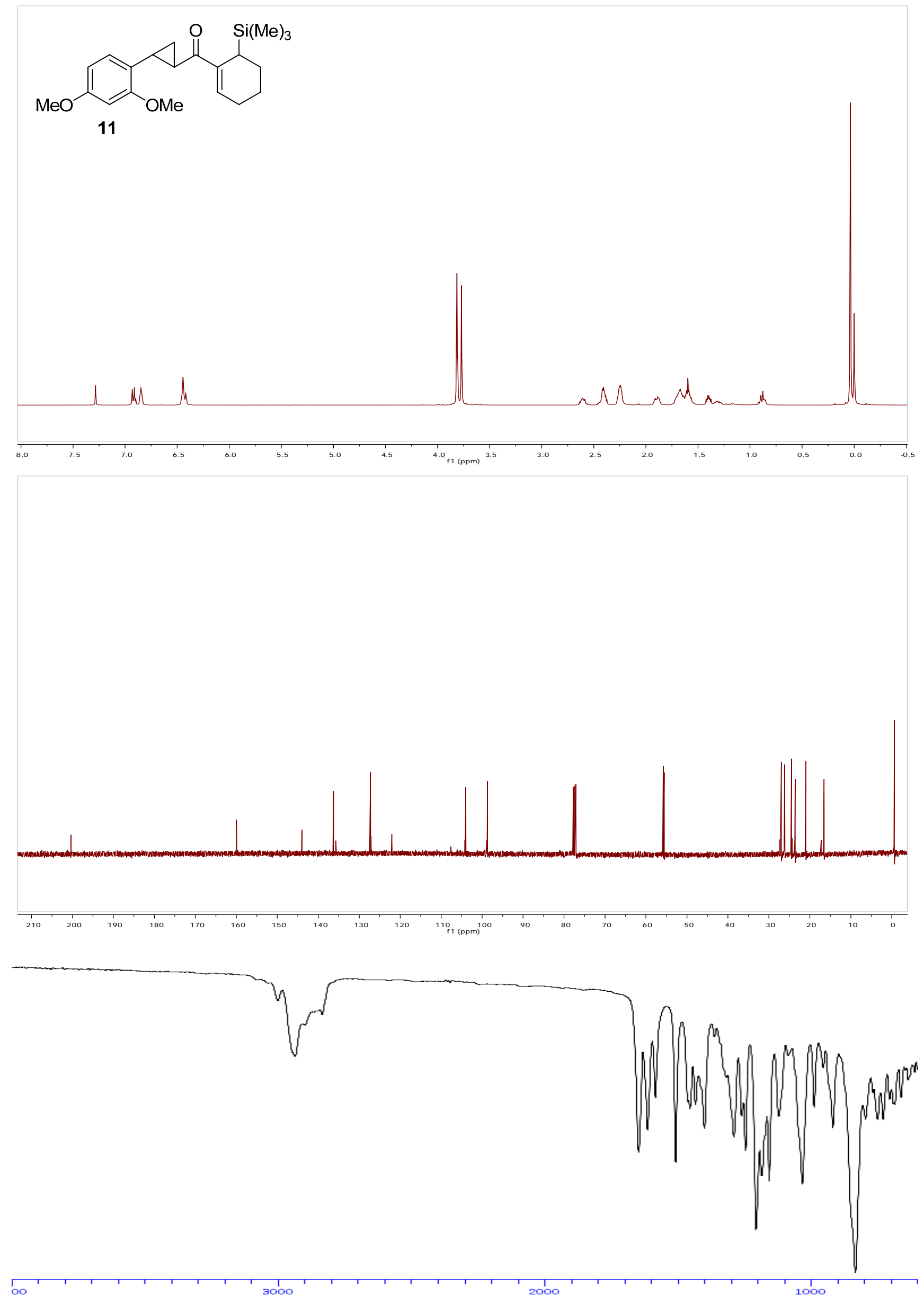

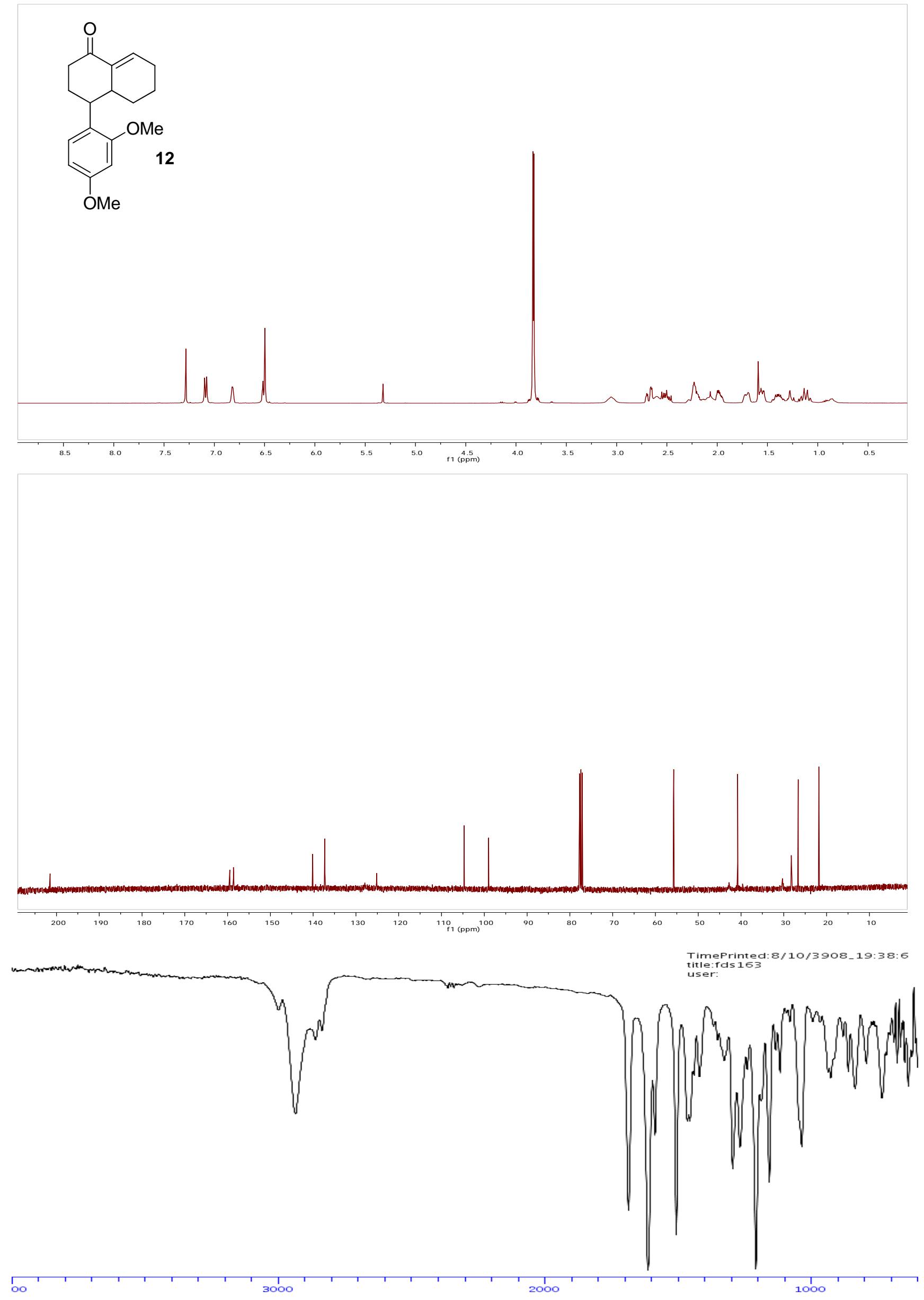

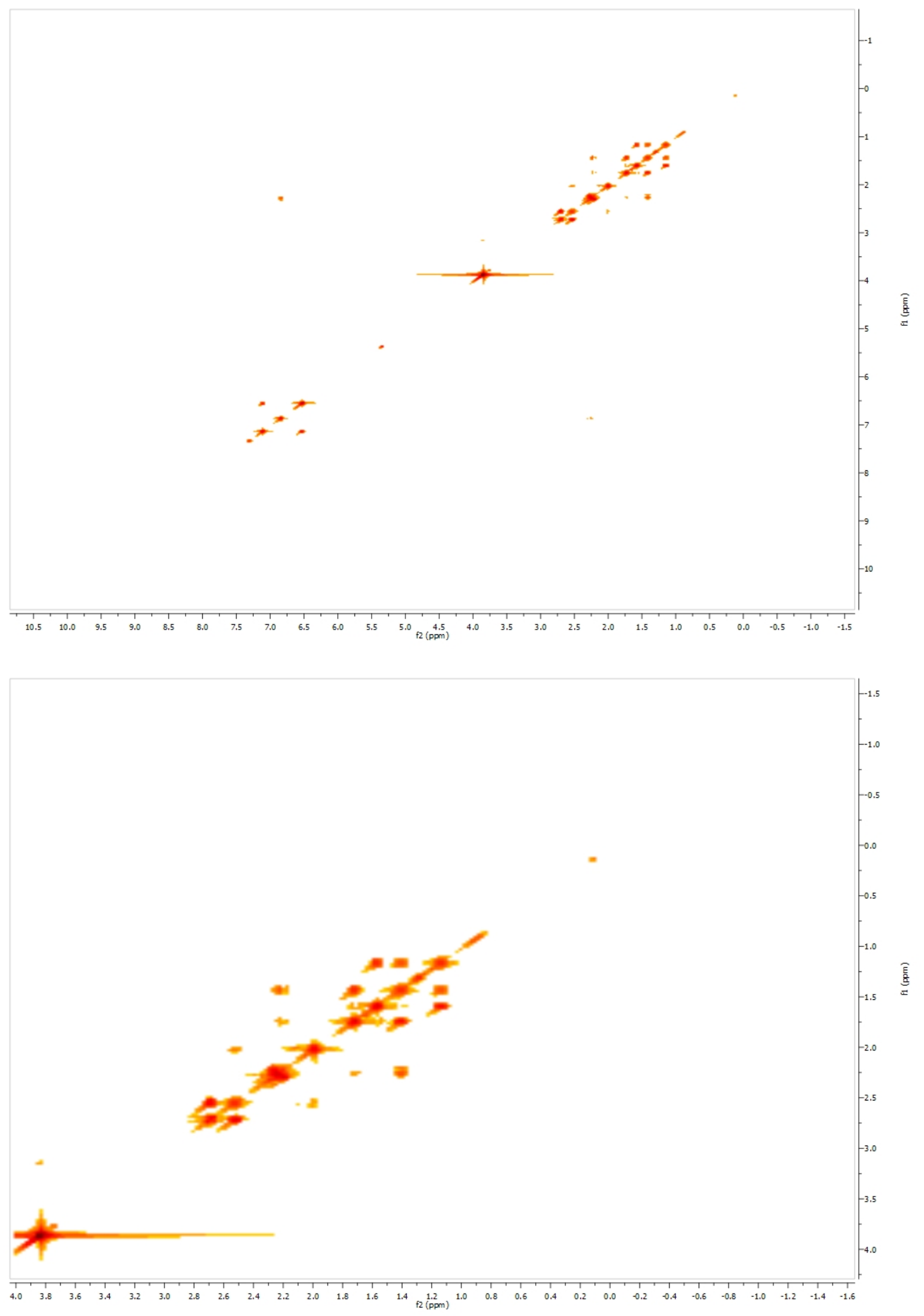

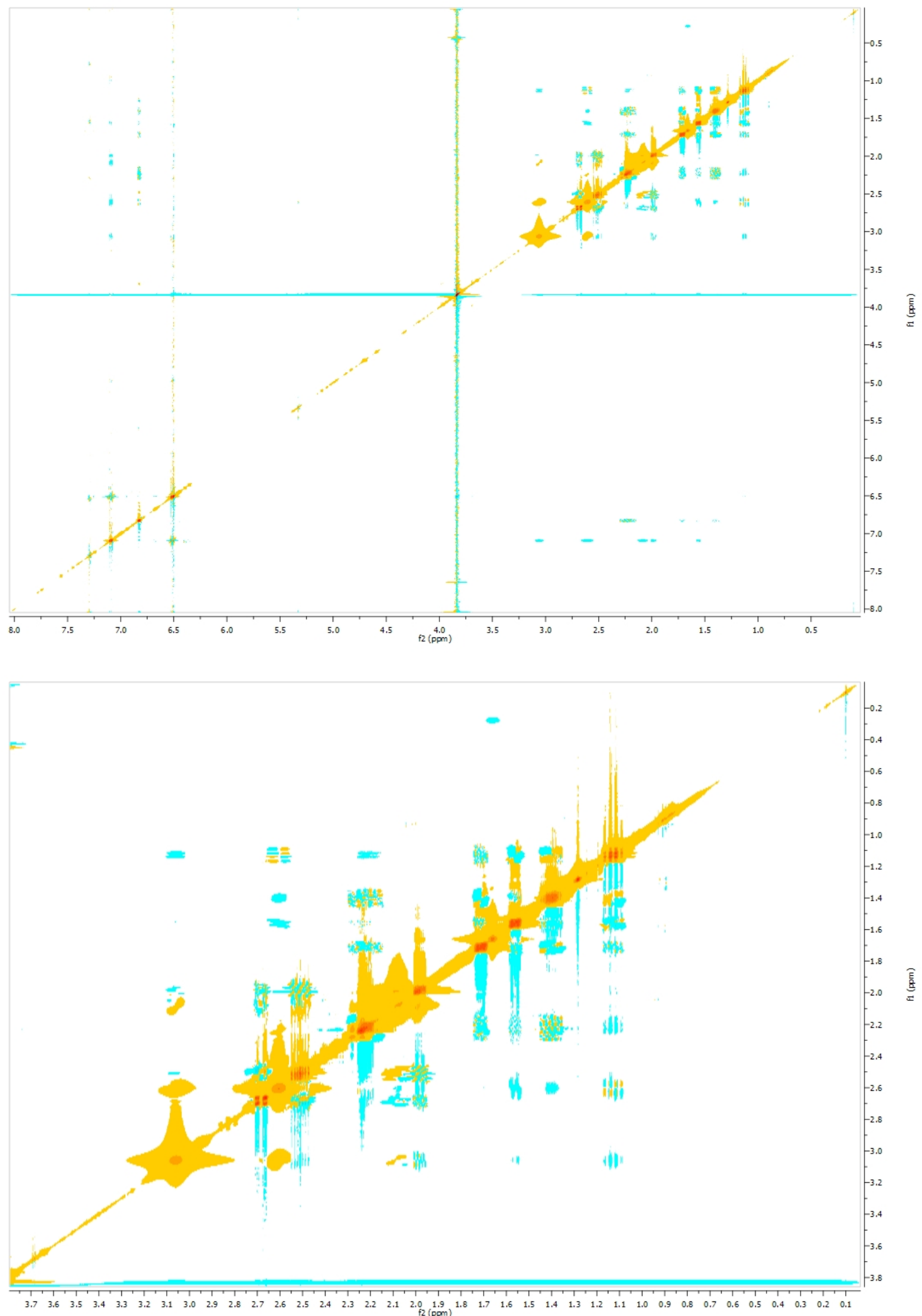

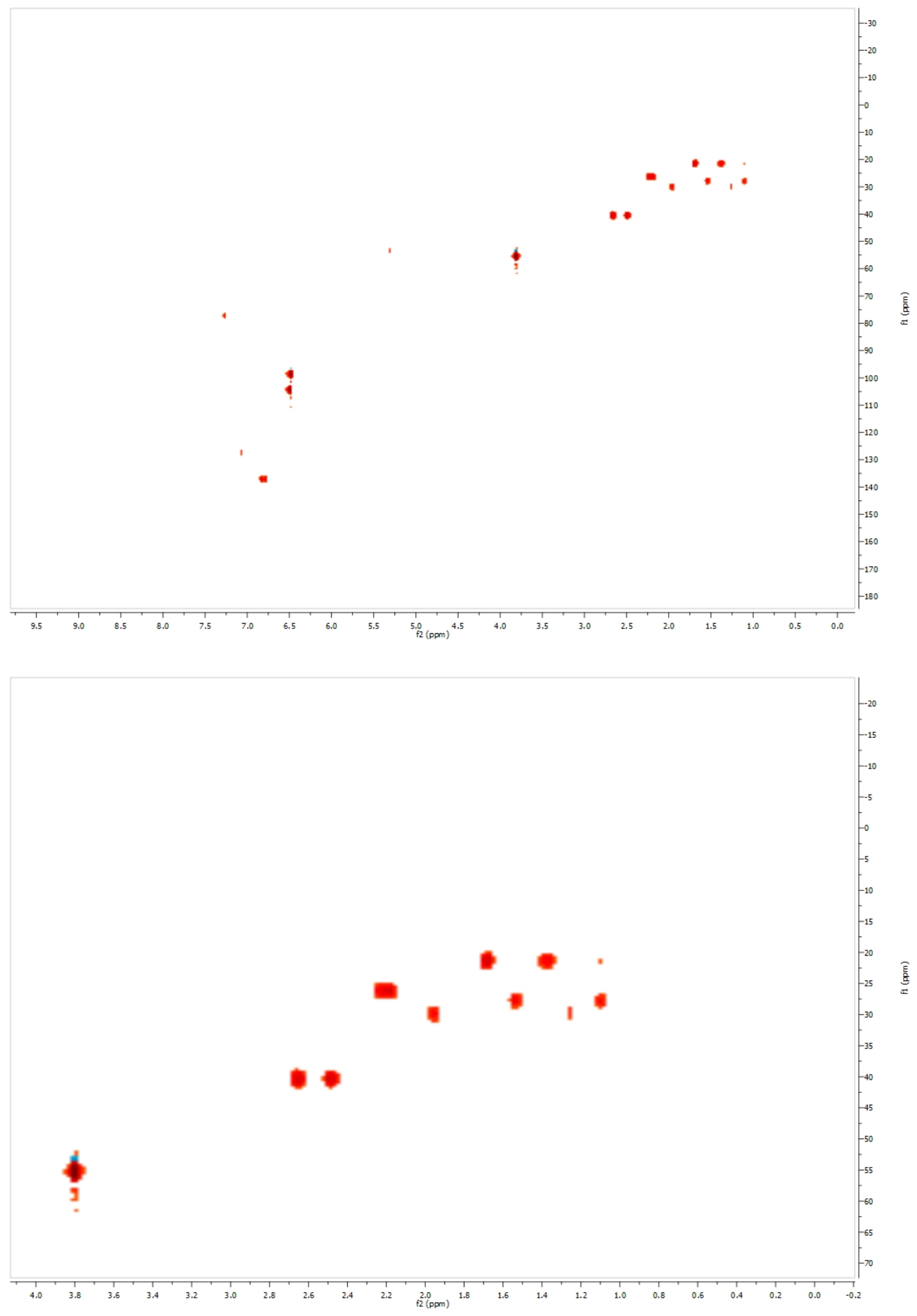
<smiles>COc1ccc(C2CC2C(=O)C2=CCCCC2)cc1</smiles>

15
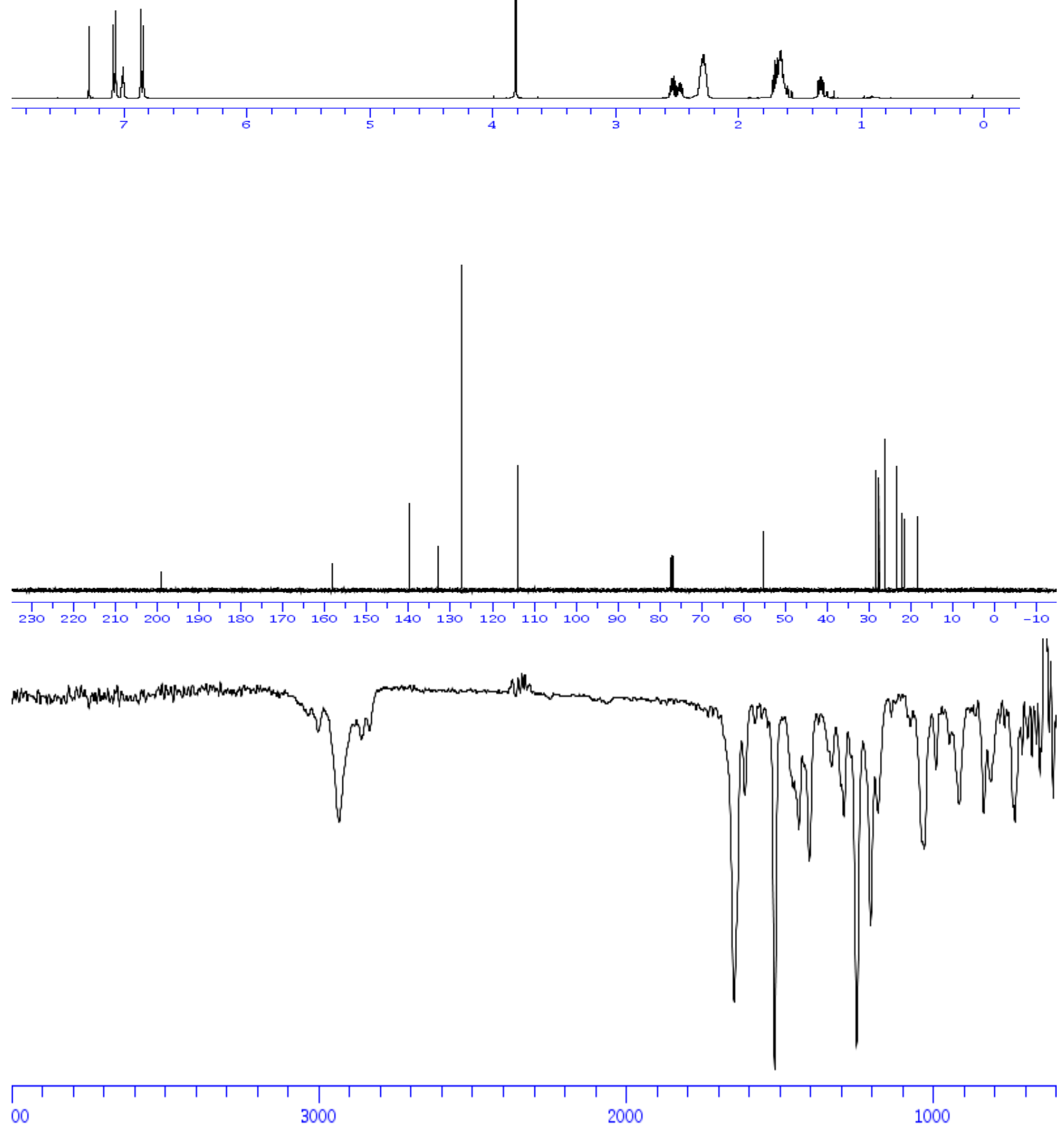
<smiles>COc1ccc(C(O)CCC(=O)C2=CCCCC2)cc1</smiles>

16
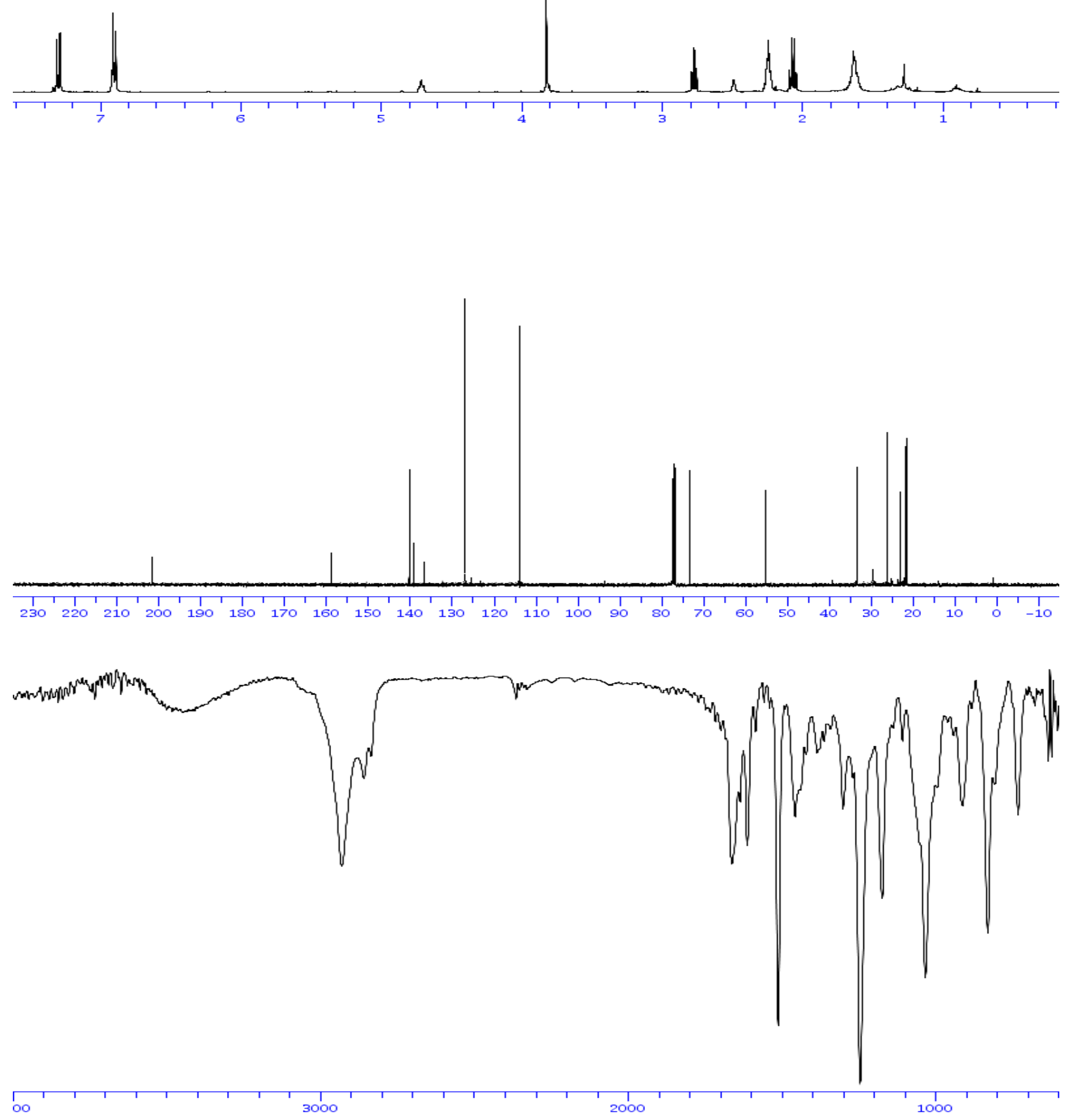
<smiles>COc1ccc(/C=C/C(=O)C2=CCCCO2)cc1</smiles>
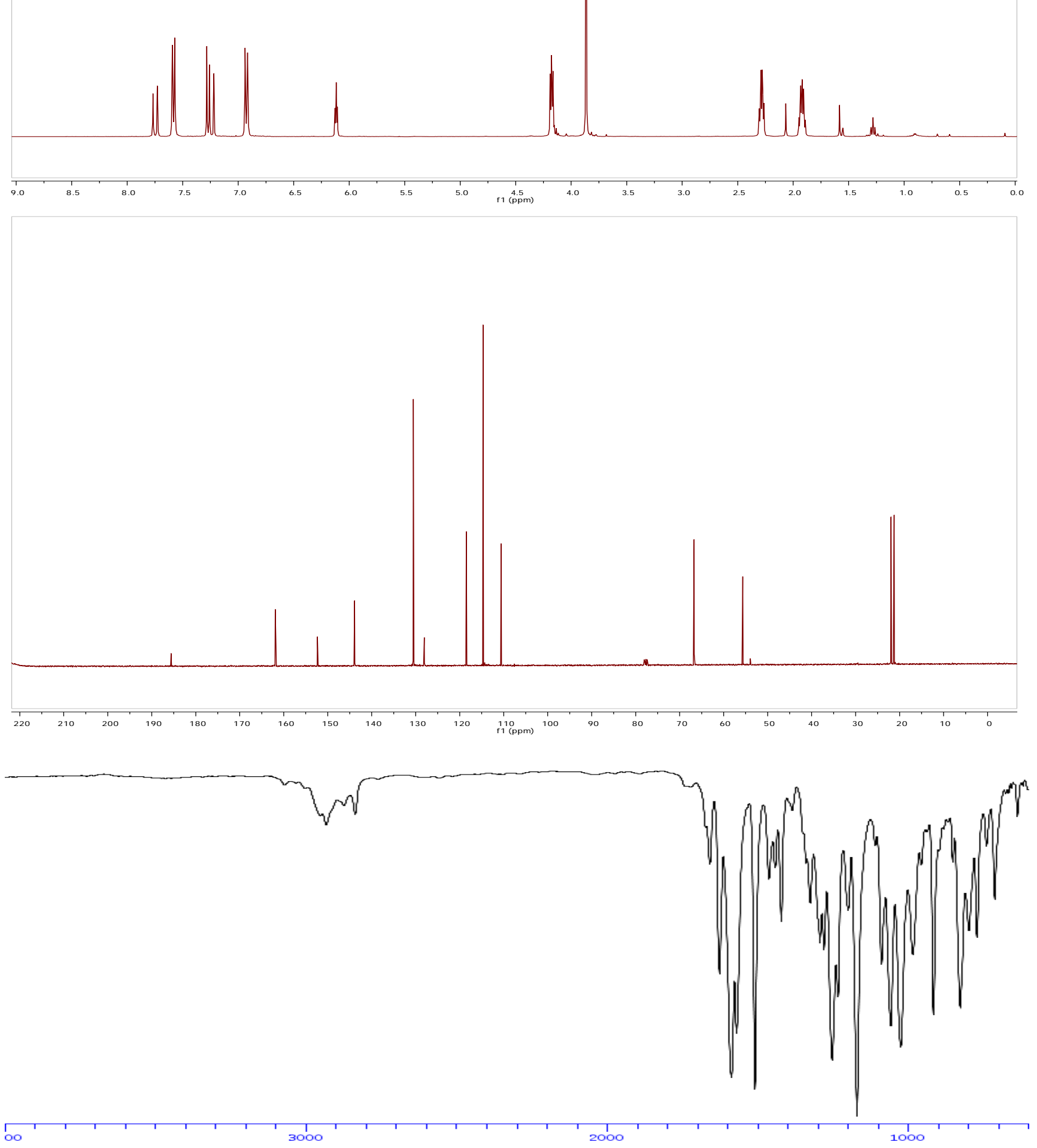

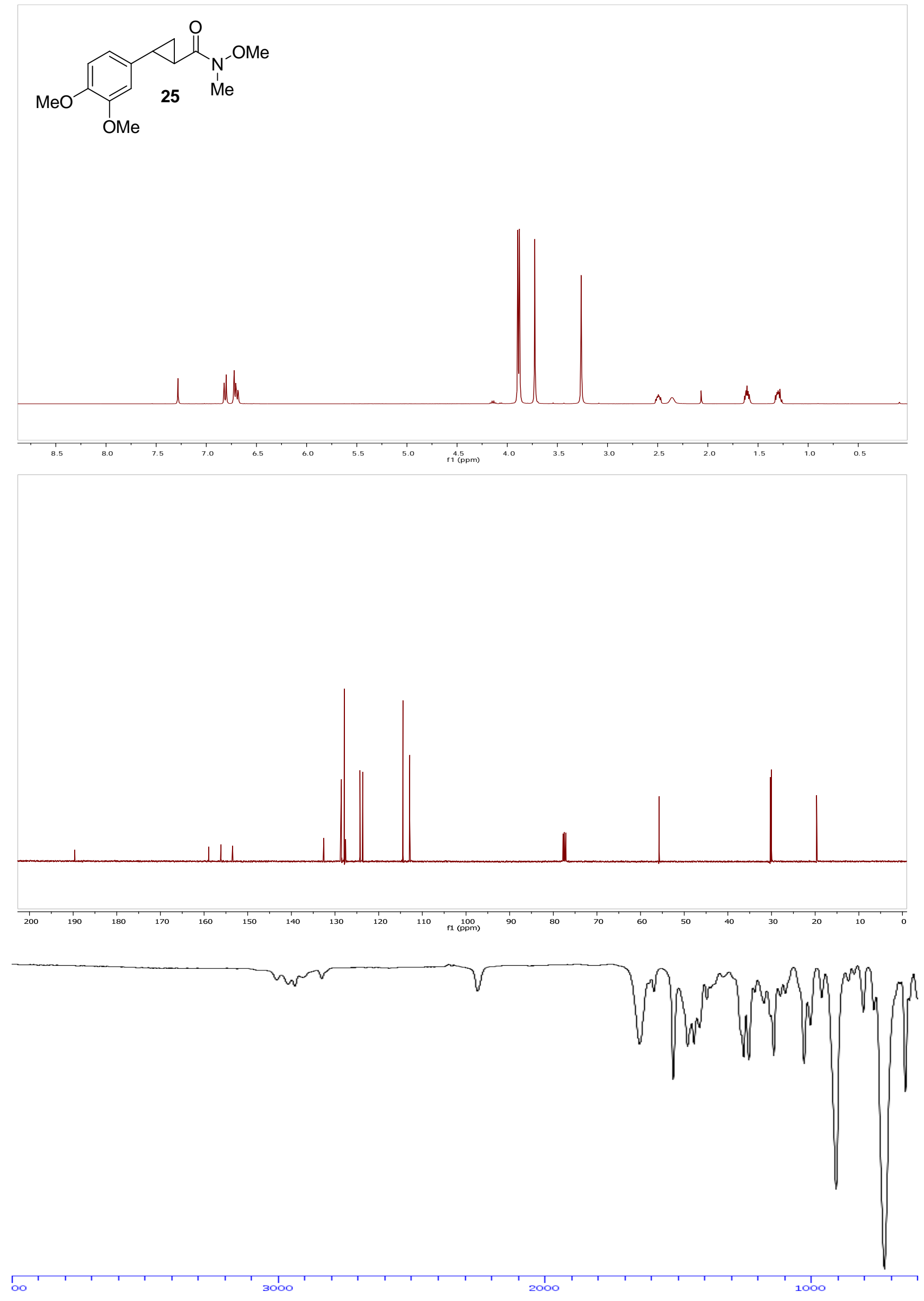

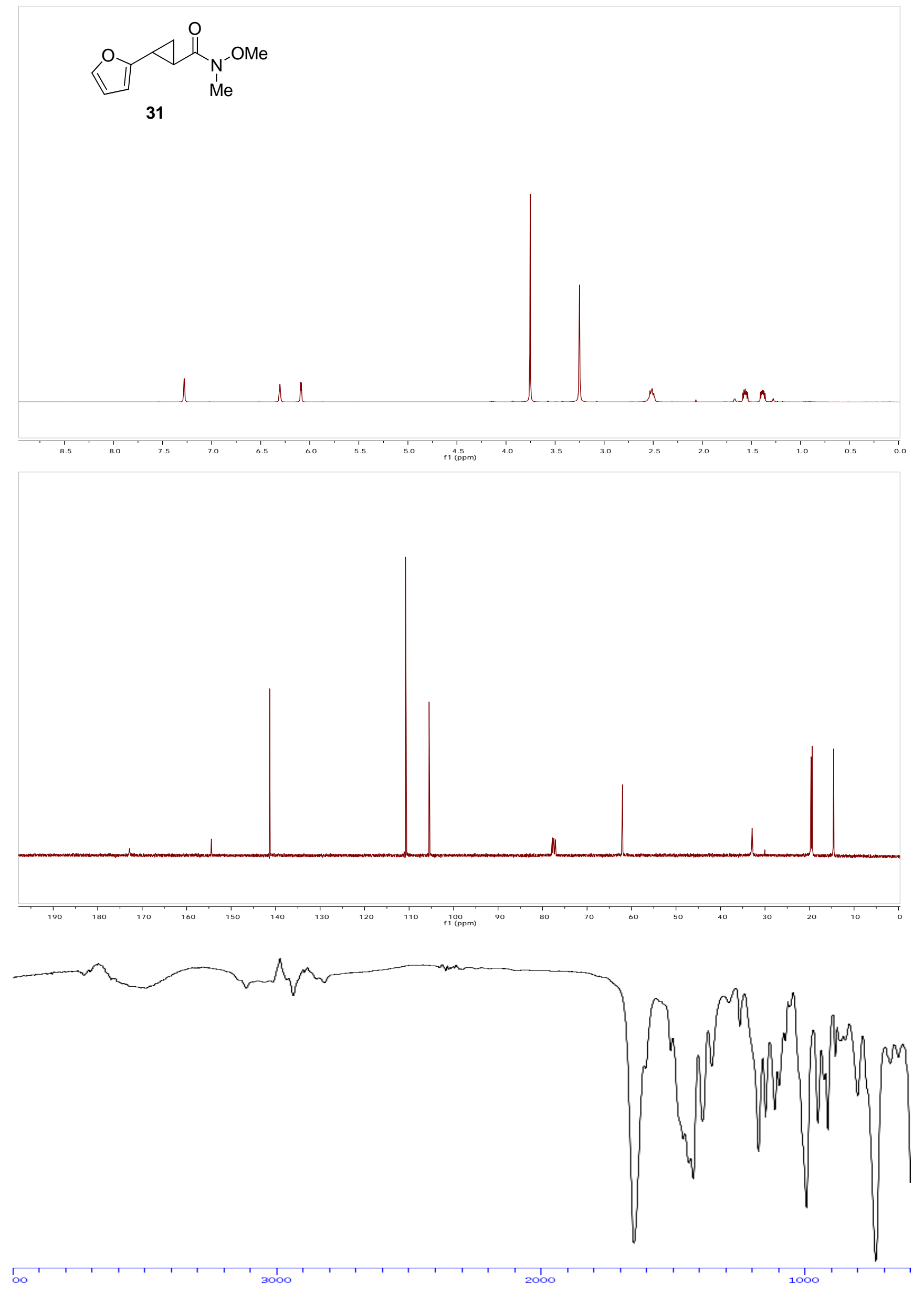

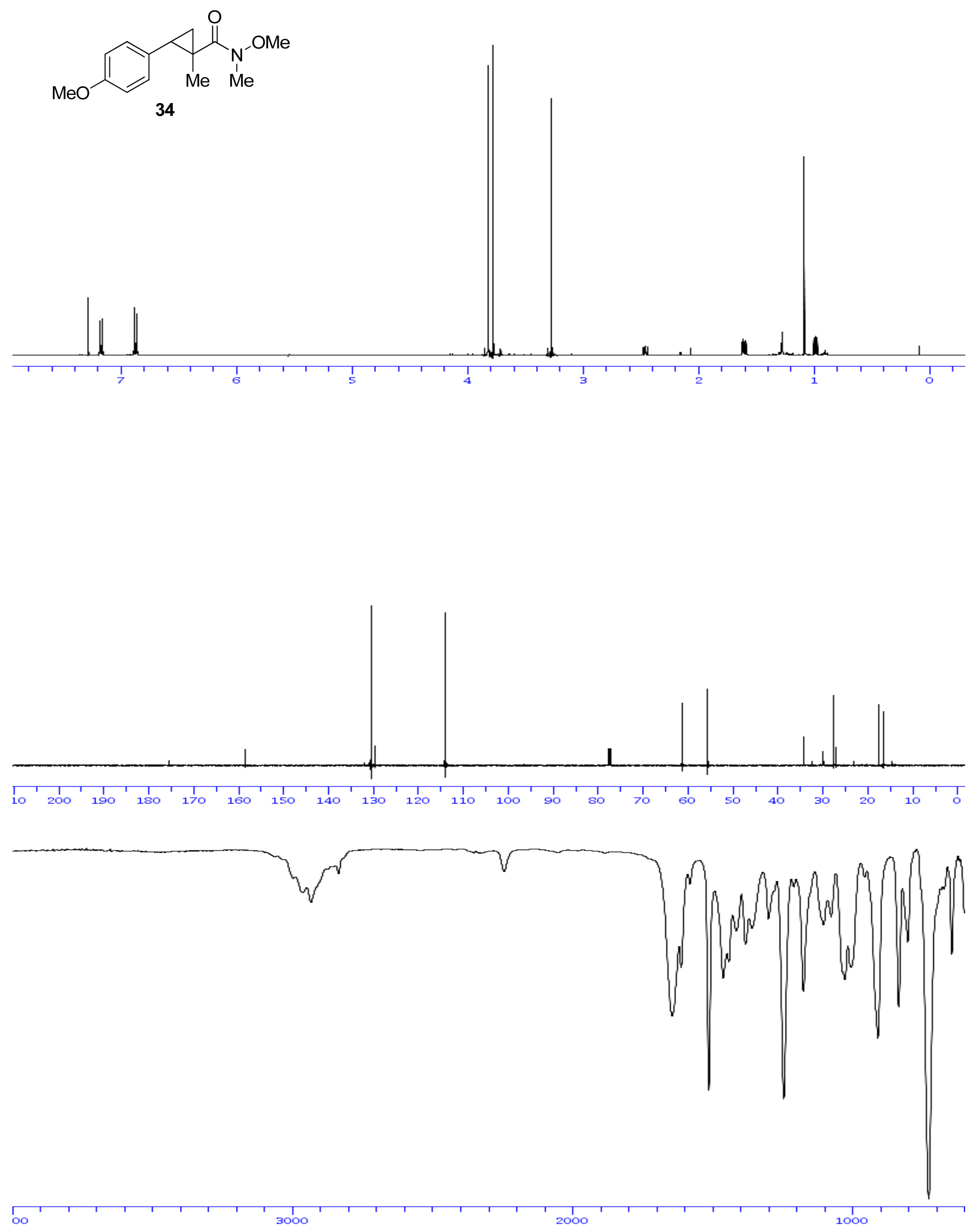
of

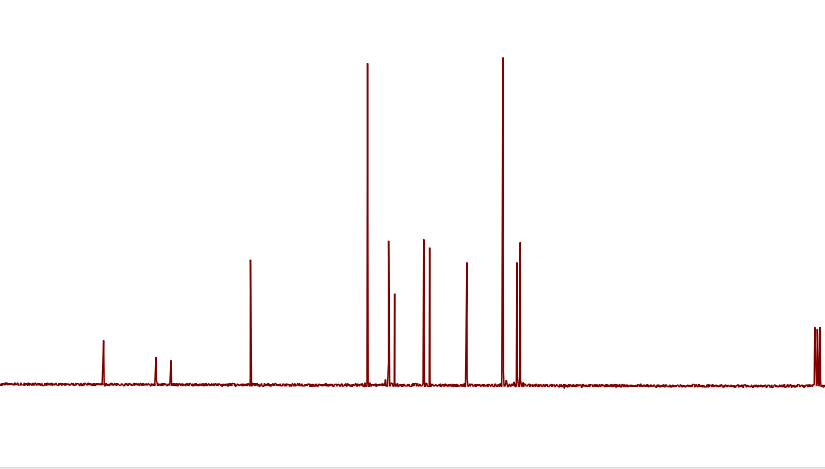

